\title{
LOSS OF UBIQUITIN LIGASE STUB1 AMPLIFIES IFNy-R1/JAK1 SIGNALING AND SENSITIZES TUMORS TO IFNY
}

Georgi Apriamashvili1,5, David W. Vredevoogd ${ }^{1,5}$, Oscar Krijgsman ${ }^{1,6}$, Onno B. Bleijerveld ${ }^{2,4,6}$, Maarten A. Ligtenberg ${ }^{1}$, Beaunelle de Bruijn ${ }^{1}$, Julia Boshuizen ${ }^{1}$, Daniela D’Empaire Altimari', Nils L. Visser ${ }^{1}$, James D. Londino ${ }^{3}$, Maarten Altelaar ${ }^{2,4}$, Daniel S. Peeper ${ }^{*}$

1Division of Molecular Oncology and Immunology, Oncode Institute, The Netherlands Cancer Institute, Plesmanlaan 121, 1066 CX Amsterdam, The Netherlands 2Proteomics Core Facility, The Netherlands Cancer Institute, Plesmanlaan 121, 1066 CX Amsterdam, The Netherlands

3Division of Pulmonary, Critical Care and Sleep Medicine, The Ohio State University 3 Wexner Medical Center, 410W $10^{\text {th }}$ Avenue, Columbus, Ohio, USA

$4{ }^{4}$ Biomolecular Mass Spectrometry and Proteomics, Bijvoet Center for Biomolecular 5 Research and Utrecht Institute for Pharmaceutical Sciences, University of Utrecht, and Netherlands Proteomics Center, Padualaan 8, $3584 \mathrm{CH}$ Utrecht, The Netherlands ${ }^{5}$ These authors contributed equally

$18{ }^{6}$ These authors contributed equally

"Correspondence to:

2 Daniel S. Peeper

3 Oncode Institute

4 Netherlands Cancer Institute

25 Plesmanlaan 121

$61066 \mathrm{CX}$, Amsterdam

27 The Netherlands

8 E-mail: d.peeper@nki.nl

Tel.: +31 205122002 


\section{Abstract}

34 Despite the success of immune checkpoint blockade (ICB) most patients fail to 35 respond durably, in part owing to reduced interferon gamma (IFNy) sensitivity. Thus, 36 elevating tumor IFNy-receptor 1 (IFNy-R1) expression to enhance IFNy-mediated 37 cytotoxicity is of potential clinical interest. Here, we show that increased IFNy-R1 38 expression sensitizes tumors to IFNy-mediated killing. To unveil the largely undefined 39 mechanism governing IFNY-R1 expression, we performed a genome-wide 40 CRISPR/Cas9 screen for suppressors of its cell surface abundance. We uncovered 41 STUB1 as key mediator of proteasomal degradation of the IFNY-R1/JAK1 complex. 42 STUB1 inactivation amplified IFNy signaling, thereby sensitizing to cytotoxic T cells, 43 but also inducing PD-L1. STUB1 loss in a rational combination with PD-1 blockade 44 strongly inhibited melanomas in vivo. Clinically corroborating these results, a STUB1$45 \mathrm{KO}$ gene signature was strongly associated with anti-PD-1 response. These results 46 uncover STUB1 as pivotal regulator of IFNy tumor signaling and provide a rationale 47 for its inhibition combined with anti-PD-1. 


\section{Introduction}

Although immune checkpoint blockade (ICB) has been a major clinical success in the treatment of a variety of cancer indications, the majority of patients fail to show durable clinical responses ${ }^{1,2}$. This is caused by both upfront and acquired resistance mechanisms $^{3-7}$, for which predictive biomarkers are being actively sought ${ }^{8-17}$. A common resistance mechanism relates to the insensitivity that tumors develop against cytokines secreted by cytotoxic $\mathrm{T}$ cells, including IFNy and $\mathrm{TNF}^{4,5,18,19}$. IFNy can promote antitumor activity indirectly, by inducing secretion of lymphocyte-attracting chemokines such as CXCL9, CXCL10 and CXCL11 and by skewing the attracted immune infiltrate to be more inflammatory. Conversely, IFNy can inhibit tumorigenesis directly, by improving antigen processing and presentation, and by inducing the expression of cell cycle inhibitors, such as p21 ${ }^{\text {Cip1 }}$, and pro-apoptotic proteins, such as caspase 1 and caspase $8^{20,21}$. Moreover, IFNy can sensitize tumor cells to other $T$ cell-derived effector cytokines by increasing the expression of FAS and TRAIL receptors $^{22,23}$.

In line with these biological functions, expression of IFNy response genes in tumors is associated with better responses to immunotherapy ${ }^{17,24}$. These clinical findings are underscored by preclinical research showing a critical role for IFNy in hindering tumorigenesis and maintaining tumor control ${ }^{25}$. Conversely, aberrations in the IFNy response pathway, such as inactivation of JAK1, are associated with resistance to immunotherapy ${ }^{4,5,18}$.

Although the IFNy signaling pathway has been studied extensively, and different regulatory mechanisms of this pathway have been uncovered, less is known about the cell-autonomous regulation of the IFNy receptor 1 (IFNy-R1), the essential ligandbinding receptor chain for IFNy. Multiple experimental and clinical approaches have identified that tumor cells benefit from either loss or reduction in IFNY-R1 levels in the context of ICB therapy ${ }^{5}$ or T cell pressure ${ }^{6,26,27}$.

However, to our knowledge the converse has not been studied. Specifically, the possibility that tumor cells with high (or induced) IFNy-R1 expression show increased sensitivity to IFNy-induced cytotoxicity has remained untested. Whereas disruption of IFNy signaling is an established cancer trait contributing to immune escape, a scenario 
83 in which increased IFNy signaling would lead to increased T cell sensitivity may be of 84 clinical interest. This is the first question we addressed in this study. The answer 85 prompted a second one, namely, which mechanisms govern the expression of IFNy86 R1. To address this, we performed a genome-wide CRISPR/Cas9 knockout screen. 87 Lastly, we translated our findings to a preclinical setting, demonstrating their 88 therapeutic and clinical relevance. 


\section{Results}

\section{High IFNy-R1 expression results in increased sensitivity of tumor cells to $\mathrm{T}$ cell} killing

Whereas it is established that loss of the IFNy-R1 ablates IFNy tumor signaling ${ }^{5,25}$, it is unknown whether the converse is also true. To assess whether increased abundance of IFNy-R1 augments the susceptibility of tumor cells to cytotoxic T cells, we took advantage of the heterogeneity we observed for its expression levels in the human melanoma cell line D10. We FACsorted tumor cells with high and low expression levels of IFNY-R1 (Fig. 1a and b). As a control protein, we determined the expression of another cell surface protein, PD-L1, which was expressed identically in the IFNY-R1 $1^{\text {High }}$ and IFNy-R1 $1^{\text {Low }}$ cell populations (Fig. 1c). We then investigated whether IFNY-R1 $1^{\text {High }}$ and IFNY-R1 $1^{\text {Low }}$ cells differentially responded to IFNY. By flow cytometry, we observed that IFNY-R1 ${ }^{\text {High }}$ cells induced PD-L1 to a greater extent upon IFNy treatment than IFNy-R $1^{\text {Low }}$ cells. This result indicates that the expression levels of the endogenous IFNY-R1 protein dictate the strength of the response to IFNY (Fig. 1c). This effect had also a biological consequence: in a competition experiment, IFNy treatment was two-fold more toxic to IFNY-R1'High than to IFNY-R1 ${ }^{\text {Low }}$ cells (Supplementary Fig. 1a and b).

We repeated this experiment with cytotoxic $T$ cells, employing the matched tumor HLA-A*02:01+/MART1 ${ }^{+}$and 1D3 TCR T cell system we previously developed ${ }^{19}$. In this experiment also, IFNy-R1 ${ }^{\text {High }}$ melanoma cells showed higher susceptibility to $\mathrm{T}$ cell killing than IFNY-R1 ${ }^{\text {Low }}$ cells (Fig. 1d, e). Thus, the expression level of IFNY-R1 is heterogeneous even in an established tumor cell line. More importantly, these results demonstrate that this variation has a biological consequence, in that higher IFNY-R1 expression results in increased sensitivity of tumor cells to $\mathrm{T}$ cell killing.

\section{Whole genome CRISPR/Cas9 screen identifies regulators of IFNY-R1 expression}

Because this observation could have potential therapeutic relevance, it was important to first dissect the mechanism governing IFNY-R1 expression in an unbiased fashion. To identify novel regulators of cell surface-expressed IFNY-R1, we performed a CRISPR/Cas9 knockout screen (Fig. 1f). Cas9-expressing human D10 melanoma cells were lentivirally transduced with a genome-wide knockout library ${ }^{28}$, in duplicate. After two days of puromycin selection, we harvested a library reference sample. After 
an additional eight days of culturing, we FACsorted both the lower (IFNy-R1 ${ }^{\text {Low }}$ ) and upper (IFNy-R1 High) $10 \%$ of IFNy-R1-expressing cell populations (as well as an unsorted bulk reference sample, Fig. 1f). Genomic DNA was isolated and sgRNA sequences were amplified by PCR. Analysis of the DNA sequencing data revealed a strong correlation between biological replicates (Supplementary Fig. 1c). By comparing the library reference with unsorted control samples, we confirmed significant depletion of known essential genes ${ }^{29}$ (Supplementary Fig. 1d). These quality control measures illustrate the robustness of the screen.

By MAGeCK analysis ${ }^{30}$, we identified several hits affecting IFNy-R1 expression (Fig. 1g). Comparative analysis of the IFNY-R $1^{\text {High }}$ and IFNY-R1 $1^{\text {Low }}$ melanoma populations revealed that cells carrying sgRNAs targeting IFNGR1 were most abundant in the latter population, again demonstrating the robustness of the screen (Fig. 19). More interestingly, the E3 ubiquitin ligase STIP1 homology and U-box containing protein 1 (STUB1, also known as CHIP and encoded by STUB1) was found as the strongest hit suppressing IFNY-R1 cell surface abundance. We also identified other genes negatively affecting IFNy-R1 expression, including Ancient ubiquitous protein 1 and Uroporphyrinogen Decarboxylase (encoded by AUP1 and UROD, respectively). We performed the same IFNy-R1 regulator screen in a second human melanoma cell line, SK-MEL-23, which was similar in quality (Supplementary Fig. 1e) and also identified STUB1 and UROD (Supplementary Fig. 1f).

To validate these screen hits, we inactivated either STUB1, AUP1 or UROD using two independent sgRNAs for each gene. Whereas cells expressing sgIFNGR1 showed a near-complete loss of IFNY-R1 expression, inactivation of either STUB1 or UROD, and to a lesser extent $A U P 1$, instead resulted in a robust increase of IFNY-R1 abundance (Fig. 1h).

\section{STUB1 specifically regulates the cell surface fraction of IFNy-R1}

152 To determine whether STUB1 functions as a negative regulator of IFNY-R1 expression 153 beyond melanoma, we depleted it by Cas9-mediated knockout from cell lines 154 originating from different tumor indications, and assessed the effect on the expression 155 of IFNY-R1. We again observed strong induction of IFNY-R1 expression in all cell lines 
tested, indicating that STUB1 has a key role in limiting IFNY-R1 expression across different tumor types (Fig. 2a and b).

This broad effect prompted us to mechanistically dissect how STUB1 regulates IFNYR1 expression. qPCR analysis for IFNGR1 showed that its transcript levels were indistinguishable between WT and STUB1-deficient cells (Supplementary Fig. 2a). Therefore, we focused our attention on a post-transcriptional mode of regulation. We first determined in which cellular compartment STUB1 regulates IFNY-R1 expression. Cell lysates of STUB1-proficient and STUB1-deficient cells were treated with various deglycosylating enzymes. The strongest increase in IFNY-R1 was seen in the high molecular weight, Endo-H-resistant species of IFNy-R1. This suggests that the regulation of IFNY-R1 by STUB1 occurs after it passes through the endoplasmic reticulum (Supplementary Fig. 2b, c).

IFNY-R1 manifested as multiple protein species that were distinguishable by SDSPAGE analysis (Supplementary Fig. 2b). To determine which of these forms are located at the tumor cell surface, we performed biotin labeling and immunoprecipitation of cell-surface proteins ${ }^{31}$. This analysis showed that solely the high molecular weight, Endo-H-resistant, species of IFNY-R1 resides at the plasma membrane (Supplementary Fig. 2d). This result implies that STUB1 specifically regulates the cell surface fraction of IFNY-R1, which is in accordance with our flow cytometry findings.

STUB1 destabilizes IFNY-R1 in JAK1-dependent and JAK1-independent manners

181 STUB1, initially identified as a co-chaperone ${ }^{32}$, acts as an E3 ubiquitin ligase ${ }^{33,34}$ that affects protein stability by mediating proteasomal degradation ${ }^{34-36}$. Therefore, and in accordance with our observation that STUB1 loss does not affect IFNGR1 mRNA levels, we hypothesized that it destabilizes the IFNY-R1 protein. To test this, we profiled the proteomes of cells expressing either a non-targeting control sgRNA (sgCtrl) or a STUB1-targeting sgRNA (sgSTUB1) by mass spectrometry. This analysis not only confirmed our observation that STUB1 inactivation increases IFNY-R1 levels, 188 but it also revealed a marked increase in the abundance of the JAK1 protein (Fig. 2c). 189 This finding was confirmed by the same analysis in a second cell line (Supplementary 
190 Fig. 2e). It was also validated by immunoblotting for IFNY-R1 and JAK1, in two

191 melanoma cell lines (Fig. 2d, Supplementary Fig. 2f and g). Similar to its regulation

192 of IFNY-R1 expression, STUB1 also affected JAK1 protein stability, as JAK1 transcript

193 levels remained unchanged by STUB1 inactivation (Supplementary Fig. 2h).

195 While it is known that the interaction of IFNY-R1 and JAK1 is essential for the signaling 196 functionality of the IFNy receptor complex ${ }^{37,38}$, a potential role of JAK1 in stabilizing 197 IFNY-R1 levels, and by extension the IFNy receptor complex, has not been reported. 198 We first investigated whether heightened JAK1 expression would suffice to drive 199 increased IFNY-R1 protein stability. Ectopically expressed JAK1 strongly increased 200 IFNy-R1 protein abundance (Fig. 2e), which translated into increased cell surface expression (Fig. 2f), even more so than ectopically expressed IFNGR1 (Fig. 2e-g and Supplementary Fig. $\mathbf{2 i}$ and $\mathbf{j}$ ). This result suggests not only that elevated JAK1 protein levels are sufficient to stabilize IFNY-R1 protein, but also that JAK1 expression may be crucial in dictating the amount of IFNY-R1 present on the cell surface; unexpectedly even more so than IFNGR1 expression itself.

To determine whether elevated JAK1 levels in STUB1-inactivated cells account for the rise in IFNY-R1 abundance, we inactivated JAK1 in a STUB1-deficient background (Fig. $\mathbf{2 h}$ and $\mathbf{i}$ ). This epistasis experiment revealed that STUB1 inactivation still led to an increase in IFNY-R1, albeit to a lesser degree than in the presence of JAK1 (Fig. $2 \mathrm{~h}$ and $\mathbf{i})$. These findings together indicate that STUB1 deficiency promotes IFNY-R1 stabilization both in JAK1-dependent and -independent fashions: STUB1 depletion

213 increases IFNy-R1 levels directly, but also increases JAK1 abundance, which in turn

214 further stabilizes IFNy-R1.

STUB1 drives proteasomal degradation of IFNy receptor complex through IFNy$\mathrm{R} 1^{\mathrm{K} 285}$ and $\mathrm{JAK} 1^{\mathrm{K} 249}$ residues

218 Since STUB1 has been shown to mediate proteasomal degradation of client 219 proteins ${ }^{35,36}$, we next asked whether increased protein levels of IFNY-R1 and JAK1 220 upon STUB1 inactivation were caused by reduced proteasomal degradation. We 221 treated either wildtype or STUB1-deficient cells with MG132, an inhibitor of 222 proteasomal degradation. Western blot analysis of these cell lysates showed a 223 significant induction of IFNy-R1 proteins in wildtype cells upon treatment with MG132 
224 (Fig. 3a-c). In contrast, whereas baseline levels of IFNY-R1 were already increased in

225 STUB1-deficient cells, there was no further induction upon MG132 treatment. A similar

226 observation was made for JAK1 (Fig. 3a-c). These results were recapitulated in an

227 additional cell line (Supplementary Fig. 3a-c).

229 Proteasomal degradation by STUB1 requires its tetracorticopeptide (TPR) domain, 230 which interacts with chaperones such as $\mathrm{HSC} 70^{34-36}$. Therefore, we queried whether

231 this domain is required to destabilize IFNY-R1 and JAK1 protein levels. Reconstitution

232 of full length STUB1 in STUB1-deficient cells resulted in reduction of IFNY-R1 and 233 JAK1 proteins to similar baseline levels as observed in wildtype cells (Fig. 3d,

234 Supplementary Fig. 3d and e). However, STUB1-deficient cells reconstituted with a 235 TPR-domain-deficient isoform retained elevated levels of IFNY-R1 and JAK1 236 comparable to those seen in STUB1-deficient cells (Fig. 3d, Supplementary Fig. 3d 237 and e). Taken together, these results indicate that STUB1 regulates protein turnover 238 of both IFNy-R1 and JAK1 by enabling proteasomal degradation of the latter proteins.

240 To understand in more detail how STUB1 mediates the proteasomal degradation of 241 both factors, particularly which lysine residues are critical targets of STUB1, we 242 queried the changes in the landscape of ubiquitinated proteins upon STUB1 depletion.

243 We immunopurified peptides containing a lysine (K)-epsilon-diglycine motif; a remnant 244 mark of ubiquitinated proteins after trypsin digestion ${ }^{39}$, from both wildtype and STUB1inactivated cells. Then, by mass spectrometry, we identified differentially ubiquitinated lysine residues between the two genotypes. From this analysis, we learned that IFNY$\mathrm{R} 1^{\mathrm{K} 285}$ and $\mathrm{JAK} 1^{\mathrm{K} 249}$ were more frequently ubiquitinated in STUB1-deficient cells (Fig. 3e).

This raises the possibility that STUB1 specifically recognizes these ubiquitinated residues and uses them as substrates for subsequent proteasomal degradation of their respective proteins. To validate this hypothesis, we generated melanoma cell clones deficient in both IFNGR1 and JAK1 (IFNGR1-KO + JAK1-KO) in either a wildtype or STUB1-deficient background. We then reconstituted JAK1 and IFNGR1 either in a wildtype configuration, or in a form in which the STUB1-targeted lysine residues were mutated to arginine, thereby precluding ubiquitination events from occurring at those sites. We assessed the effects of the various mutations and 
genotypes on IFNY-R1 and JAK1 protein levels by flow cytometry and Western blot

(Fig. 3f-k and Supplementary Fig. 3f-i). This reconstitution experiment showed that preventing ubiquitination of IFNY-R $1^{\mathrm{K} 285}$ and $\mathrm{JAK} 1^{\mathrm{K} 249}$ resulted in marked protein stabilization of IFNY-R1 and JAK1 in wildtype cells (Fig. $\mathbf{3 g}$ and Supplementary Fig. 3f-i, sgCtrl samples). This increased protein stability of mutant IFNY-R $1^{\mathrm{K} 285}$ and JAK $1^{\mathrm{K} 249}$ likely occurs through reduced proteasomal turnover, as MG132 treatment was unable to further stabilize IFNy-R1 and JAK1 levels in the IFNy-R $1^{\mathrm{K} 285}$ and JAK $1^{\mathrm{K} 249}$ mutants, whereas it did in wildtype cells (Fig. 3g-i).

To assess the reliance of STUB1 on these residues for modifying IFNY-R1 and JAK1 stability, we continued by inactivating STUB1 in the IFNY-R $1^{\mathrm{K} 285}$ and JAK1 ${ }^{\mathrm{K} 249}$ mutant cells. We analyzed IFNY-R1 and JAK1 expression by Western blot (Fig. 3j and Supplementary Fig. 3f-h) and additionally assessed IFNY-R1 expression by flow cytometry (Fig. 3k and Supplementary Fig. 3i). Whereas in STUB1-proficient cells, the IFNY-R1 ${ }^{\mathrm{K} 285}$ and $\mathrm{JAK} 1^{\mathrm{K} 249}$ mutants resulted in increased stability of IFNY-R1 and JAK1 (Fig. 3j, k and Supplementary Fig. 3f-i), they were unable to further increase IFNY-R1 and JAK1 in a STUB1-KO background (Fig. 3j, k and Supplementary Fig. $3 \mathbf{g}$ and $\mathbf{h}$ ). This finding suggests that STUB1 recognizes and requires the ubiquitinated residues IFNy-R $1^{\mathrm{K} 285}$ and $\mathrm{JAK} 1^{\mathrm{K} 249}$ to target their parent proteins, IFNy-R1 and JAK1, for proteasomal degradation (Fig. 3I).

STUB1 inactivation sensitizes melanoma cells to cytotoxic $\mathrm{T}$ cells through amplified IFNy signaling

Having established that STUB1 regulates IFNY-R1 and JAK1 expression under homeostatic conditions, we next asked whether this regulation affects receptor complex stability during active IFNy signaling. Whereas wildtype tumor cells moderately upregulated IFNy-R1 expression upon treatment with increasing amounts of IFNy, STUB1-deficient cells further elevated IFNy-R1 protein levels, particularly the heavier, cell-surface isoforms (Fig. 4a). We also observed this altered IFNy response in STUB1-deficient cells with downstream mediators of IFNy signaling, as illustrated by an accelerated and robust onset of STAT1 phosphorylation upon IFNy treatment in STUB1-depleted cells (Fig. 4b). This altered signaling translated into enhanced transcription of IFNy-responsive genes, such as CD274 (encoding PD-L1; Fig. 4c) 
and IDO1 (Supplementary Fig. 4a). We confirmed this observation at the protein level (Supplementary Fig. $4 \mathrm{~b}$ and c).

In light of these results, it was important to assess whether this hyperresponsiveness to IFNy would also alter how STUB1-deficient tumor cells respond to T cell attack. We therefore profiled transcriptomic changes in wildtype and STUB1-deficient melanoma cells after T cell attack (Fig. 4d). Gene set enrichment analysis (GSEA) revealed that STUB1-depleted melanoma cells exhibit an amplified IFNy response compared to wildtype cells (Fig. 4e), whereas, as a control for its specificity, genes within the TNF pathway did not show this enrichment (Fig. 4e). We additionally derived an experimental IFNy response gene set from IFNy-treated melanoma cells (Supplementary Fig. 4d). This gene set was significantly stronger induced in STUB1deficient melanoma cells challenged with cytotoxic $T$ cells than in its control counterpart (Fig. 4f, g). We confirmed this effect in a second cell line (Supplementary Fig. 4e and f). Additionally, this effect was specific to IFNy-signaling, as it did not occur for a TNF signaling-based gene set (Fig. 4f, g; Supplementary Fig. 4e and f).

Given these findings, and our previous results demonstrating that elevated IFNY-R1 levels sensitize tumor cells to IFNy treatment and cytotoxic T cells, we next tested whether STUB1 inactivation induces hypersensitivity to (T cell-derived-) IFNy. Indeed, at concentrations where wildtype melanoma cells were barely affected by IFNy or T cell attack, STUB1-deficient melanoma cells were eliminated efficiently (Fig. $\mathbf{4 h - k}$ and Supplementary Fig. $\mathbf{4 g - j}$ ). We confirmed that the sensitization to T cell attack was IFNY-dependent, as both STUB1-deficient and wildtype cells were equally sensitive to T cell attack when lacking IFNY-R1 expression (Fig. 4I, m, and Supplementary Fig. $\mathbf{4 k}$ and I). Collectively, these data show that the strong basal and dynamic induction of IFNY-R1 expression by STUB1 inactivation results in intensified IFNY signaling and consequently, IFNy-dependent sensitization of melanoma cells to cytotoxic $\mathrm{T}$ cells in vitro.

\section{STUB1 inactivation and anti-PD-1 treatment constitute a rational combination} therapy approach

Having observed an enhanced sensitivity of STUB1-deficient melanoma cells to cytotoxic T cell pressure in vitro (Fig. 4), we next investigated whether this is 
recapitulated cross-species and in vivo. We first established Stub1-deficient murine melanoma cell lines in which we could reiterate our findings from human cell lines in vitro (Supplementary Fig. 5a-e). Importantly and in line with our in vitro data, we validated that immunogenic B16F10-dOVA tumors lacking Stub1 induced PD-L1 to a greater extent than wildtype tumors in vivo (Fig. 5a-c).

This provided a rationale to combine Stub1 inactivation with anti-PD-1 treatment, as that combination would allow for intensified IFNy signaling while simultaneously preventing PD-L1-mediated immune evasion. To experimentally test this, we differentially labeled wildtype and Stub1-deficient B16F10-dOVA cells with either EGFP or mCherry, respectively. We then mixed these cell lines in a 1:1 ratio and transplanted them into immune-deficient NSG mice, or into immune-proficient C57BL/6 mice that were subsequently treated with either an isotype control antibody or an anti-PD-1 antibody. After 12 days, tumors were harvested and the ratio between

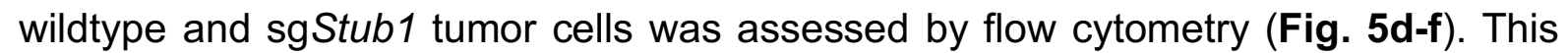
analysis indicated that while there was a trend towards higher sensitivity of Stub1deficient tumors to immune attack (Fig. 5e, f, compare NSG vs. aISO), strong depletion of Stub1-deficient tumors was observed only upon treatment with anti-PD-1 antibodies (Fig. 5e, f, compare NSG vs. aPD-1 and aISO vs. aPD-1). This finding illustrates the rationale for combining STUB1 inhibition with anti-PD-1 therapy: it would allow for the increased susceptibility of tumors to T cell-derived IFNy, yet at the same time block the negative effects of increased IFNy signaling, namely increased PD-L1 levels.

To substantiate the notion that STUB1 inhibition and anti-PD-1 treatment constitute a rational treatment combination, we integrated our experimental data with clinical transcriptomic data. Based on the transcriptomic data we obtained from wildtype and STUB1-deficient melanoma cells after T cell attack (Fig. 4d), we established a STUB1KO signature based on differentially upregulated genes in STUB1-deficient melanoma cells compared to wildtype cells upon T cell challenge (Table 1). We then applied this signature to transcriptomic data of melanoma patients undergoing different ICB therapies $^{12,40}$. We found that a high STUB1-KO signature expression was associated with response to anti-PD-1 treatment in two cohorts (Fig. $\mathbf{5 g}, \mathbf{h}$ and Supplementary Fig. 5f, g). Such correlation was not found for anti-CTLA-4 treatment (Fig. 5i and j). 
359 Importantly, these associations were not biased by the limited presence of classical

360 IFNy response genes in the STUB1-KO signature (Supplementary Fig. 5h-j).

361 Collectively, these findings support the notion that STUB1 inactivation in combination

362 with anti-PD-1 treatment represents a rational combinatorial treatment approach. 


\section{Discussion}

365 Although the importance of IFNy signaling in immunotherapy has become apparent in

366 recent years, both experimental and preclinical studies have been largely focusing on

367 perturbations in this pathway that contribute to tumor immunogenicity editing and

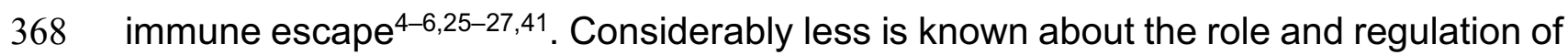

369 IFNy-R1 cell surface expression levels, particularly whether and how increased

370 abundance sensitizes to (T cell-derived) IFNy. We show here that heightened IFNy-

371 R1 expression levels on tumor cells increases the susceptibility to T cell-derived IFNy

372 and its antitumor activity. This observation is underscored by clinical data strongly

373 linking transcriptional IFNy-dependent signaling in tumors to ICB therapy

374 response ${ }^{11,17,24}$.

The relationship between IFNy-R1 levels, IFNy signaling and immune sensitivity raises the possibility that induction of this pathway may trigger immune responsiveness of tumor cells, something that may be therapeutically explored. Because little is known about mechanisms governing IFNY-R1 cell surface expression, we performed an unbiased genome-wide screen and uncovered STUB1 as the most prominent hit: its loss led to increased IFNy-receptor complex cell surface expression. STUB1 acts by mediating proteasomal degradation of its core components, IFNY-R1 and its interaction partner JAK1. Our results suggest that STUB1 is a conserved E3 ubiquitin ligase for both IFNy-R1 and JAK1, extending a previous observation on the ubiquitination of IFNy-R1 ${ }^{42}$. While STUB1 loss stabilizes cell surface IFNy-R1, it also increases the abundance of JAK1. We show that, in turn, the increased abundance of JAK1 has a stabilizing effect on IFNy-R1, because ectopic expression of JAK1 was sufficient to strongly stabilize IFNy-R1. This finding was rather unexpected, given that JAK1 is believed to function solely as a kinase downstream of IFNY-R1 following ligand engagement. Our results indicate that JAK1 in IFNy receptor signal transduction is more influential.

Mechanistically, the identification of the critical ubiquitinated lysine residues, which STUB1 uses for ubiquitination of its IFNy receptor targets, IFNy-R1 ${ }^{\mathrm{K} 285}$ and JAK1 ${ }^{\mathrm{K} 249}$, is of relevance to understand this mode of regulation. IFNY-R $1^{\mathrm{K} 285}$ is located in the box

3961 motif that is shared among cytokine class II receptors and is critical for JAK1 397 binding ${ }^{43}$. Conversely, $\mathrm{JAK} 1^{\mathrm{K} 249}$ is located in the complementary FERM-domain of 
JAK1, enabling the binding to the box1 motif of IFNY-R143. These observations raise the possibility that JAK1 stabilizes IFNY-R1 by masking the critical IFNY-R1 ${ }^{\mathrm{K} 285}$ residue prone to ubiquitination and thereby prevents subsequent STUB1-mediated proteasomal degradation. As demonstrated above, this regulatory mechanism may become even more apparent when IFNy engages with its cognate receptor. Interestingly, this ubiquitination-mediated control of IFNy signaling at the level of IFNYR1 may constitute a more common mechanism, as recently another ubiquitin ligase, FBXW7, was implicated in governing IFNY-R1 signaling in breast cancer ${ }^{44}$. Our findings are complementary to this study; together they not only uncover the importance of ubiquitin-mediated IFNy-R1 modulation but also highlight the unexpectedly broad consequences of this type of regulation, with strong effects in tumor cells ranging from heightened immune sensitivity to metastasis.

Our data suggest that as a result of IFNY-R1 stabilization, STUB1 loss leads to enhanced IFNy response as well as strong sensitization to cytotoxic T cell-mediated tumor cell killing. This suggests that the physiological role for STUB1 is to dampen IFNy responses. Our findings therefore explain several previous observations. First, STUB1 inactivation was found to sensitize tumors to immune pressure in the context of GVAX and anti-PD-1 therapy ${ }^{26}$; however, the underlying mechanism of this observation was unknown. Second, in a previous genome-wide loss-of-function screen for IFNy signaling-independent tumor immune sensitizers, STUB1 was not identified as a hit $^{19}$, highlighting its specific role as modulator of IFNy signaling. Third, STUB1 was identified as a regulator of IFNy-induced PD-L1 expression ${ }^{45}$. It was postulated that STUB1 directly mediates proteasomal degradation of PD-L1. However, we demonstrate that, instead, STUB1 acts as a modulator of IFNy signaling and thus indirectly modulates PD-L1 expression.

In clinical trials, PD-1 blockade is now being combined with a genuine plethora of secondary treatments, although the rationale is not always fully clear from the available experimental evidence ${ }^{46}$. We show that STUB1 loss leads to an enhanced IFNy-dependent transcriptional program. From a therapeutic point of view this could be beneficial, because several IFNy target genes, such as HLA, contribute to tumor eradication. However, also PD-L1 represents an established IFNy target, which we confirm here, and this constitutes an immune-protective tumor trait. Our observations, 
432 therefore, provide a clear rationale for combining STUB1 perturbation with PD-1

433 blockade. Indeed, we show that STUB1 deficiency in tumors synergizes with anti-PD-

4341 treatment in a murine model of melanoma. Collectively, our results therefore merit

435 clinical exploration of inhibiting STUB1 in combination with PD-1 blockade, which will 436 require the development of a pharmacologic inhibitor.

437 
Materials and Methods

Cell lines used in the study

440 The human D10 (female), SK-MEL-23 (female), SK-MEL147 (female), A375 (female), SK-MEL-28 (male), BLM-M (male), 451Lu (male), A101D (male), LCLC-103H (male), HCC-4006 (male), RKO (unspecified), 8505C (female) and HEK293T (female) cell lines were obtained from the internal Peeper laboratory stock, as was the murine B16F10-OVA (male) cell line. The murine D4M.3A (male) cell line was obtained from the Blank laboratory. All cell lines were tested monthly by PCR to be negative for mycoplasma infection.

MART-1 T cell generation

MART-1 retrovirus was made using a producer cell line as described previously ${ }^{47}$. Peripheral blood mononuclear cells (PBMCs) were isolated from healthy donor buffy coats (Sanquin, Amsterdam, the Netherlands) by density gradient centrifugation using Lymphoprep (Stem cell technologies, \#07801). CD8 T cells were purified from the PBMC fraction using CD8 Dynabeads (Thermo Fisher Scientific, 11333D) according to manufacturer's instructions. The isolated CD8 T cells were activated for 48 hours on non-tissue culture-treated 24-well-plates, which had been coated with anti-CD3 and anti-CD28 activating antibodies overnight (eBioscience, 16-0037-85, 16-0289-85, each $5 \mu \mathrm{g}$ per well) at a density of $2 \times 10^{6}$ cells per well. After 48 hours $2 \times 10^{6}$ cells were harvested and mixed with the MART-1 virus at a 1:1 ratio and plated on a non-tissue culture-treated 24-well-plate, which had been coated with Retronectin overnight (Takara Bio, TB T100B, $25 \mu \mathrm{g}$ per well). Spinfection was performed for two hours at 2000g. 24 hours following spinfection, MART-1 CD8 T cells were harvested and cultured for seven days, after which the transduction efficiency was assessed by flow cytometry using anti-mouse TCR $\beta$ (BD Bioscience, 553174). CD8 T cells were cultured in RPMI (Gibco, 11879020) containing 10\% human serum (One Lamda, A25761), 100 units $/ \mathrm{ml}$ penicillin, $100 \mu \mathrm{g}$ per $\mathrm{ml}$ Streptomycin, 100 units $/ \mathrm{ml}$ IL-2 (Proleukin, Novartis), $10 \mathrm{ng} / \mathrm{ml}$ IL-7 (ImmunoTools, 11340077) and $10 \mathrm{ng} / \mathrm{ml}$ IL-15 
In vitro tumor competition assay

IFNY-R1 $1^{\text {Low }}$ and IFNY-R1 $1^{\text {High }}$-expressing tumor cells were labelled with CellTrace CFSE Cell Proliferation Dye (CFSE, Thermo Fisher Scientifc, C34554) or CellTrace Violet Cell Proliferation Dye (CTV, Thermo Fisher Scientific, C34557) according to manufacturer's instructions. The labeled tumor cells were mixed in a 1:1 ratio and $4 \times 10^{6}$ cells were seeded per $10 \mathrm{~cm}$ dish (Greiner). The tumor cell mix was subsequently challenged three times for 24 hours with either MART-1 T cells or control T cells at a 1:8 ratio. In parallel, the tumor cell mix was treated with either $25 \mathrm{ng} / \mathrm{ml}$ IFNy or vehicle for five days. The surviving tumor cell fraction was analyzed for CFSE and CTV staining by flow cytometry 24 hours after the final T cell challenge or after five days of IFNy treatment.

\section{IFNY-induced PD-L1 and MHC class I expression}

Tumor cells were seeded in 24-well-plates at a density of $3 \times 10^{5}$ cells per well and treated either with a serial dilution series of IFNy (PeproTech, 300-02) (starting at 50 $\mathrm{ng} / \mathrm{ml}$ in two-fold dilution steps) or vehicle for 24 hours. The cells were harvested after treatment and stained for PD-L1 (eBioscience, 12-5983-42) and MHC class I (R\&D Systems, FAB7098G). Induction of the respective proteins was analyzed by flow cytometry.

\section{Lentiviral transductions}

HEK293T cells were co-transfected with pLX304 plasmids containing constructs of interest and the packaging plasmids pMD2.G (Addgene, \#12259) and psPAX (Addgene, \#12260) using polyethylenimine. 24 hours after transfection, the medium was replaced with OptiMEM (Thermo Fisher, 31985054) containing 2\% fetal bovine serum. Another 24 hours later, lentivirus-containing supernatant was collected, filtered and stored at $-80^{\circ} \mathrm{C}$. Tumor cells were lentivirally transduced by seeding $5 \times 10^{5}$ cells per well in a 12-well plate (Greiner), adding lentivirus at a 1:1 ratio. After 24 hours the virus-containing medium was removed and transduced tumor cells were selected with antibiotics for at least seven days.

\section{Sort-based genome-wide CRISRP/Cas9 knockout screen}

D10 and SK-MEL-23 melanoma cells were first transduced to stably express Cas9 (lentiCas9-Blast, Addgene, \#52962) and selected with blasticidin (5 $\mathrm{gg} / \mathrm{ml}$ ) for at least 
ten days. The respective cell lines were subsequently transduced with the human genome-wide CRISPR-KO (GeCKO, Addgene, \#1000000048, \#1000000049) sgRNA library at a 1000 -fold representation and a multiplicity of infection of $<0.3$ to ensure one sgRNA integration per cell. The library transduction was performed in two replicates per cell line. Transduced cells were selected with puromycin $(1 \mu \mathrm{g} / \mathrm{ml})$ for two days, after which library reference samples were harvested. Cells were cultured for an additional eight days to allow gene inactivation and establishment of the respective phenotype. Before sorting, a pre-sort bulk population was harvested.

514 Library-transduced cells were then harvested and stained with anti-IFNy-R1/CD119APC antibody (Miltenyi Biotech, 130-099-921) for FACSorting. From the live cell population $10 \%$ of cells with the highest and $10 \%$ of cells with the lowest IFNy-R1 expression were sorted. The sorted cells were washed with PBS and the cell pellet was snap frozen. Genomic DNA was isolated using the Blood and Cell culture MAXI Kit (Qiagen, 13362), according to manufacturer's instructions. sgRNAs were amplified using a one-step barcoding PCR using NEBNext High Fidelity 2X PCR Master Mix (NEB, M0541L) and the following primers: Forward primer: 5 ATCTNNNNNNGGCTTTATATATCTTGTGGAAAGGACGAAACACC-3' Reverse Primer: 5'-CAAGCAGAAGACGGCATACGAGATCCGACTCGGTGCCACTTTTTCAA-3'

The hexa- $\mathrm{N}$ nucleotide stretch contains a unique barcode to identify each sample following deep sequencing. MAGeCK (v0.5.6) was used to perform the analysis of the screen. To assess the depletion of core essential genes we compared the library reference sample to the pre-sorted bulk population. Putative regulators of IFNy-R1 were identified by comparing the sgRNA abundance among the $10 \%$ highest and lowest IFNy-R1-expressing populations and a signed robust rank aggregation (RRA) score was assigned to the respective genes. sgRNA targets with a false discovery rate $($ FDR $)<0.25$ were considered as putative hits. 
qPCR-based detection of transcriptomic differences

541 RNA from D10, SK-MEL-147 and SK-MEL-23 melanoma cells expressing either sgCtrl 542 or sgSTUB1 was isolated using the Isolate II RNA Mini Kit (Bioline, BIO-52072) 543 according to manufacturer's instructions. cDNA was reverse transcribed using the

544 Maxima First Strand cDNA synthesis kit (Fisher Scientific, 15273796) according to 545 manufacturer's instructions. cDNA samples were probed for the expression of RPL13, 546 IFNGR1, JAK1, CD274 and IDO1 using the following primers:

547 RPL13:

548 Forward: 5'- GAGACAGTTCTGCTGAAGAACTGAA-3'

549 Reverse: 5'- TCCGGACGGGCATGAC-3'

550 IFNGR1:

551 Forward: 5'-CGGAAGTGACGTAAGGCCG-3'

552 Reverse: 5'-TTAGTTGGTGTAGGCACTGAGGA-3'

553 JAK1:

554 Forward: 5'- TACCACGAGGCCGGGAC-3'

555 Reverse: 5'- AGAAGCGTGTGTCTCAGAAGC-3'

556 CD274:

557 Forward: 5'- TGGCATTTGCTGAACGCATTT-3'

558 Reverse: 5'- AGTGCAGCCAGGTCTAATTGTT-3'

559 IDO1:

560 Forward: 5'- AATCCACGATCATGTGAACCCA-3'

561 Reverse: 5'- GATAGCTGGGGGTTGCCTTT-3'

563 Gene Expression was quantified using the SensiFAST SYBR Hi-Rox Kit (Bioline, 564 92090) in combination with the StepOnePlus Real-Time PCR System (Thermo 565 Fisher). Gene expression was normalized to RPL13 expression using the $\triangle \triangle \mathrm{Ct}$ 566 approach.

T cell-melanoma cell co-culture

569 Depending on the melanoma cell line, $5 \times 10^{4}$ to $1.2 \times 10^{5}$ cells were seeded per well in 570 12-well plates in $0.5 \mathrm{ml}$ DMEM containing $10 \%$ FBS. Melanoma cells were 571 subsequently either co-cultured with the equivalent amount of control T cells or a serial 572 dilution of MART-1 T cells in $0.5 \mathrm{ml}$ DMEM containing 10\% FBS (starting with a 1:1 573 ratio and two-fold dilution steps). After 24 hours $T$ cells were removed by washing the 
plates with PBS, fresh culture medium was added and the melanoma cells were grown was removed and all wells were fixed with methanol and stained with crystal violet $(0.1 \%)$ for 30 minutes.

578 B16F10-OVA cells were seeded at a density of $5 \times 10^{4}$ cells per well in $0.5 \mathrm{ml}$ DMEM containing $10 \%$ FBS in 12-well plates. OT-I T cells were then added in a two-fold serial dilution starting from 4:1 ( $T$ cell : melanoma cell) ratio in $0.5 \mathrm{ml}$ DMEM containing 10\% FBS. After 48 hours OT-I T cells were removed by washing the wells with PBS. The remaining melanoma cells were grown for an additional 48 hours, before being fixed with methanol and stained with crystal violet $(0.1 \%)$. The crystal violet was removed and the plates were washed with water. After image acquisition, the crystal violet was suspended using a $10 \%$ acetic acid solution and the optical density of the resulting suspension was quantified.

\section{Protein expression analysis by immunoblot}

589 Whole cell lysates were generated by removing culture medium and washing the adherent cells on the plate twice with PBS. The cells were then scraped, harvested in $1 \mathrm{ml}$ PBS and pelleted by centrifugation at $1000 \mathrm{~g}$. After removing PBS, the cell pellet was resuspended into the appropriate amount of RIPA lysis buffer (50mM TRIS pH 8.0, $150 \mathrm{mM} \mathrm{NaCl}, 1 \%$ Nonidet $\mathrm{P} 40,0.5 \%$ sodium deoxycholate, $0.1 \%$ SDS) supplemented with HALT Protease and Phosphatase inhibitor cocktail (Fisher Scientific, 78444). Lysis was performed on ice for 30 minutes. The samples were subsequently centrifuged at $17,000 \mathrm{~g}$ and whole cell lysates were collected. The protein content of each lysate was quantified using Bio-Rad protein assay (Bio-Rad, 500-0006). Protein concentrations were equalized and immunoblot samples were prepared through addition of 4xLDS sample buffer (Fisher Scientific, 15484379) containing $10 \% \quad \beta$-Mercaptoethanol (final concentration 2.5\%) and subsequent incubation of the samples at $95^{\circ} \mathrm{C}$ for five minutes. Proteins in lysates were sizeseparated using 4-12\% Bis-Tris polyacrylamide-SDS gels (Life Technologies) and nitrocellulose membranes (GE Healthcare). Blots were blocked using 4\% Milk powder in $0.2 \%$ Tween-20 in PBS. Blocked membranes were incubated with primary 605 antibodies overnight. Immunoblots were developed using Super Signal West Dura 606 Extended Duration Substrate (Thermo Fisher, 34075). Luminescence signal was 
captured by Amersham Hyperfilm high performance autoradiography film or by the Bio-Rad ChemiDoc imaging system. The following primary antibodies were used antiIFNy-R1 (Santa Cruz Biotechnology, sc-28363), anti-JAK1 (D1T6W, Cell Signaling

610 Technology, 50996), anti-STUB1/CHIP (C3B6, Cell Signaling Technology, 2080), anti-

611 Tubulin (DM1A, Sigma Aldrich, T9026), anti-STAT1 (D1K9Y, Cell Signaling 612 Technology, 12994), anti-STAT1-Tyr701 (58D6, Cell Signaling Technology, 9167), 613 anti-mouse PD-L1 (MIH5, Thermo Fisher Scientific, 14-5982-81).

614

\section{Quantification of protein expression of immunoblots}

616 Protein expression on immunoblots was quantified on 8-bit gray-scale-transformed .tiff 617 images of either scanned Amersham Hyperfilm MP (GE Healthcare, 28906838) or .tiff 618 images obtained by the Bio-Rad ChemiDoc imaging system. Fiji ImageJ was used to 619 select a region of interest for the respective proteins. Protein expression for each protein was normalized to the loading control of the respective sample.

Biotin labeling of cell surface proteins

623 Biotin labeling of cell surface proteins was performed according to the published protocol published by Huang ${ }^{31}$. In brief, $2 \times 10^{6}$ D10 melanoma cells were seeded in 10 $\mathrm{cm}$ culture dish 48 hours prior to the experiment. Cells were washed twice in ice-cold $\mathrm{PBS} / \mathrm{CaCl}_{2} / \mathrm{MgCl}_{2}(+2.5 \mathrm{mM} \mathrm{CaCl}, 1 \mathrm{mM} \mathrm{MgCl}$, $\mathrm{pH}$ 7.4). Cell surface proteins were labeled with $2 \mathrm{ml}$ of $0.5 \mathrm{mg} / \mathrm{ml}$ Sulfo-NHS-SS-biotin (in $\mathrm{PBS} / \mathrm{CaCl}_{2} / \mathrm{MgCl}_{2}$ ) on ice for 30 minutes. Labeling was quenched by washing cells three times with $3 \mathrm{ml}$ of $50 \mathrm{mM}$ glycine (in PBS/CaCl$/ \mathrm{MgCl}_{2}$ ). Cells were lysed using RIPA lysis buffer and biotinylated proteins were pulled down using Streptavidin-coated magnetic beads. Samples were size-separated using 4-12\% Bis-Tris polyacrylamide-SDS gels (Life Technologies) and nitrocellulose membranes (GE Healthcare). And immunoblotted for

633 IFNy-R1.

\section{Proteome profiling}

636 sgCtrl- and sgSTUB1-expressing D10 and SK-MEL-147 melanoma cells (triplicates 637 for both conditions) were lysed in 8M urea lysis buffer in the presence of cOmplete Mini protease inhibitor (Roche) and aliquots of $200 \mu \mathrm{g}$ protein were reduced, alkylated with chloroacetamide, predigested with Lys-C (Wako) $\left(1: 75,4 \mathrm{~h}\right.$ at $\left.37^{\circ} \mathrm{C}\right)$ and trypsin 640 digested overnight (Trypsin Gold, Mass Spectrometry Grade, Promega; 1:50 at $37^{\circ} \mathrm{C}$ ). 
641 Peptide samples were desalted using C18 Sep-Pak cartridges (3cc, Waters) and eluted with acidic $40 \%$ and $80 \%$ acetonitrile. Dried D10 and SK-MEL-147 digests were reconstituted in 50mM HEPES buffer and replicates were labeled with 10-Plex TMT reagent (Thermo Fisher Scientific) according to the manufacturer's instructions. Labeled samples were mixed equally for both cell lines, desalted using Sep-Pak C18 cartridges and fractionated by basic reversed-phase (HpH-RP) HPLC separation on a Phenomenex Gemini C18 analytical column (100 mm x $1 \mathrm{~mm}$, particle size $3 \mu \mathrm{m}, 110$ Å pores) coupled to an Agilent 1260 HPLC system over a 60 minute gradient. Per cell line, fractions were concatenated to 12 fractions for proteome analysis. hybrid mass spectrometer (Q-OT-qIT, Thermo Scientific) equipped with an EASY-NLC 1000 system (Thermo Scientific). Samples were directly loaded onto the analytical column (ReproSil-Pur $120 \mathrm{C} 18-\mathrm{AQ}, 1.9 \mu \mathrm{m}, 75 \mu \mathrm{m} \times 500 \mathrm{~mm}$, packed in-house). Solvent $A$ was $0.1 \%$ formic acid/water and solvent $B$ was $0.1 \%$ formic $a c i d / 80 \%$ acetonitrile. Samples were eluted from the analytical column at a constant flow of 250 $\mathrm{nl} / \mathrm{min}$ in a four-hour gradient containing a 120 -minute increase to $24 \%$ solvent $\mathrm{B}$, a 60 -minute increase to $35 \% \mathrm{~B}$, a 40 -minute increase to $45 \% \mathrm{~B}, 20$-minute increase to $60 \% \mathrm{~B}$ and finishing with a 15-minute wash. MS settings were as follows: full MS scans $(375-2000 \mathrm{~m} / \mathrm{z})$ were acquired at 120,000 resolution with an AGC target of $4 \times 10^{5}$ charges and maximum injection time of $50 \mathrm{~ms}$. The mass spectrometer was run in top speed mode with 3s cycles and only precursors with charge state 2-7 were sampled for MS2 using 60,000 resolution, MS2 isolation window of $1 \mathrm{Th}, 5 \times 10^{4}$ AGC target, a maximum injection time of $60 \mathrm{~ms}$, a fixed first mass of $110 \mathrm{~m} / \mathrm{z}$ and a normalized collision energy of $33 \%$. Raw data files were processed with Proteome Discoverer 2.2 (Thermo Fisher Scientific) using a Sequest HT search against the Swissprot reviewed human database. Results were filtered using a 1\% FDR cut-off at the protein and peptide level. TMT fragment ions were quantified using summed abundances with PSM filters requiring a $S / N \geq 10$ and an isolation interference cutoff of $35 \%$. Normalized protein and peptide abundances were extracted from PD2.2 and further analyzed using Perseus software (ver. 1.5.6.0) ${ }^{48}$. Differentially expressed proteins were determined using a t-test (cutoffs: $p<0.05$ and LFQ abundance difference $<-0.2^{\wedge}>$ 
675

676

677

678

679

680

681

682

683

684

685

686

687

688

689

690

691

692

693

694

695

696

697

698

699

700

701

702

703

704

705

706

707

708

\section{Ubiquitination site profiling}

For ubiquitination site profiling, D10 melanoma cells expressing either a non-targeting control sgRNA (sgCtrl) or sgSTUB1 were lysed in 8M urea lysis buffer in the presence of cOmplete Mini protease inhibitor (Roche). Triplicates corresponding to $14 \mathrm{mg}$ protein per sample for sgCtrl and sgSTUB1-expressing D10 cells were reduced, alkylated with chloroacetamide, predigested with Lys-C (Wako) $\left(1: 75\right.$, 4h at $37^{\circ} \mathrm{C}$ ) and trypsin digested overnight (Trypsin Gold, Mass Spectrometry Grade, Promega; 1:50 at $37^{\circ} \mathrm{C}$ ). Peptide samples were desalted using C18 Sep-Pak cartridges (3cc, Waters) and eluted with acidic $40 \%$ and $80 \%$ acetonitrile. At this stage, aliquots corresponding to $200 \mu \mathrm{g}$ protein digest were collected for proteome profiling, the remainder of the eluates being reserved for enrichment of ubiquitinated peptides. All peptide fractions were vacuum dried and stored at $-80^{\circ} \mathrm{C}$ until further processing. Ubiquitinated peptides were enriched by immunoaffinity purification using the PTMScan Ubiquitin Remnant Motif (K- $\varepsilon-G G)$ Kit (Cell Signaling Technology, 5562) according to the manufacturer's instructions. Ubiquitinated peptide samples were analyzed by nanoLC-MS/MS on an Orbitrap Fusion Tribrid mass spectrometer equipped with a Proxeon nLC1000 system (Thermo Scientific) using a non-linear 210 minute gradient as described previously ${ }^{49}$. Raw data files were processed with MaxQuant (ver. 1.5.6.0) ) $^{50}$, searching against the human reviewed Uniprot database (release 2018_01). False discovery rate was set to $1 \%$ for both protein and peptide level and $G G(K)$ was set as additional variable modification for analysis of ubiproteome samples. Ubiquitinated peptides were quantified with label-free quantitation (LFQ) using default settings. LFQ intensities were Log $_{2}$-transformed in Perseus (ver. 1.5.6.0) ${ }^{48}$, after which ubiquitination sites were filtered for at least two valid values (out of 3 total) in at least one condition. Missing values were replaced by an imputation-based normal distribution using a width of 0.3 and a downshift of 1.8. Differentially regulated ubiquitination sites were determined using a t-test (thresholds: $p<0.05$ and LFQ abundance difference $<-1.0^{\wedge}>1.0$ ).

\section{Proteasomal inhibitor treatment}

Melanoma cells were seeded and grown to $80 \%$ confluence and treated with either DMSO (vehicle) or with $10 \mu M$ MG132 (Medchem Express, HY-13259) for four hours. The medium was removed four hours later, cells were washed three times with PBS and whole cell lysates were prepared as described above. 
Animal studies

All animal studies were approved by the animal ethics committee of the Netherlands Cancer Institute (NKI) and performed in accordance with ethical and procedural guidelines established by the NKI and Dutch legislation. Male mice, of either C57BL/6 (Janvier) or NSG-B2m (The Jackson Laboratory) mouse strains were used at an age of 8-12 weeks.

In vivo tumor competition assay

717 B16F10-dOVA cells were lentivirally transduced with lenti-Cas9-blast to stably express 718 Cas 9 and selected with blasticidin $(5 \mu \mathrm{g} / \mathrm{ml})$ for at least ten days. The cells were then 719 lentivirally transduced to stably express either sgCtrl or sgStub1 (lentiGuide-Puro, \#52963) and cultured with puromycin $(1 \mu \mathrm{g} / \mathrm{ml})$ for at least ten days to allow for selection of cells with genetic inactivation of Stub1. Knockout efficiency was assessed by immunoblotting. sgCtrl-expressing cells were transduced to stably express EGFP (pLX304-EGFP-Blast) and sgStub1-expressing cells were transduced to stably express mCherry (pLX304-mCherry-Blast). EGFP and mCherry-positive populations were sorted and cultured. Cells were mixed in a 1:1 ratio prior to injection and $5 \times 10^{5}$ cell per mouse were injected into immune-deficient NSG- $\beta 2 m^{-/-}(n=10$, The Jackson Laboratory, 010636; RRID:ISMR_JAX:010636), or C57BL/6J mice ( $n=20$, Janvier, C57BL/6JRj). Tumor bearing C57BL/6J mice were treated with either $100 \mu \mathrm{g} / \mathrm{mouse}$ isotype control antibody (Leinco Technologies, R1367) or with $100 \mu \mathrm{g} / \mathrm{mouse}$ antimouse-Pd-1 (Leinco Technologies, P372) one and six days post tumor injection. Tumors were harvested at day 12 and dissociated into single cell suspensions. Cells were subsequently stained for immune cells using anti-CD45-APC (Miltenyi, 130-102$544)$ and the tumor composition was analyzed by flow cytometry.

\section{Transcriptomic profiling of melanoma cells after T cell attack} 2x10 D10 and SK-MEL-147 melanoma cells were plated per dish in $10 \mathrm{~cm}$ cell culture dishes 48 hours prior to $\mathrm{T}$ cell challenge. Melanoma cells were subsequently challenged with either Ctrl or MART-1 T cells for eight hours. The T cells were removed by washing the plates with PBS. The remaining tumor cells were harvested and lysed in RLT buffer (Qiagen, 79216) and sequenced on an Illumina HiSeq2500. Fastq files were mapped to the human reference genome (Homo.sapiens.GRCh38.v77) using

742 Tophat v2.151 with default settings for single-end data. The samples were used to 
743

744

745

746

747

748

749

750

751

752

753

754

755

756

757

758

759

760

761

762

763

764

765

766

767

768

769

770

771

772

773

774

775

generate read count data using itreecount (github.com/NKI-GCF/itreecount).

Normalization and statistical analysis of the expression of genes was performed using DESeq2 (V1.24.0 $)^{52}$. Centering of the normalized gene expression data was performed by subtracting the row means and scaling by dividing the columns by the standard deviation (SD) to generate a Z-score.

Differentially expressed genes between STUB1-deficient and wildtype cells were calculated with DESeq $2^{52}$ using FDR $<0.01$. The significant genes that were upregulated comprise the STUB1-KO signature (Table 1).

\section{External Datasets}

The anti-PD-1 treated melanoma patient samples were taken from Riaz et al. ${ }^{12}$ (ENA/SRA database: PRJNA356761). Fastq files were downloaded and mapped to the human reference genome (Homo.sapiens.GRCh38.v82) using STAR(2.6.0c) ${ }^{53}$ in 2-pass mode with default settings for paired-end data. The samples were used to generate read count data using HTSeq-count ${ }^{54}$. Normalization and statistical analysis of the expression of genes was performed using DESeq2 ${ }^{52}$. Centering of the normalized gene expression data was performed by subtracting the row means and scaling by dividing the columns by the standard deviation (SD) to generate a Z-score. Clinical data were taken from the supplementary table from the original paper. Response to ICB was based on RECIST criteria as described in the paper (Responders: CR/PR/SD, Non-Responders: PD).

Normalized gene expression data (Nanostring) and clinical data from patients treated with anti-CTLA-4 or anti-PD1 were taken from the supplementary data from Roh et al. ${ }^{40}$. Response to ICB was based on the classification from the Roh et al. manuscript (Responder or non-responder).

Heat maps were generated with matching genes between the STUB1-KO signature and external datasets. Samples were ordered based on the average expression of the signature (average Z-score per sample).

\section{GSEA}

GSEAPreranked was performed using the BROAD javaGSEA standalone version (http://www.broadinstitute.org/gsea/downloads.jsp). Gene ranking was performed using the $\log _{2}$-fold change in gene expression between D10 and SK-MEL-147 
776 melanoma cells expressing either sgCtrl or sgSTUB1 that were treated with MART-1

777 T cells for eight hours. The pre-ranked gene list was run with 1000 permutations.

778

779 Acknowledgements

780 We thank all members of the Peeper and Blank laboratories as well as of the Division

781 of Molecular Oncology and Immunology for constructive feedback and valuable input.

782 We thank R. Mezzadra, C. Sun, T. Schumacher as well as J. Staring and T.

783 Brummelkamp for sharing reagents and cell lines. Furthermore, we thank the flow

784 cytometry, proteomics and sequencing core facilities as well as the animal housing

785 facility of The Netherlands Cancer Institute for their support.

786

787 Author contributions

788 G.A., D.W.V. and D.S.P. conceptualized the project, G.A. and D.W.V. performed the 789 experiments and contributed equally to this work. O.B.B. and M.A. performed the 790 proteomic profiling experiments. O.K. performed bioinformatic analyses for 791 transcriptomic profiling. M.A.L., B.B. and J.B. carried out mouse experiments. D.D.A. 792 performed experiments. J.D.L. provided wildtype and mutant IFNGR1-ORF 793 constructs. M.A. and O.B.B. acknowledge support of the X-omics Initiative, part of the 794 NWO National Roadmap for Large-Scale Research Infrastructures. G.A., D.W.V. and 795 D.S.P. wrote the manuscript. D.S.P. supervised this study.

\section{Competing Financial Interest Statement}

798 D.S.P. is co-founder, shareholder and advisor of Immagene B.V.

799 M.A.L. is co-founder, shareholder and C.E.O. of Immagene B.V.

800 The other authors report no competing financial interests. 
804

805

806

807

808

809

810

811

812

813

814

815

816

817

818

819

820

821

822

823

824

825

826

827

828

829

830

831

832

833

834

835

836

837

\section{References}

1. Larkin, J. et al. Combined Nivolumab and Ipilimumab or Monotherapy in Untreated Melanoma. N. Engl. J. Med. 373, 23-34 (2015).

2. Wolchok, J. D. et al. Overall Survival with Combined Nivolumab and Ipilimumab in Advanced Melanoma. N. Engl. J. Med. (2017) doi:10.1056/nejmoa1709684.

3. Restifo, N. P. et al. Loss of functional beta2-microglobulin in metastatic melanomas from five patients receiving immunotherapy. J. Natl. Cancer Inst. (1996) doi:10.1093/jnci/88.2.100.

4. Zaretsky, J. M. et al. Mutations Associated with Acquired Resistance to PD-1 Blockade in Melanoma. N. Engl. J. Med. 375, 819-29 (2016).

5. Gao, J. et al. Loss of IFN-y Pathway Genes in Tumor Cells as a Mechanism of Resistance to Anti-CTLA-4 Therapy. Cell 167, 397-404.e9 (2016).

6. Kearney, C. J. et al. Tumor immune evasion arises through loss of TNF sensitivity. Sci. Immunol. (2018) doi:10.1126/sciimmunol.aar3451.

7. Sharma, P., Hu-Lieskovan, S., Wargo, J. A. \& Ribas, A. Primary, Adaptive, and Acquired Resistance to Cancer Immunotherapy. Cell (2017) doi:10.1016/j.cell.2017.01.017.

8. Snyder, A. et al. Genetic basis for clinical response to CTLA-4 blockade in melanoma. N. Engl. J. Med. (2014) doi:10.1056/NEJMoa1406498.

9. Shukla, S. A. et al. Cancer-Germline Antigen Expression Discriminates Clinical Outcome to CTLA-4 Blockade. Cell (2018) doi:10.1016/j.cell.2018.03.026.

10. Rizvi, N. a. et al. Mutational landscape determines sensitivity to PD-1 blockade in non-small cell lung cancer. Science (80-. ). 348, 124-8 (2015).

11. Van Allen, E. M. et al. Genomic correlates of response to CTLA-4 blockade in metastatic melanoma. Science (80-. ). (2015) doi:10.1126/science.aad0095.

12. Riaz, N. et al. Tumor and Microenvironment Evolution during Immunotherapy with Nivolumab. Cell (2017) doi:10.1016/j.cell.2017.09.028.

13. Topalian, S. L. et al. Safety, activity, and immune correlates of anti-PD-1 antibody in cancer. N. Engl. J. Med. (2012) doi:10.1056/NEJMoa1200690.

14. Rosenberg, J. E. et al. Atezolizumab in patients with locally advanced and metastatic urothelial carcinoma who have progressed following treatment with platinum-based chemotherapy: A single-arm, multicentre, phase 2 trial. Lancet (2016) doi:10.1016/S0140-6736(16)00561-4. 
15. Sade-Feldman, M. et al. Defining T Cell States Associated with Response to Checkpoint Immunotherapy in Melanoma. Cell (2018)

16. Tumeh, P. C. et al. PD-1 blockade induces responses by inhibiting adaptive immune resistance. Nature (2014) doi:10.1038/nature13954.

17. Ayers, M. et al. IFN-Y-related mRNA profile predicts clinical response to PD-1 blockade. J. Clin. Invest. (2017) doi:10.1172/JCI91190.

18. Shin, D. S. et al. Primary resistance to PD-1 blockade mediated by JAK1/2 mutations. Cancer Discov. 7, 188-201 (2017).

19. Vredevoogd, D. W. et al. Augmenting Immunotherapy Impact by Lowering Tumor TNF Cytotoxicity Threshold. Cell (2019) doi:10.1016/j.cell.2019.06.014.

20. Gooch, J. L., Herrera, R. E. \& Yee, D. The role of p21 in interferon y-mediated growth inhibition of human breast cancer cells. Cell Growth Differ. (2000).

21. Dai, C. \& Krantz, S. B. Interferon $y$ induces upregulation and activation of caspases 1,3 , and 8 to produce apoptosis in human erythroid progenitor cells. Blood (1999).

22. Siegmund, D. et al. Death Receptor-Induced Signaling Pathways Are Differentially Regulated by Gamma Interferon Upstream of Caspase 8 Processing. Mol. Cell. Biol. (2005) doi:10.1128/mcb.25.15.6363-6379.2005.

23. Fulda, S. \& Debatin, K. M. IFNy sensitizes for apoptosis by upregulating caspase-8 expression through the Stat1 pathway. Oncogene (2002) doi:10.1038/sj.onc.1205255.

24. Liu, D. et al. Integrative molecular and clinical modeling of clinical outcomes to PD1 blockade in patients with metastatic melanoma. Nat. Med. 25, (2019).

25. Kaplan, D. H. et al. Demonstration of an interferon $\mathrm{Y}$-dependent tumor surveillance system in immunocompetent mice. Proc. Natl. Acad. Sci. U. S. A. (1998) doi:10.1073/pnas.95.13.7556.

26. Manguso, R. T. et al. In vivo CRISPR screening identifies Ptpn2 as a cancer immunotherapy target. Nature (2017) doi:10.1038/nature23270.

27. Pan, D. et al. A major chromatin regulator determines resistance of tumor cells to T cell-mediated killing. Science (80-. ). (2018) doi:10.1126/science.aao1710.

28. Shalem, O. et al. Genome-scale CRISPR-Cas9 knockout screening in human cells. Science 343, 84-7 (2014).

29. Hart, T. et al. Evaluation and design of genome-wide CRISPR/SpCas9 
knockout screens. G3 Genes, Genomes, Genet. 7, 2719-2727 (2017).

30. $\mathrm{Li}, \mathrm{W}$. et al. MAGeCK enables robust identification of essential genes from genome-scale CRISPR/Cas9 knockout screens. Genome Biol. (2014) doi:10.1186/s13059-014-0554-4.

31. Huang, G. Biotinylation of Cell Surface Proteins. BIO-PROTOCOL (2012) doi:10.21769/bioprotoc. 170 .

32. Ballinger, C. A. et al. Identification of CHIP, a Novel Tetratricopeptide RepeatContaining Protein That Interacts with Heat Shock Proteins and Negatively Regulates Chaperone Functions. Mol. Cell. Biol. (1999) doi:10.1128/mcb.19.6.4535.

33. Jiang, J. et al. CHIP is a U-box-dependent E3 ubiquitin ligase: Identification of Hsc70 as a target for ubiquitylation. J. Biol. Chem. (2001) doi:10.1074/jbc.M101968200.

34. Demand, J., Alberti, S., Patterson, C. \& Höhfeld, J. Cooperation of a ubiquitin domain protein and an E3 ubiquitin ligase during chaperone/proteasome coupling. Curr. Biol. (2001) doi:10.1016/S0960-9822(01)00487-0.

35. Connell, P. et al. The co-chaperone CHIP regulates protein triage decisions mediated by heat-shock proteins. Nat. Cell Biol. (2001) doi:10.1038/35050618.

36. Meacham, G. C., Patterson, C., Zhang, W., Younger, J. M. \& Cyr, D. M. The Hsc70 co-chaperone CHIP targets immature CFTR for proteasomal degradation. Nat. Cell Biol. (2001) doi:10.1038/35050509.

37. Kaplan, D. H., Greenlund, A. C., Tanner, J. W., Shaw, A. S. \& Schreiber, R. D. Identification of an interferon-y receptor a chain sequence required for JAK-1 binding. J. Biol. Chem. (1996) doi:10.1074/jbc.271.1.9.

38. Usacheva, A., Kotenko, S., Witte, M. M. \& Colamonici, O. R. Two Distinct Domains Within the N-Terminal Region of Janus Kinase 1 Interact with Cytokine Receptors. J. Immunol. (2002) doi:10.4049/jimmunol.169.3.1302.

39. van der Wal, L. et al. Improvement of ubiquitylation site detection by Orbitrap mass spectrometry. J. Proteomics (2018) doi:10.1016/j.jprot.2017.10.014.

40. Roh, W. et al. Integrated molecular analysis of tumor biopsies on sequential CTLA-4 and PD-1 blockade reveals markers of response and resistance. Sci. Transl. Med. (2017) doi:10.1126/scitransImed.aah3560.

41. Dighe, A. S., Richards, E., Old, L. J. \& Schreiber, R. D. Enhanced in vivo growth and resistance to rejection of tumor cells expressing dominant negative 
906

907

908

909

910

911

912

913

914

915

916

917

918

919

920

921

922

923

924

925

926

927

928

929

930

931

932

933

934

935

936

937

938

939

IFNy receptors. Immunity (1994) doi:10.1016/1074-7613(94)90087-6.

42. Londino, J. D. et al. Post-translational modification of the interferon-gamma receptor alters its stability and signaling. Biochem. J. (2017) doi:10.1042/BCJ20170548.

43. Ferrao, R. et al. The Structural Basis for Class II Cytokine Receptor Recognition by JAK1. Structure (2016) doi:10.1016/j.str.2016.03.023.

44. Singh, S. et al. Loss of ELF5-FBXW7 stabilizes IFNGR1 to promote the growth and metastasis of triple-negative breast cancer through interferon- $y$ signalling. Nat. Cell Biol. (2020) doi:10.1038/s41556-020-0495-y.

45. Mezzadra, R. et al. Identification of CMTM6 and CMTM4 as PD-L1 protein regulators. Nature (2017) doi:10.1038/nature23669.

46. Boshuizen, J. \& Peeper, D. S. Rational Cancer Treatment Combinations: An Urgent Clinical Need. Molecular Cell (2020) doi:10.1016/j.molcel.2020.05.031.

47. Gomez-Eerland, R. et al. Manufacture of gene-modified human T-cells with a memory stem/central memory phenotype. Hum. Gene Ther. Methods 25, 27787 (2014).

48. Tyanova, S. et al. The Perseus computational platform for comprehensive analysis of (prote)omics data. Nature Methods (2016) doi:10.1038/nmeth.3901.

49. Brockmann, M. et al. Genetic wiring maps of single-cell protein states reveal an off-switch for GPCR signalling. Nature (2017) doi:10.1038/nature22376.

50. Cox, J. et al. Accurate proteome-wide label-free quantification by delayed normalization and maximal peptide ratio extraction, termed MaxLFQ. Mol. Cell. Proteomics (2014) doi:10.1074/mcp.M113.031591.

51. Trapnell, C., Pachter, L. \& Salzberg, S. L. TopHat: Discovering splice junctions with RNA-Seq. Bioinformatics (2009) doi:10.1093/bioinformatics/btp120.

52. Love, M. I., Huber, W. \& Anders, S. Moderated estimation of fold change and dispersion for RNA-seq data with DESeq2. Genome Biol. (2014) doi:10.1186/s13059-014-0550-8.

53. Dobin, A. et al. STAR: Ultrafast universal RNA-seq aligner. Bioinformatics (2013) doi:10.1093/bioinformatics/bts635.

54. Anders, S., Pyl, P. T. \& Huber, W. HTSeq-A Python framework to work with high-throughput sequencing data. Bioinformatics (2015) doi:10.1093/bioinformatics/btu638. 
Figure pioRxiv preprint doi: https://doi.org/10.1101/2020.07.07.191650; this version posted July 7,2020 . The copyright holder for this preprint

a

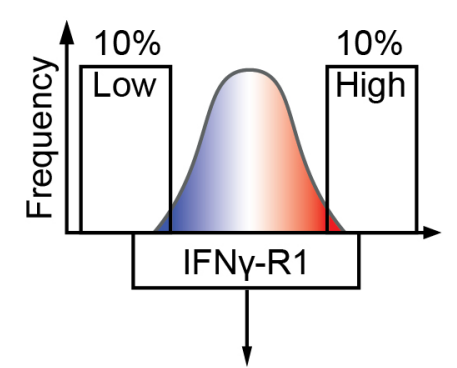

Sort by FACS

d

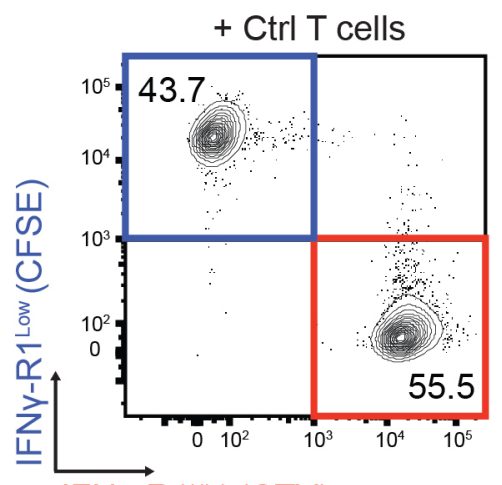

IFNy-R1 ${ }^{\text {High }}$ (CTV) w) is the author/funder, who has granted bioRxiv a license to display th
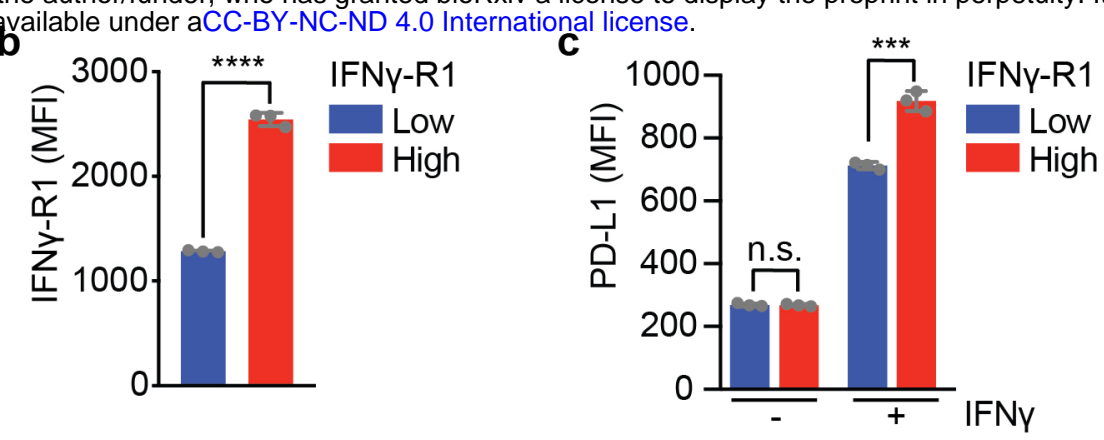

e
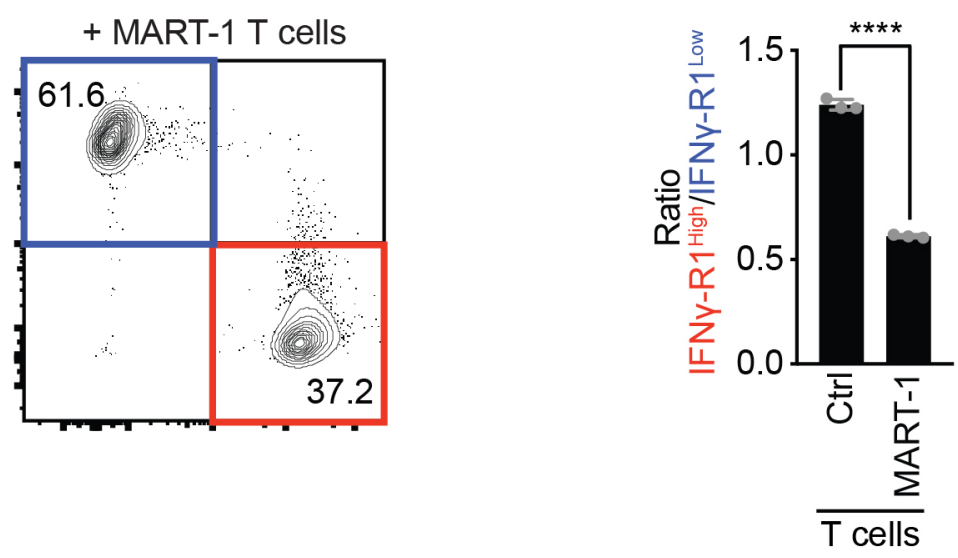

Genome-wide CRISPR/Cas9 sgRNA library
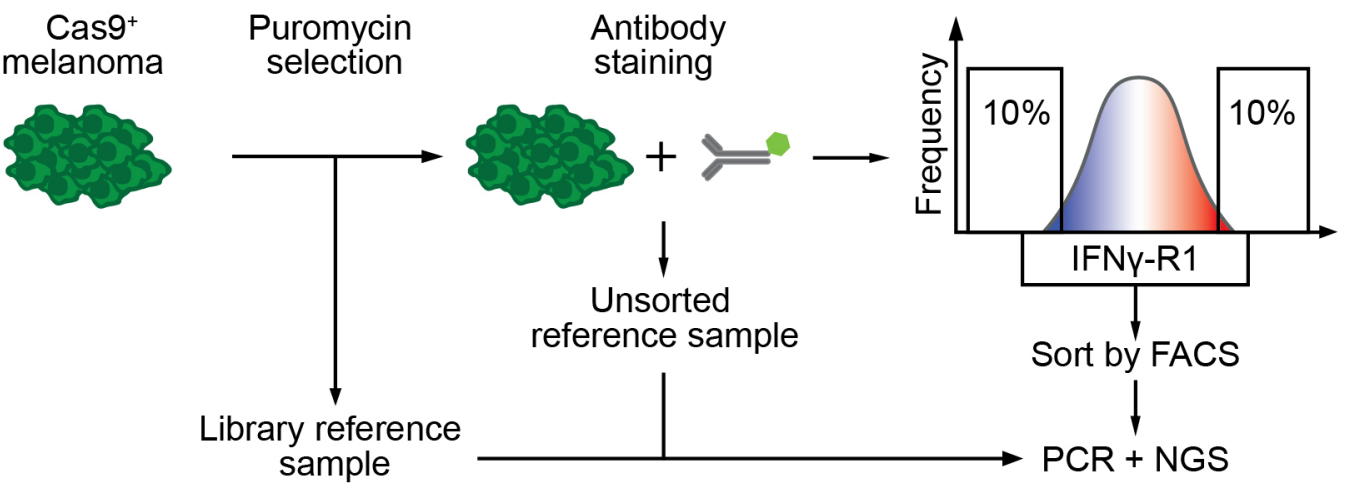

g

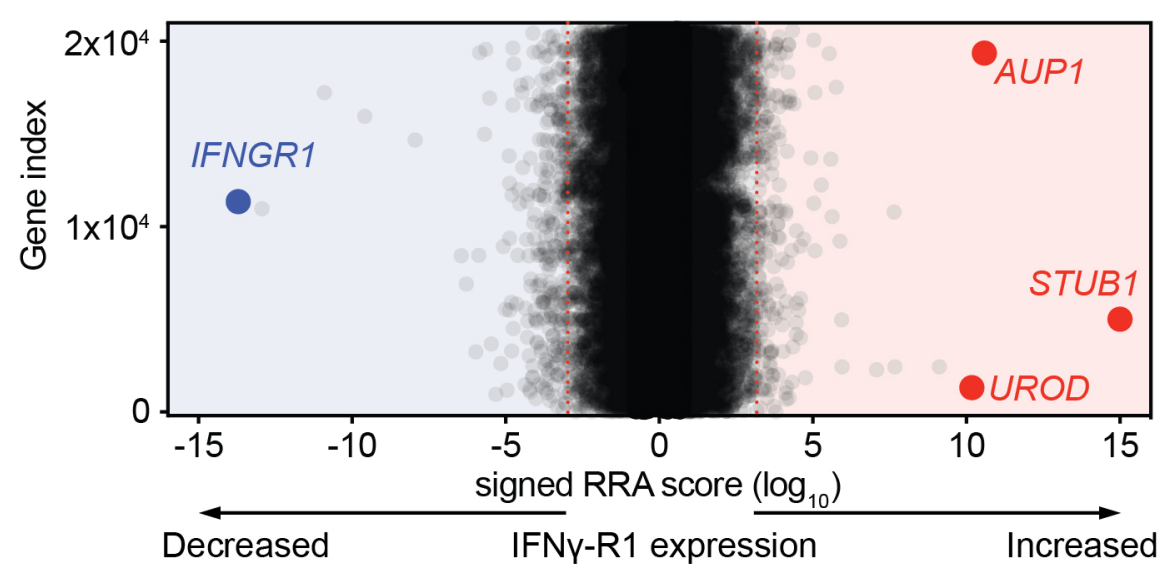

h

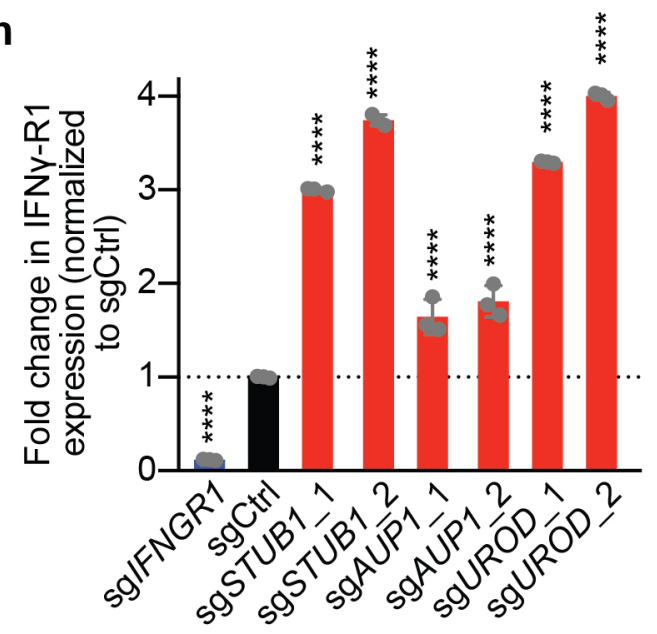


940 Main Figure Legends

941

942 Figure 1: Genome-wide CRISPR/Cas9 knockout screen identifies negative 943 regulators of IFNy-R1 expression to modulate its cell surface abundance.

944 a, Schematic outline of the FACsorting strategy to establish IFNy-R1 ${ }^{\text {High }}$ and IFNy945 R1 ${ }^{\text {Low }}$ D10 human melanoma cell populations.

946 b, Mean Fluorescence Intensity (MFI) of IFNY-R1 expression on D10 melanoma cells

947 two days after sorting the cells by flow cytometry into IFNY-R1 ${ }^{\text {High }}$ and IFNY-R1 $1^{\text {Low }}$ 948 subpopulations.

949 c, Assessment of IFNy-induced PD-L1 expression of IFNY-R1 ${ }^{\text {High }}$ and IFNy-R1 $1^{\text {Low }}$ 950 sorted cell populations 24 hours after treatment with $10 \mathrm{ng} / \mathrm{ml}$ IFNy.

951 d, Flow cytometry plot of the in vitro competition assay of IFNy-R $1^{\text {High }}$ vs. IFNy-R1 $1^{\text {Low }}$ 952 cells co-cultured with either MART-1 or Ctrl T cells.

953 e, Quantification of the ratio IFNy-R1 $1^{\text {High }}$ : IFNY-R1 ${ }^{\text {Low }}$ in competition assay of (d).

954 f, Schematic outline of the FACsort-based genome-wide CRISPR-KO screen to 955 identify genes regulating IFNY-R1 cell surface expression.

956 g, Screen results; red dotted lines indicate FDR cutoff $<0.25$ for genes enriched in $95710 \%$ of cells with the highest (right) or lowest (left) IFNy-R1 expression, as calculated 958 by MAGeCK analysis. Gene names indicate top enriched sgRNAs in cells with the $95910 \%$ highest IFNy-R1 expression (right), as well as the sgRNAs targeting IFNGR1 960 (left), serving as a positive control.

961 h, Quantification of IFNy-R1 expression by flow cytometry on cells expressing the 962 indicated sgRNAs, plotted as fold-change in IFNy-R1-MFI relative to sgCtrl-expressing 963 cells.

964 Mean \pm SD in (b), ${ }^{* * *} \mathrm{p}<0.0001$, unpaired t-test for three biological replicates.

965 Mean \pm SD in (c), ${ }^{* *} p=0.000467$, n.s. $p=0.806896$, unpaired t-test for three biological 966 replicates.

967 Mean \pm SD in $(\mathbf{e}):{ }^{* * *} p<0.0001$, unpaired t-test for three biological replicates.

968 Mean \pm SD in $(\mathbf{h}):{ }^{* * *} p<0.0001$, ordinary one-way ANOVA for three biological 969 replicates with Dunnett post hoc testing.

970 
Figure 2 bioRxiv preprint doi: https://doi.org/10.1101/2020.07.07.191650; this version posted July 7, 2020. The copyright holder for this preprint (which was not certified by peer review) is the author/funder, who has granted bioRxiv a license to display the preprint in perpetuity. It is

a

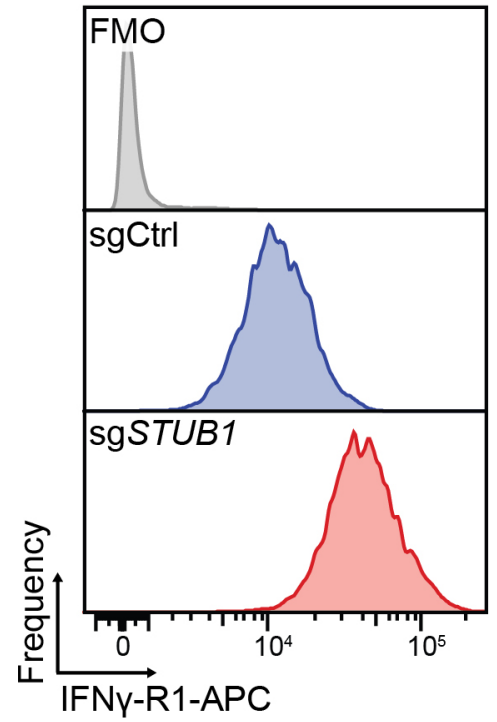
made ayailable under aCC-BY-NC-ND 4.0 Internakonal license.
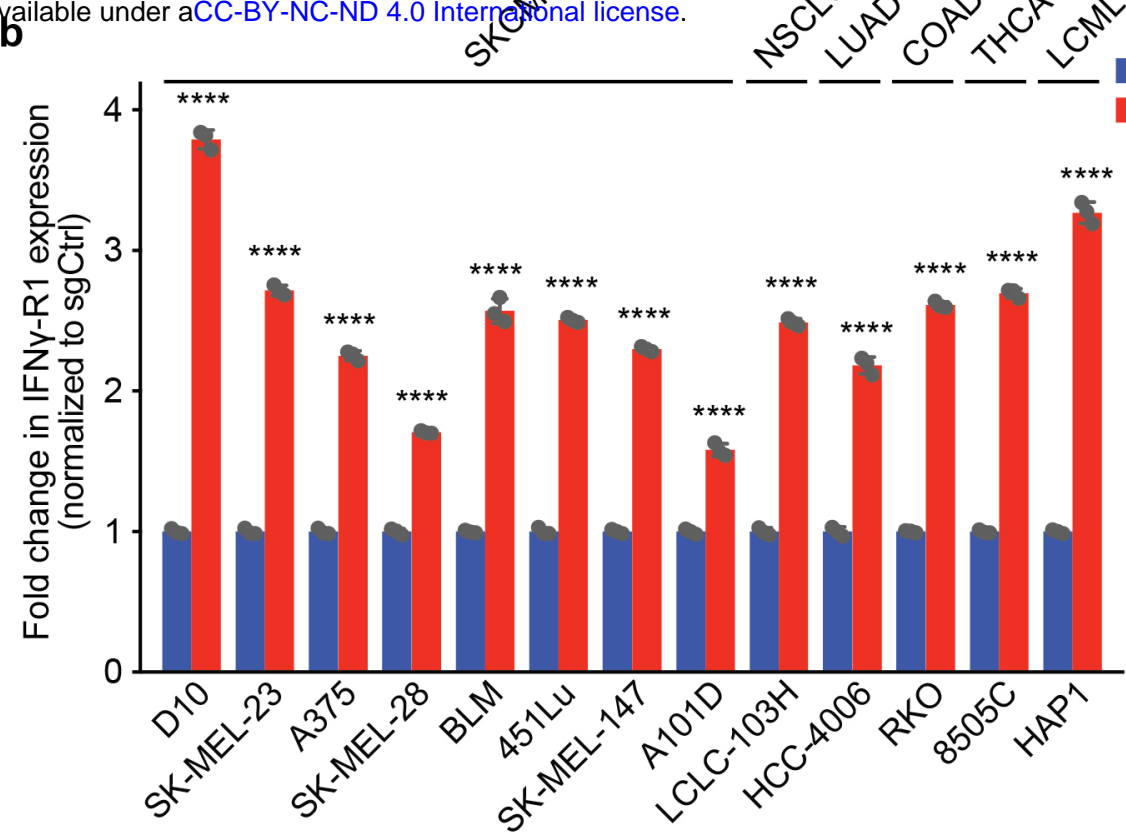

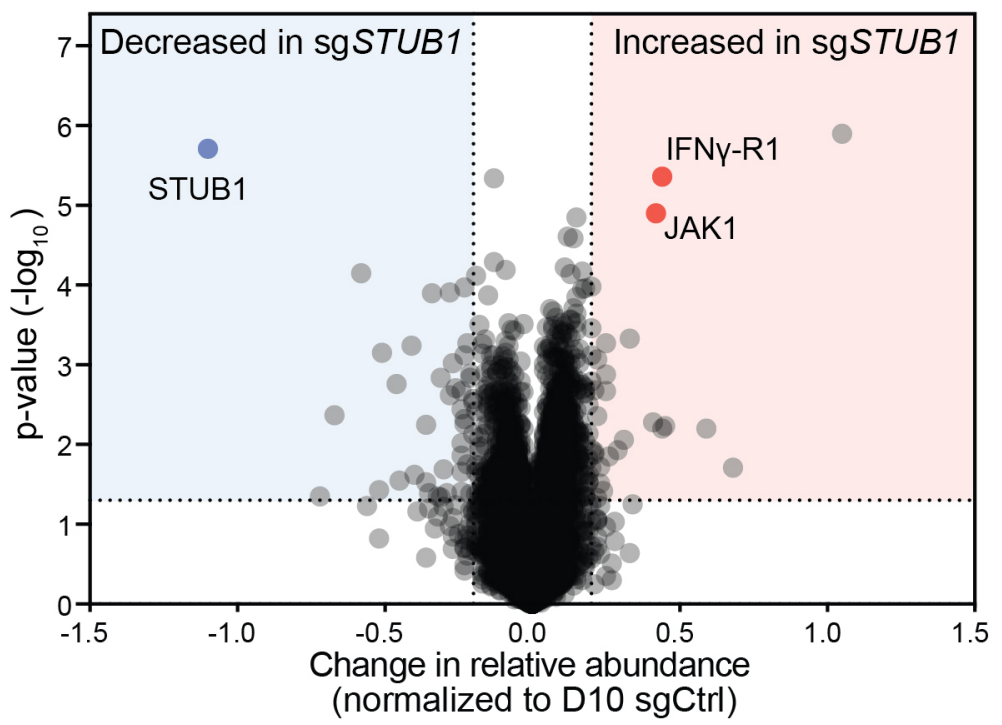

e

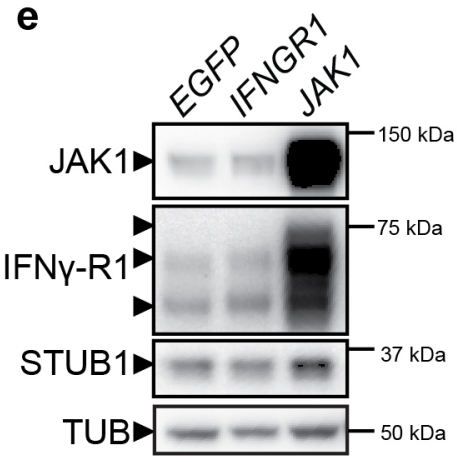

f

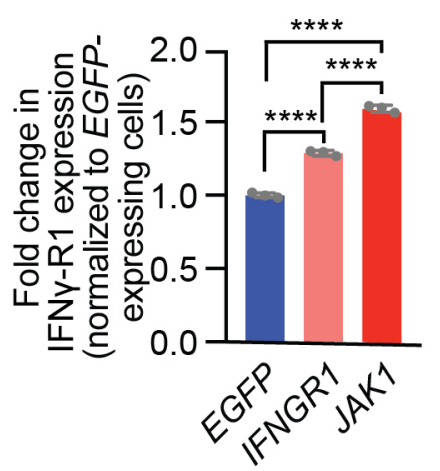

d

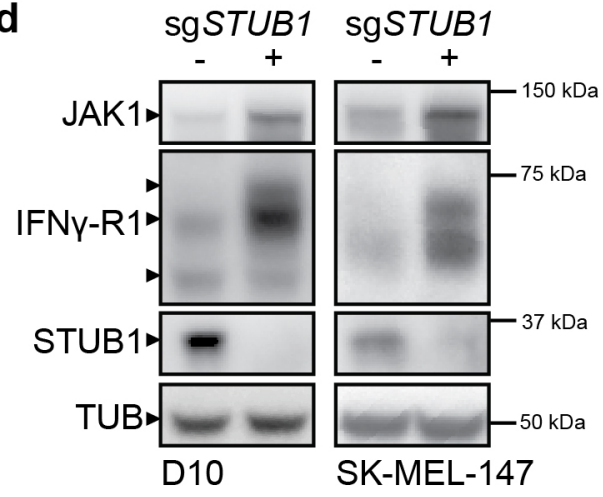
IFNGR1-KO

JAK1-KO

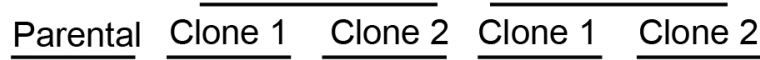
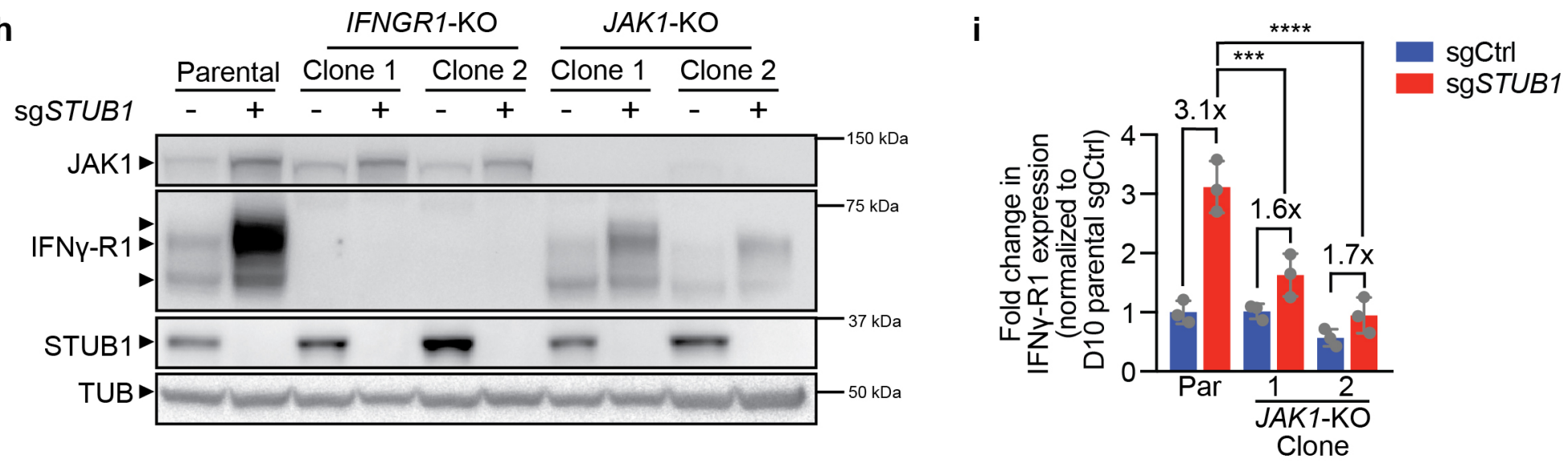
Figure 2: STUB1 destabilizes cell surface IFNY-R1 in JAK1-dependent and JAK1independent manners

974 a, Histograms of IFNy-R1 expression on D10 melanoma cells as measured by flow

975 cytometry in cells expressing the indicated sgRNAs. FMO: fluorescence minus one,

976 APC: Allophycocyanin.

977 b, Relative IFNy-R1 expression (normalized to each respective sgCtrl) measured by

978 flow cytometry in indicated human tumor cell lines expressing either sgCtrl or

979 sgSTUB1. Cancer types of the cell lines are abbreviated as follows: SKCM, skin

980 cutaneous melanoma; NSCLC, non-small-cell lung cancer; LUAD, lung

981 adenocarcinoma; COAD, colon adenocarcinoma; THCA, thyroid carcinoma.

982 c, Results of proteomic profiling of D10 melanoma cells expressing either sgCtrl or

983 sgSTUB1. Highlighted are the top differentially regulated proteins shared between

984 sgCtrl and sgSTUB1-expressing D10 and SK-MEL-147 cells (Supplementary Fig.

985 4e).

986 d, Immunoblot of D10 (left) and SK-MEL-147 (right) melanoma cells lines expressing

987 either sgCtrl or sgSTUB1. Whole cell lysates were immunoblotted for the indicated 988 proteins (TUB is tubulin).

989 e, Immunoblot of D10 melanoma cells ectopically expressing either EGFP (control),

990 IFNGR1 or JAK1. Whole cell lysates were immunoblotted for the indicated proteins

991 (TUB is tubulin).

992 f, Quantification of IFNY-R1 expression (relative to that in EGFP-expressing cells) by

993 flow cytometry in D10 melanoma cells ectopically expressing either EGFP (control),

994 IFNGR1 or JAK1.

$995 \mathbf{g}$, Results of qPCR analysis for the mRNA expression of IFNGR1 (relative to RPL13 996 expression) in D10 cells expressing either EGFP, IFNGR1 or JAK1. IFNGR1

997 expression was normalized to that in EGFP-expressing cells.

$998 \mathbf{h}$, Immunoblot of either parental D10 melanoma cells, D10 IFNGR1-KO clones or 999 JAK1-KO clones expressing either sgCtrl or sgSTUB1. Whole cell lysates were blotted 1000 for the indicated proteins (TUB is tubulin).

1001 i, Quantification of IFNy-R1 protein levels (relative to loading control and normalized

1002 to D10 parental sgCtrl-expressing cells) from (i).

1003 Mean $\pm S D$ in $(\mathbf{b}),{ }^{* * * *} p<0.0001$, multiple t-tests for three biological replicates,

1004 Mean \pm SD in $(\mathbf{f}):{ }^{* * * *} p<0.0001$, ordinary one-way ANOVA for three biological 1005 replicates with Tukey post hoc testing. 
1006 Mean \pm SD in $(\mathbf{g})$ : n.s. $p=0.8001,{ }^{* * * *} p<0.0001$, ordinary one-way ANOVA for three

1007 biological replicates with Dunnett's post hoc testing.

1008 Mean \pm SD in $(\mathbf{i}):{ }^{* * *} p=0.0004,{ }^{* * * *} p<0.0001$, ordinary one-way ANOVA for three

1009 immunoblots with Tukey post hoc testing.

1010

1011 
Figure 3 bioRxiv preprint doi: https://doi.org/10.1101/2020.07.07.191650; this version posted July 7, 2020. The copyright holder for this preprint (which was not certified by peer review) is the author/funder, who has granted bioRxiv a license to display the preprint in perpetuity. It is a sgCtrl sgSTUB1 madebvailable under aCC-BVENfficled 4.0 Interational license. Vehicle d

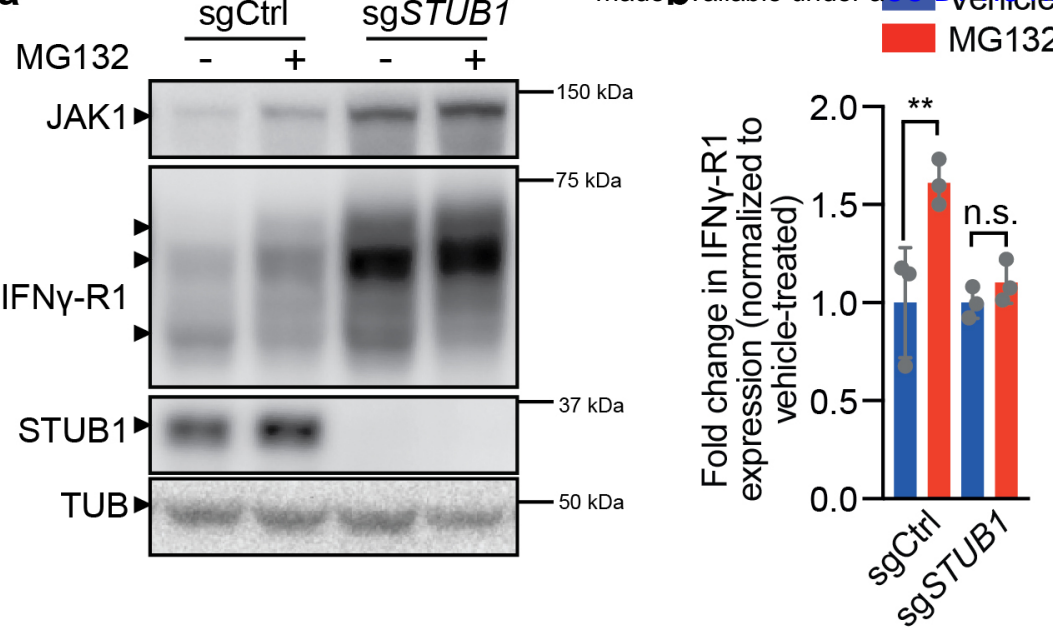

$\mathbf{f}$
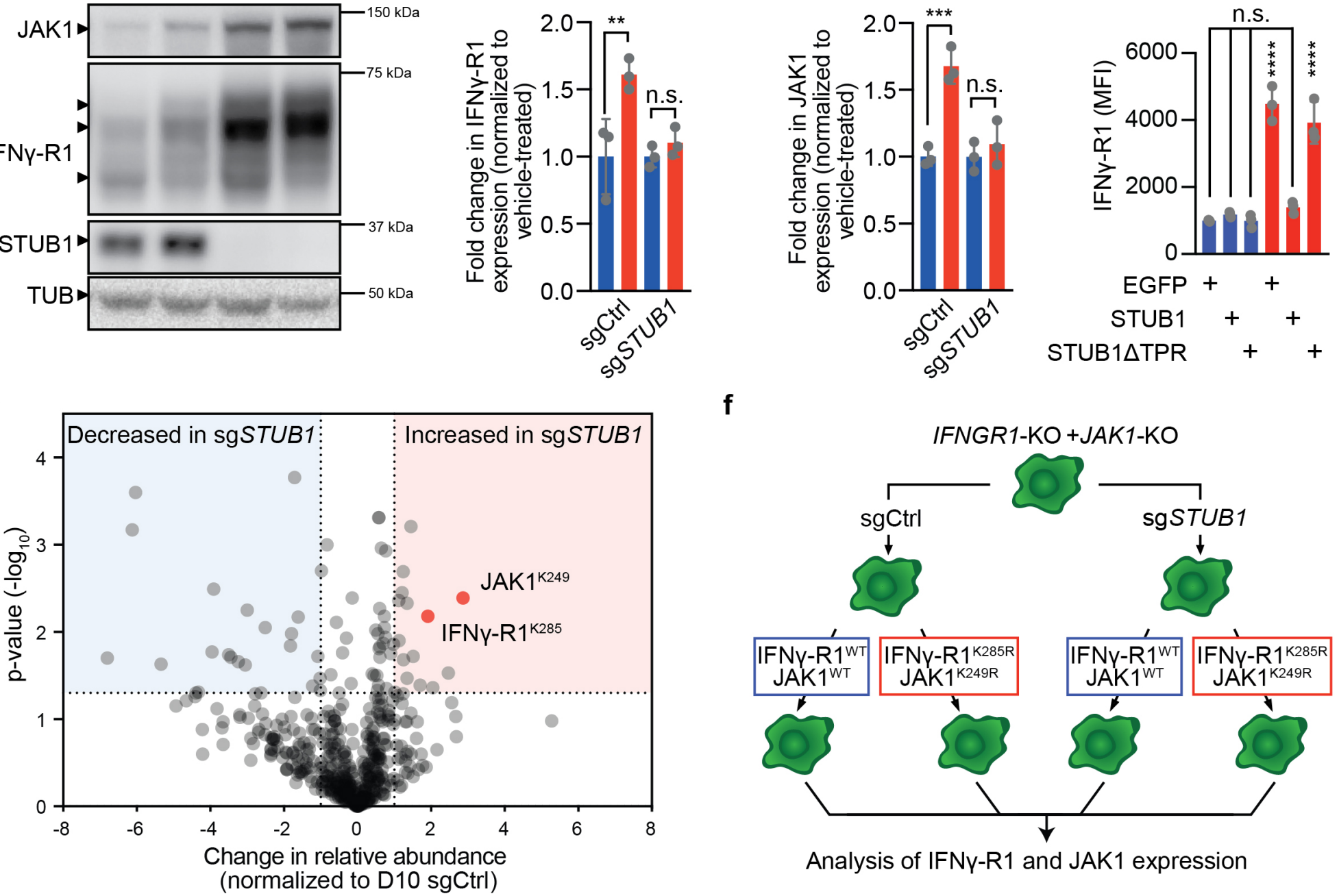

Analysis of IFNY-R1 and JAK1 expression

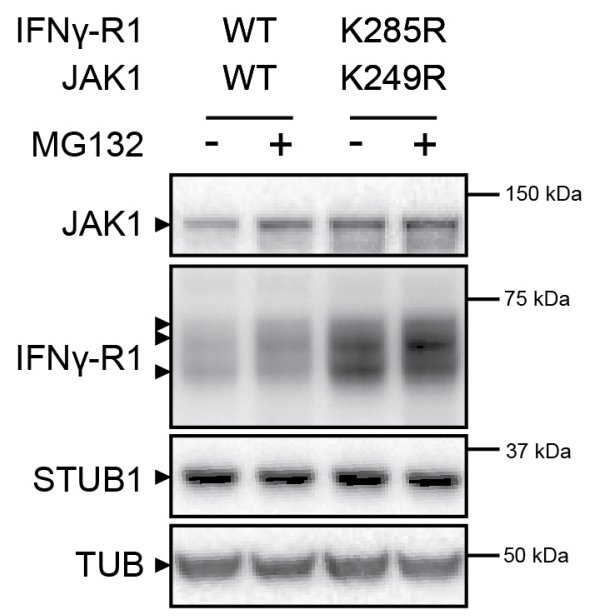

h

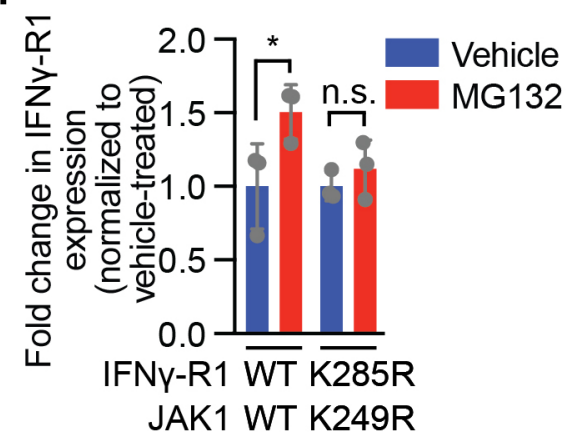

i

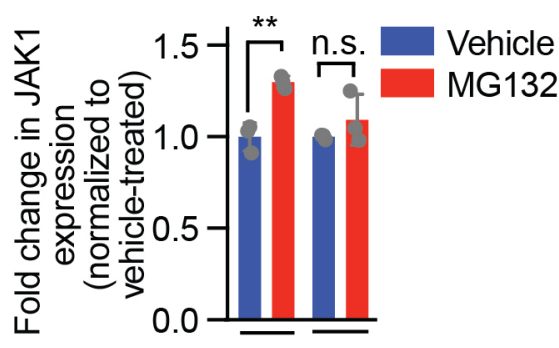

IFNY-R1 WT K285R

JAK1 WT K249R
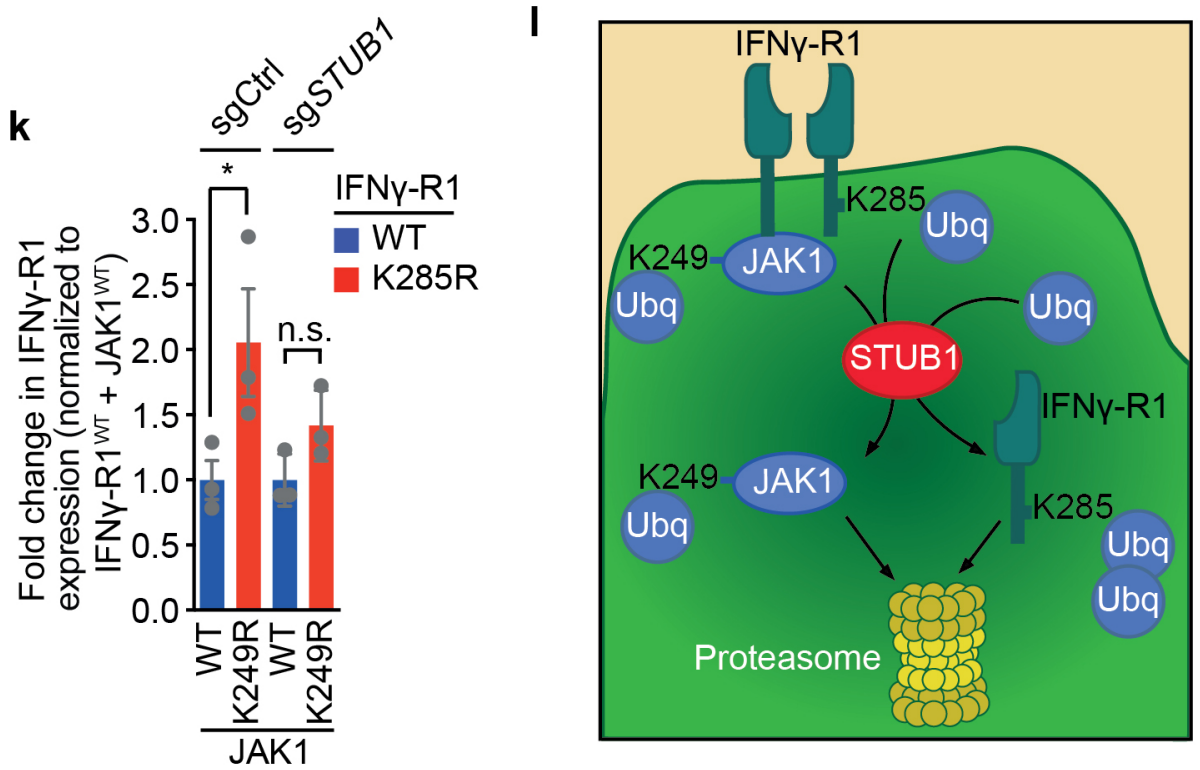

IFNY-R1 $\frac{\text { sgCtrl }}{W T \quad K 285 R} \frac{\text { sgSTUB1 }}{W T \quad K 285 R}$

JAK1 WT K249R WT K249R

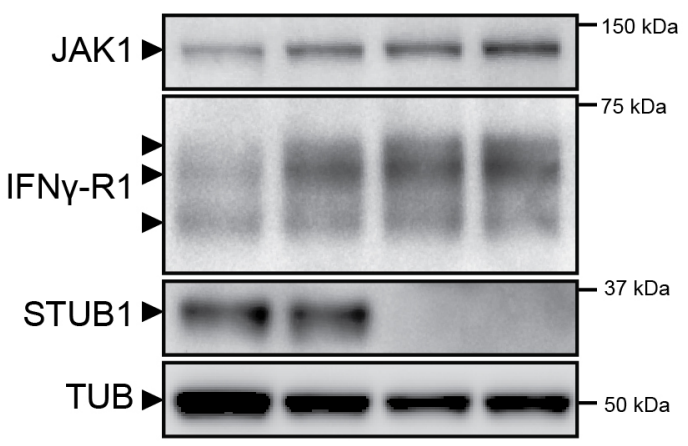


1012 Figure 3: STUB1 drives proteasomal degradation of IFNy receptor complex 1013 through IFNY-R1 ${ }^{\mathrm{K} 285}$ and JAK1 ${ }^{\mathrm{K} 249}$ residues.

1014 a, Immunoblot of D10 melanoma cells expressing either sgCtrl or sgSTUB1 treated 1015 with either vehicle or $10 \mu \mathrm{M}$ MG132 for four hours. Whole-cell lysates were 1016 immunoblotted for the indicated proteins (TUB is tubulin).

1017 b, Quantification of IFNY-R1 protein levels (relative to loading control and normalized 1018 to vehicle-treated group) from (a).

1019 c, Quantification of JAK1 protein levels (relative to loading control and normalized to 1020 vehicle-treated group) from (a).

1021 d, MFI of IFNY-R1 expression on D10 cells expressing sgCtrl or sgSTUB1, which 1022 ectopically express either 3xFLAG-tagged EGFP, full length STUB1 or STUB1 lacking $1023 \quad \mathrm{~N}$-terminal residues $1-72$ of the TPR domain.

1024 e, Relative change in K-epsilon-diglycine motif-containing peptides in sgSTUB11025 expressing cells, normalized to sgCtrl-expressing cells. Highlighted are peptides that 1026 also exhibit significant differential regulation at total protein level as assessed by global 1027 proteomic analysis (Fig. 2c and Supplementary Figure 2e)

$1028 \mathrm{f}$, Schematic image depicting the reconstitution of either IFNY-R1 ${ }^{\mathrm{WT}}$ and JAK1 ${ }^{\mathrm{WT}}$ or 1029 IFNY-R1 1285R and JAK1 1 $249 R$ ORFs in IFNGR1-KO + JAK1-KO D10 melanoma clones 1030 in either sgCtrl- or sgSTUB1-expressing genetic background.

1031 g, Immunoblot of IFNGR1-KO + JAK1-KO D10 melanoma clones, reconstituted with 1032 either IFNY-R1 ${ }^{\mathrm{WT}}$ and JAK1WT or IFNy-R1 ${ }^{\mathrm{K} 285 \mathrm{R}}$ and JAK1 ${ }^{\mathrm{K} 249 \mathrm{R}}$ ORFs. The cells were 1033 subsequently treated with $10 \mu \mathrm{M}$ MG132 for four hours. Whole-cell lysates were 1034 immunoblotted for the indicated proteins (TUB is tubulin).

$1035 \mathbf{h}$, Quantification of IFNy-R1 protein levels (relative to loading control and normalized 1036 to vehicle-treated group) from $(\mathbf{g})$.

1037 i, Quantification of JAK1 protein levels (relative to loading control and normalized to 1038 vehicle-treated group) from $(\mathbf{g})$.

$1039 \mathrm{j}$, Immunoblot on whole cell lysates of IFNGR1-KO + JAK1-KO D10 melanoma clones 1040 reconstituted with the indicated IFNGR1 and/or JAK1 cDNAs, as outlined in (f). Whole 1041 cell lysates were immunoblotted for the indicated proteins (TUB is tubulin).

$1042 \mathbf{k}$, Fold change of IFNY-R1 MFI (relative to IFNGR1-WT+JAK1-WT-expressing cells) 1043 in IFNGR1-KO + JAK1-KO D10 melanoma clones reconstituted with the indicated 1044 IFNGR1 and JAK1 cDNAs, as outlined in (f). Bar chart represents an excerpt from 1045 Supplementary Fig. 3i. 
1046 I, Model of STUB1-mediated proteasomal degradation of IFNY-R1 and JAK1.

1047 Mean $\pm S D$ in $(\mathbf{b}),{ }^{* *} p=0.0085$, n.s. $p=0.8675$, ordinary one-way ANOVA for three

1048 biological replicates with Tukey post hoc testing.

1049 Mean \pm SD in (c), ${ }^{* * *} p=0.0007$, n.s. $p=0.7936$, ordinary one-way ANOVA for three

1050 biological replicates with Tukey post hoc testing.

1051 Mean $\pm S D$ in $(\mathbf{d}),{ }^{* * *} p<0.0001$, n.s. $p=0.7282$, n.s. $p=0.966$, n.s. $p=0.7154$, ordinary

1052 one-way ANOVA for three biological replicates with Tukey post hoc testing.

1053 Mean $\pm S D$ in $(\mathbf{h}),{ }^{*} p=0.0322$, n.s. $p=0.7414$, ordinary one-way ANOVA for three

1054 biological replicates with Sidak post hoc testing.

1055 Mean $\pm S D$ in $(i),{ }^{* *} p=0.0041$, n.s. $p=0.3570$, ordinary one-way ANOVA for three

1056 biological replicates with Sidak post hoc testing.

1057 Mean \pm SD in $(\mathbf{k}),{ }^{*} p=0.036$, n.s. $p=0.9812$, ordinary one-way ANOVA for three

1058 biological replicates with Tukey post hoc testing. 
a

sgCtrl

+IFNy $[\mathrm{ng} / \mathrm{ml}]$
sgSTUB1

+ IFNy [ng/ml]

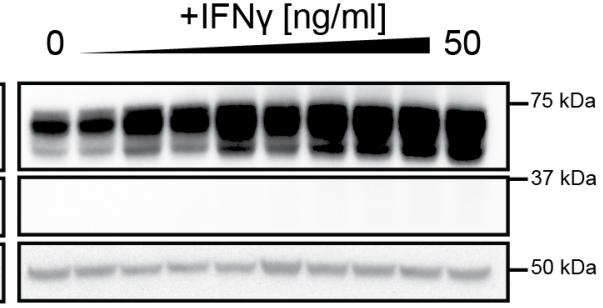

d $\begin{array}{ll}\text { MART-1 } 1^{+} \text {HLA-A2 } & \text { MART-1 T cells } \\ \text { sgCtrl or sgSTUB1 } & \text { or Ctrl T cells }\end{array}$
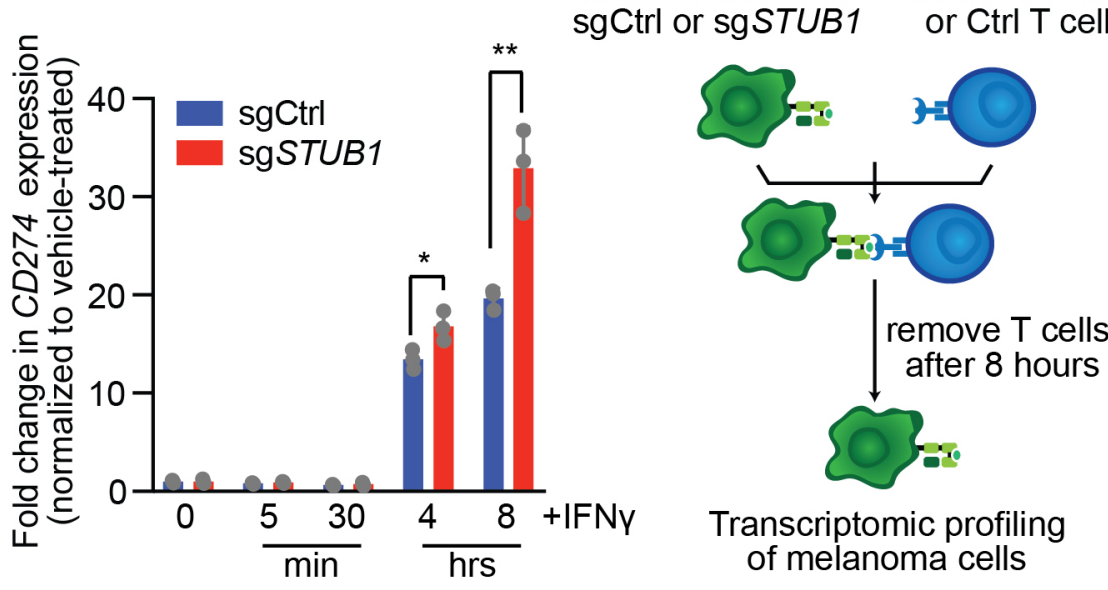

Transcriptomic profiling of melanoma cells

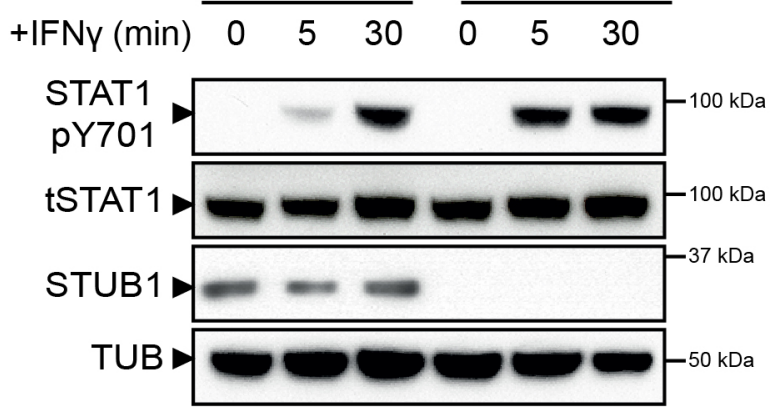

e HALLMARK

INTERFERON_GAMMA_RESPONSE $F D R=0.025$, NES $=1.57$

PID_TNF_PATHWAY $F D R=1, N E S=0.64$

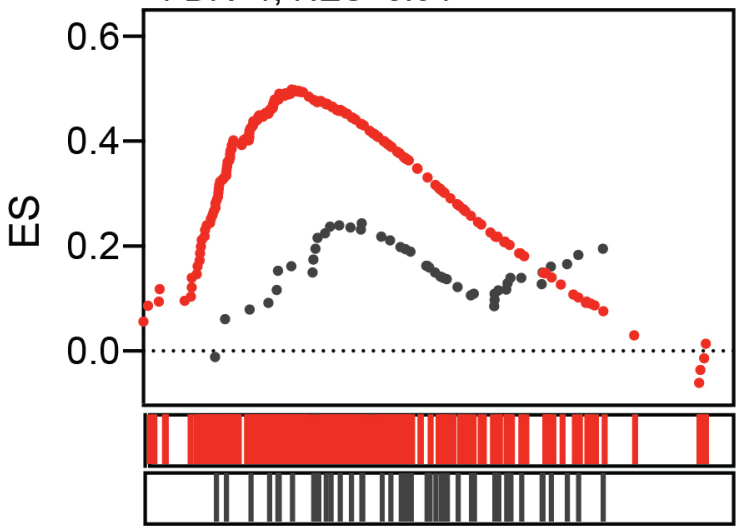

Upregulated f

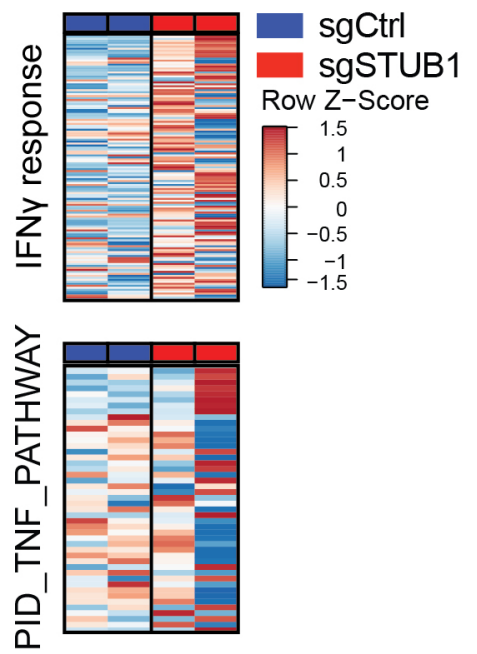

g

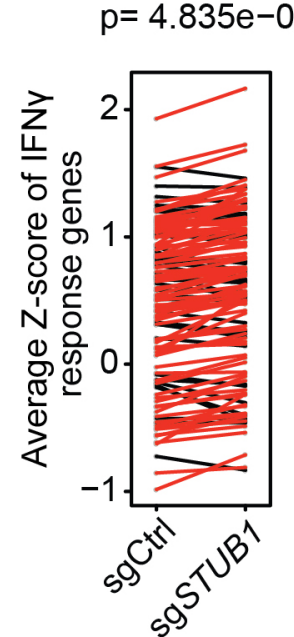

k

j sgSTUB1
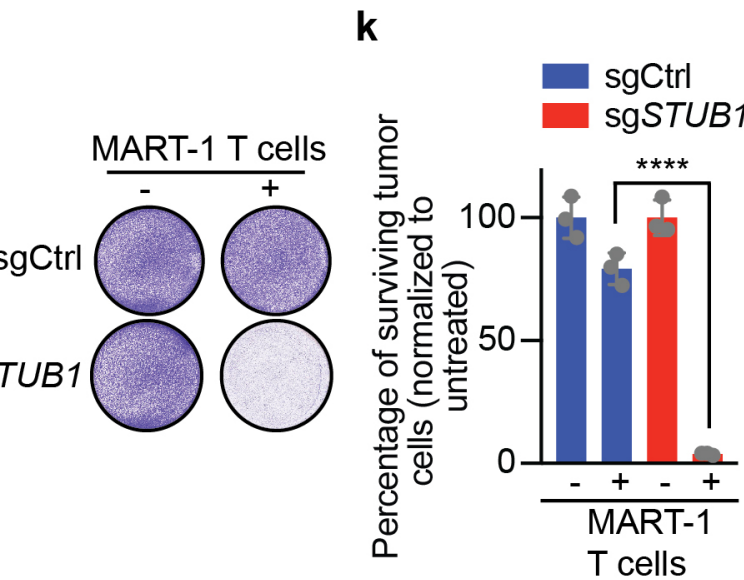

I

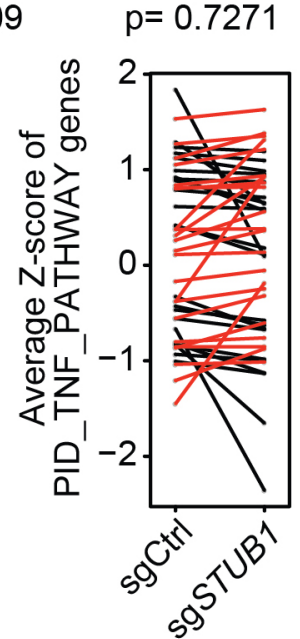

h

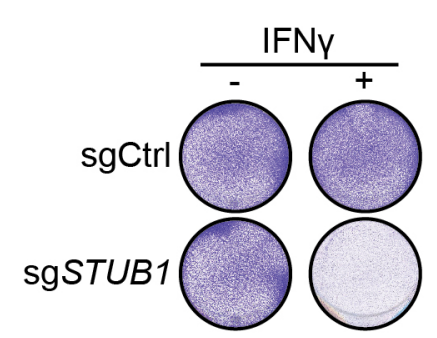

$-T$ cells + MART-1 T cells

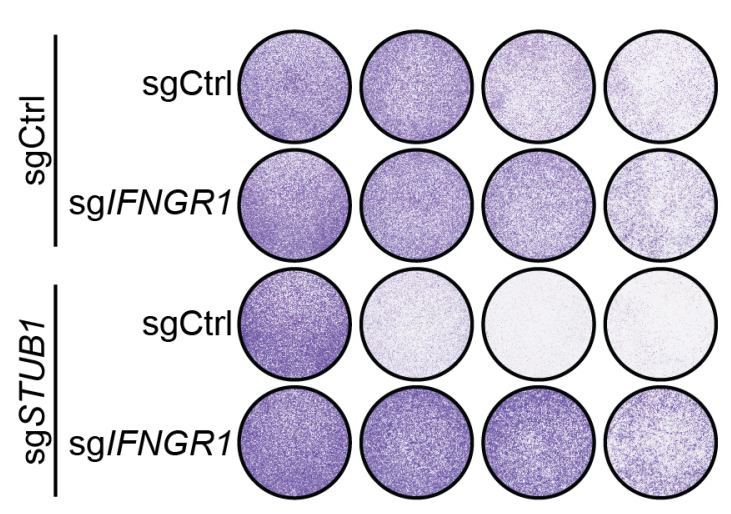

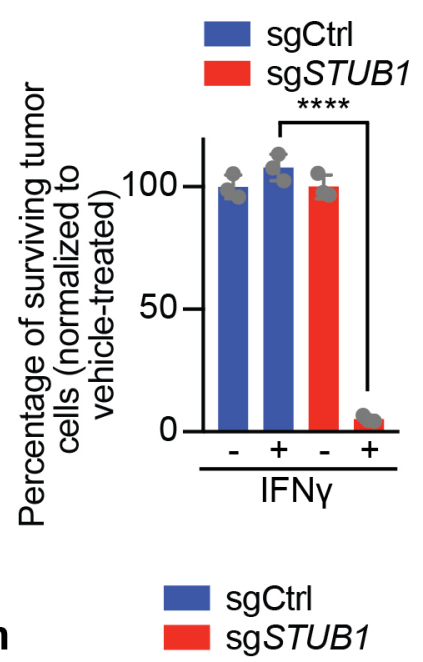

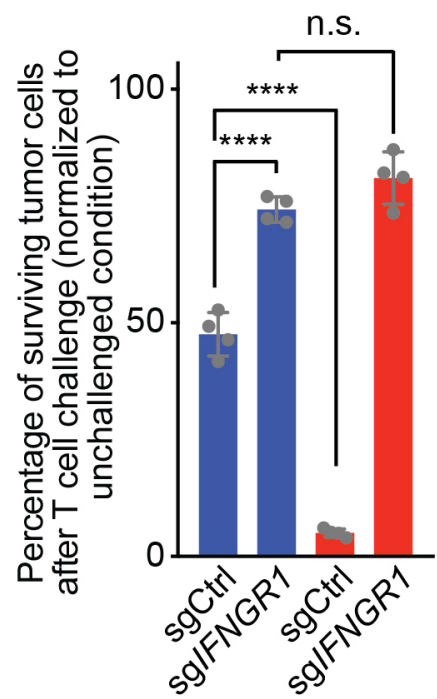


1060 Figure 4: STUB1 inactivation sensitizes melanoma cells to cytotoxic T cells 1061 through amplified IFNy signaling.

1062 a, Immunoblots of D10 melanoma cells expressing sgCtrl or sgSTUB1, treated with a 1063 two-fold serial dilution of IFNy (starting at $50 \mathrm{ng} / \mathrm{ml}$ ) for 30 minutes. Same protein 1064 amounts were loaded on two separate gels and whole cell lysates were immunoblotted 1065 for the indicated proteins (TUB is tubulin) and developed at the same time. The same 1066 exposure for the blots is shown.

1067 b, Immunoblot of D10 melanoma cells expressing sgCtrl or sgSTUB1, treated with 1068 either vehicle or $50 \mathrm{ng} / \mathrm{ml}$ IFNy for the indicated duration. Whole cell lysates were 1069 immunoblotted for phosphorylated-tyrosine-701 (pY701) of STAT1, total STAT1 1070 (tSTAT1), STUB1 and Tubulin (TUB).

1071 c, qPCR analysis for the mRNA expression of CD274 (encoding PD-L1) in D10 1072 melanoma cells expressing either sgCtrl or sgSTUB1, after treatment with $25 \mathrm{ng} / \mathrm{ml}$ 1073 IFNy for the indicated duration.

$1074 \mathrm{~d}$, Schematic depiction of the experimental setup to profile the transcriptomes of D10 and SK-MEL-147 melanoma cells expressing sgCtrl or sgSTUB1, which were co1076 cultured with either Ctrl or MART-1 T cells for eight hours.

1077 e, Gene set enrichment analysis on RNA sequencing results for D10 and SK-MEL1078147 melanoma cells co-cultured with MART-1 T cells for eight hours (from d).

1079 f, Differential gene expression analysis of IFNy response genes (derived by treating 1080 D10 and SK-MEL-147 melanoma cells with IFNy for eight hours, depicted in 1081 Supplementary Fig. 4d) and PID_TNF_PATHWAY genes in D10 melanoma cells co1082 cultured with MART-1 T cells for eight hours.

$1083 \mathbf{g}$, Difference in either IFNy response gene expression or expression of 1084 PID_TNF_PATHWAY genes between sgCtrl and sgSTUB1-expressing D10 1085 melanoma cells following MART-1 T cell challenge for eight hours.

$1086 \mathbf{h}$, Colony formation assay of D10 melanoma cells expressing sgCtrl or sgSTUB1 1087 treated with either vehicle or $3 \mathrm{ng} / \mathrm{ml}$ IFNy for five days.

1088 i, Quantification of colony formation assay shown in (h).

$1089 \mathrm{j}$, Colony formation assay of D10 melanoma cells expressing sgCtrl or sgSTUB1 1090 treated with either no or MART-1 T cells for 24 hours and subsequent culture for four 1091 days.

1092 k, Quantification of colony formation assay shown in (j). 
1093 I, Colony formation assay of D10 melanoma cells expressing the indicated sgRNAs,

1094 which were co-cultured with either no or MART-1 T cells at T cell : melanoma cell

1095 ratios 1:16, 1:8 and 1:4 (left to right) for 24 hours and stained four days later.

$1096 \mathbf{m}$, Quantification from (I) at a T cell : melanoma cell ratio of 1:8.

1097 Mean \pm SD in (c), ${ }^{* *} p=0.0064,{ }^{*} p=0.033$, multiple t-tests for three biological replicates.

1098 Average Z-score of respective genes in (g) from two biological replicates with paired 1099 t-test.

1100 Mean \pm SD in $(\mathbf{i}),{ }^{* * * *} p<0.0001$, ordinary one-way ANOVA for three biological replicates

1101 with Tukey post hoc testing.

1102 Mean $\pm S D$ in $(\mathbf{k}),{ }^{* * * *} p<0.0001$, ordinary one-way ANOVA for three biological 1103 replicates with Tukey post hoc testing.

1104 Mean \pm SD in $(m),{ }^{* * *} p<0.0001$, n.s. $p=0.1226$, ordinary one-way ANOVA for four 1105 biological replicates with Tukey post hoc testing.

1106 


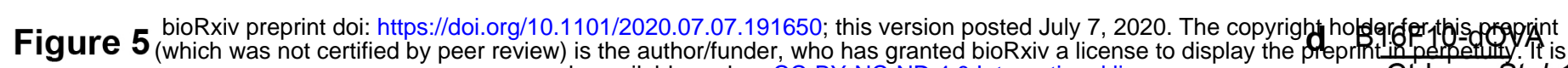
a made available under aCC-BY-NC-ND 4.0 International license.

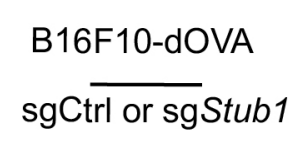

sgctritersgstubr

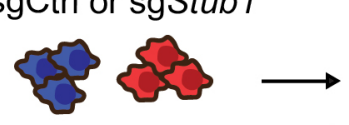

Immune-proficient

b
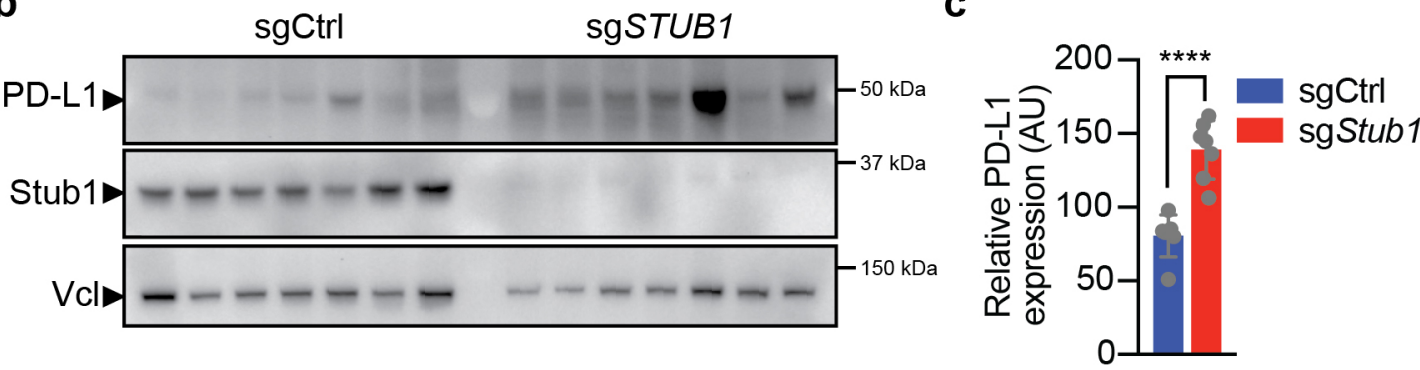

sgCtrl sgstub1

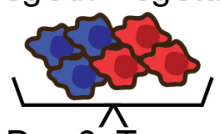

Day 0: Tumor

transplantation

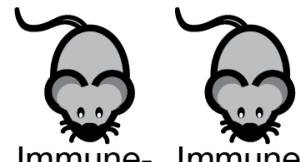

Immune- Immunedeficient proficient

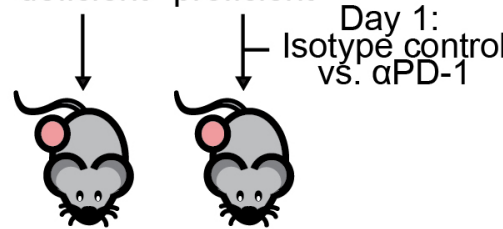

Day 12: Harvest tumor + assess tumor composition by flow cytometry e

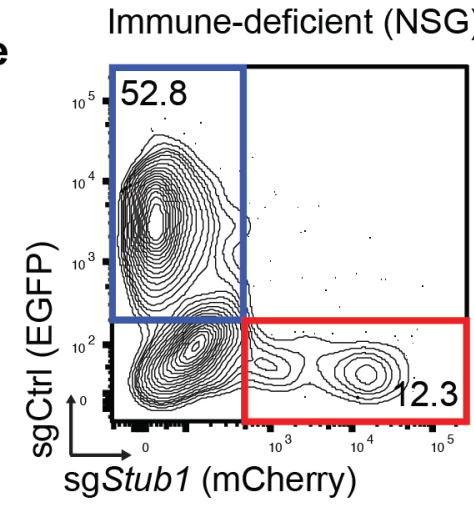

g

STUB1-KO signature expression

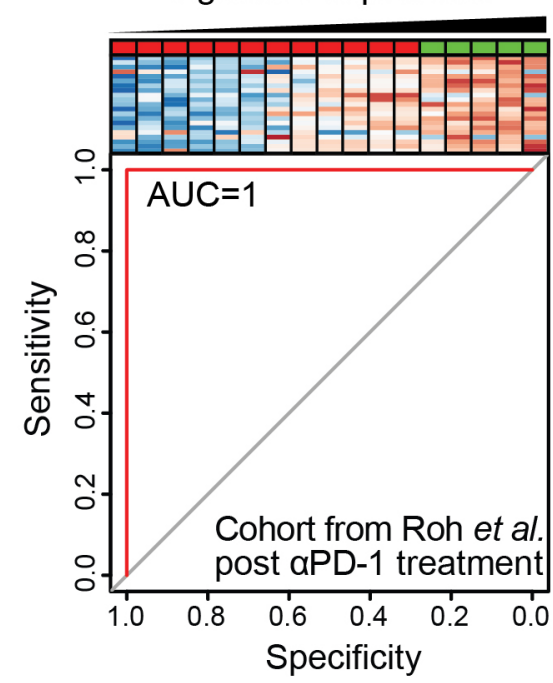

STUB1-KO

signature expression
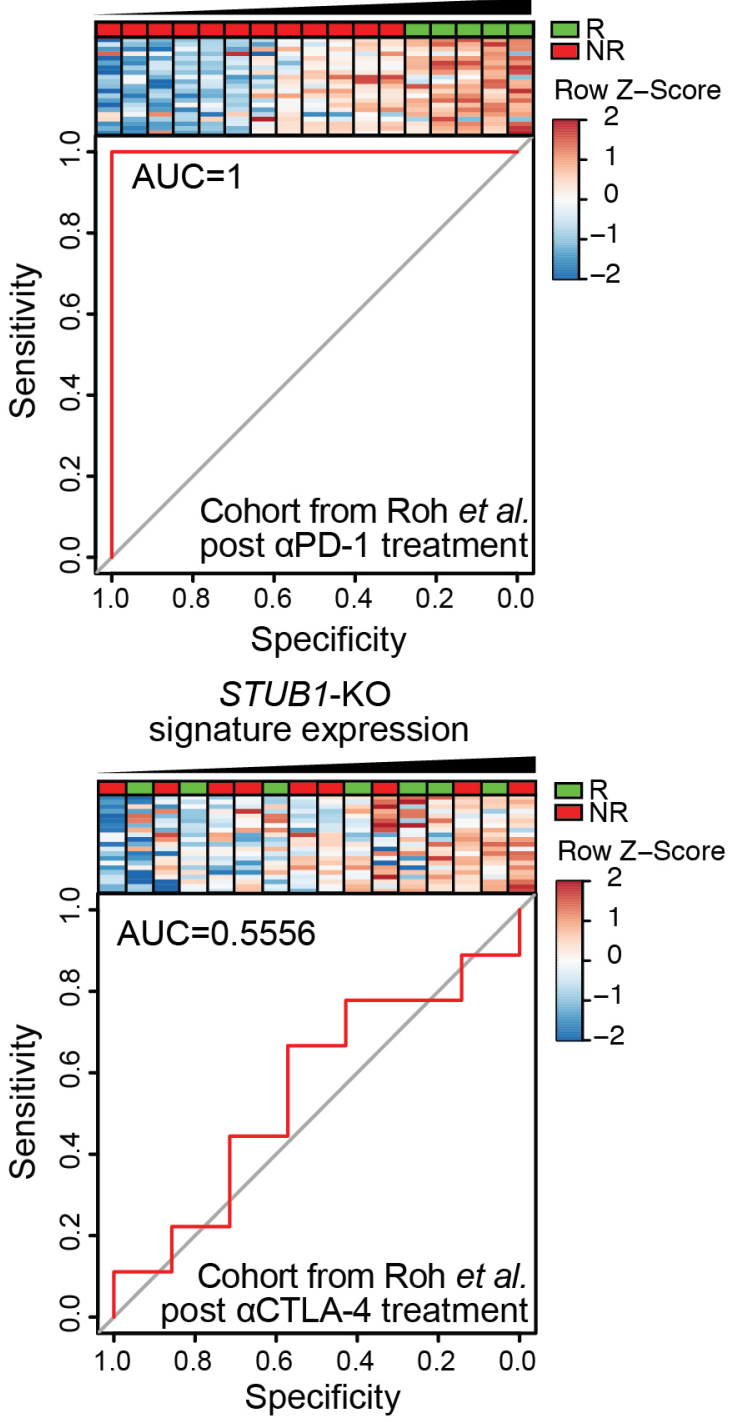

Immune-proficient

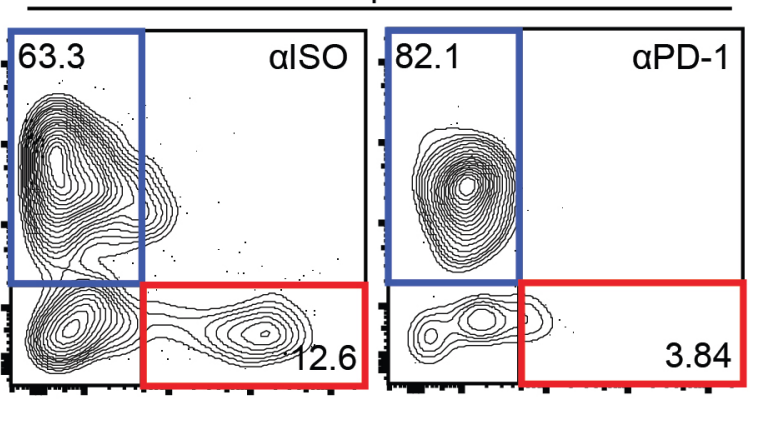

h

aPD-1 treatment

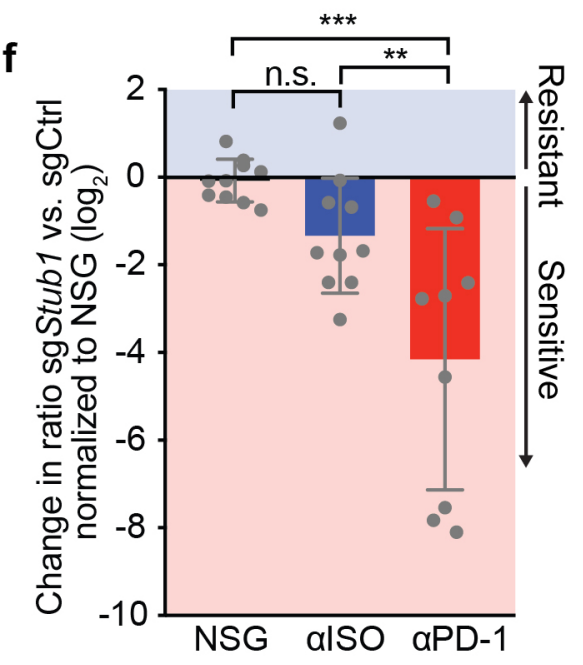

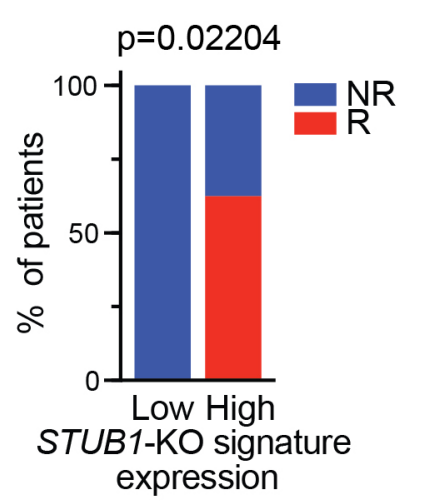

j

aCTLA-4 treatment

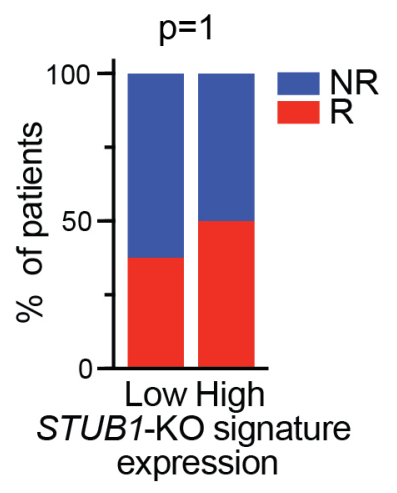


1108 Figure 5: STUB1 inactivation and anti-PD-1 treatment constitute a rational 1109 combination therapy approach.

1110 a, Experimental outline to assess PD-L1 expression on either sgCtrl or sgStub1 1111 tumors.

1112 b, Immunoblot of B16F10-dOVA in vivo tumor samples expressing either sgCtrl or 1113 sgStub1 (outlined in a) for the indicated proteins ( $\mathrm{Vcl}$ is vinculin).

1114 c, Quantification of PD-L1 protein levels (relative to loading control) of tumor samples 1115 from immunoblot shown in (b), AU=arbitrary units.

$1116 \mathbf{d}$, Schematic depiction of the in vivo competition assay modelling anti-PD-1 response 1117 with B16F10-dOVA cells expressing either sgCtrl or sgStub1, which were differentially 1118 labelled with EGFP and mCherry, respectively.

1119 e, Flow cytometry plots from each group of the in vivo experiment outlined in (d) NSG, 1120 Isotype control-treated (aISO), anti-PD-1-treated (aPD-1).

1121 f, Quantification of in vivo competition assay outlined in (d). Ratios of mCherry vs. 1122 EGFP were normalized to the NSG condition.

$1123 \mathbf{g}$, Composite plot consisting of a heat map showing melanoma patients from the post 1124 anti-PD-1 treatment cohort of Roh et al. 2017, sorted according to the STUB1-KO 1125 signature expression (average Z-score per sample), and ROC plot showing the 1126 predictive power of the STUB1-KO signature in this cohort.

$1127 \mathbf{h}$, Median of STUB1-KO signature expression in patients from (g) was used to divide 1128 patients into STUB1-KO signature high and low-expressing groups and percentage 1129 responders and non-responders in each group was plotted.

1130 i, Composite plot consisting of a heat map showing melanoma patients from the post 1131 anti-CTLA-4 treatment cohort of Roh et al. 2017, which were sorted according to 1132 STUB1-KO signature expression (average Z-score per sample) and ROC plot showing 1133 the predictive power of the STUB1-KO signature in this cohort.

$1134 \mathbf{j}$, Median of STUB1-KO signature expression in patients from (i) was used to divide 1135 patients into STUB1-KO signature high and low-expressing groups and percentage 1136 responders and non-responders in each group was plotted.

1137 Mean $\pm S D$ in (c), ${ }^{* * * *} p<0.0001$, unpaired two-tailed t-test, $n=7$ tumors per group.

1138 Mean $\pm S D$ in (f), ${ }^{* * *} p=0.0002,{ }^{* *} p=0.0073$, n.s. $p=0.2985$, ordinary one-way ANOVA 1139 with Tukey post hoc testing for $n=10$ in NSG and alSO and $n=9$ in aPD-1. 


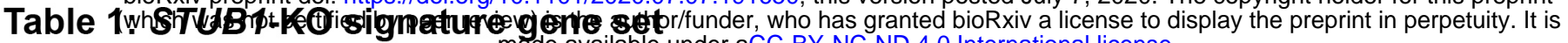

\begin{tabular}{|c|c|c|c|c|}
\hline$A C O D 1$ & EFNA1 & $I F I H 1$ & $P R D X 1$ & SNTB2 \\
\hline BIRC3 & FST & IFIT2 & PSMB8 & socs3 \\
\hline CCL2 & GBP1 & IFIT3 & $R A L A$ & SOD2 \\
\hline$C D 47$ & GBP2 & $I K Z F 2$ & $R C 3 H 1$ & THBS1 \\
\hline $\mathrm{CFH}$ & GBP4 & KYNU & RNF145 & TRIM22 \\
\hline CTSS & GBP5 & LIFR & SAMD9L & XRN1 \\
\hline CXCL10 & HEG1 & OAS2 & SAMHD1 & \\
\hline CXCL11 & $H L A-B$ & OSMR & SELENOK & \\
\hline CXCL9 & IDO1 & PARP14 & SFT2D2 & \\
\hline$D D \times 58$ & IFI44L & PLEKHS1 & SLAMF8 & \\
\hline
\end{tabular}




\section{Table 1: STUB1-KO signature gene set}

1143 STUB1-KO signature gene set established through transcriptomic profiling sgCtrl or

1144 sgSTUB1-expressing D10 and SK-MEL-147 melanoma cell lines, which were

1145 challenged with either Ctrl or MART-1-specific T cells for eight hours (outlined in Fig.

1146 4d). The gene set is based on genes that were relatively stronger induced in

1147 sgSTUB1-expressing cells after MART-1-specific T cell challenge.

1148

1149

1150

1151

1152

1153

1154 


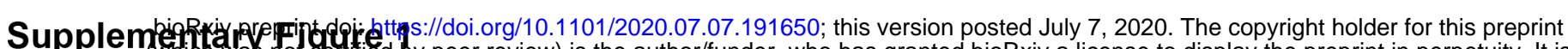

a

C

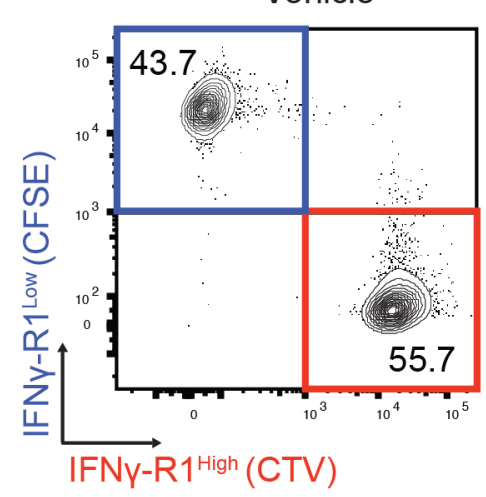

+ Vehicle made available under 99 F PYY-NC-ND 4.0 International license.
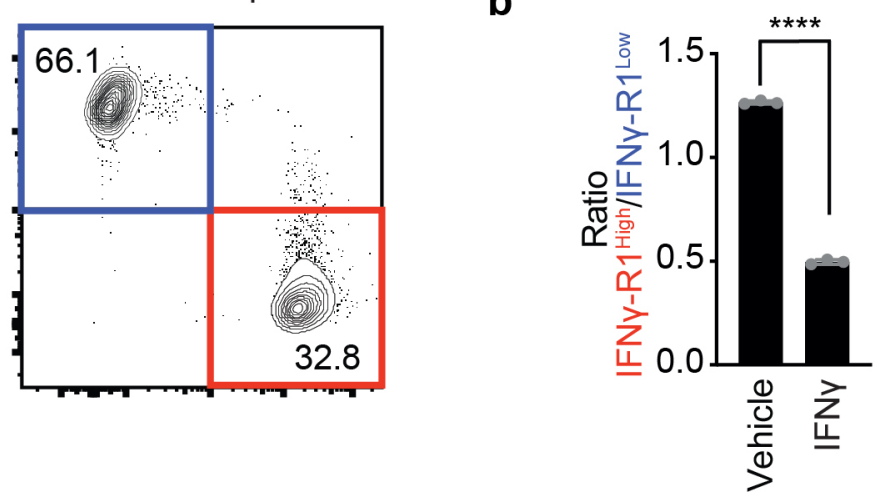
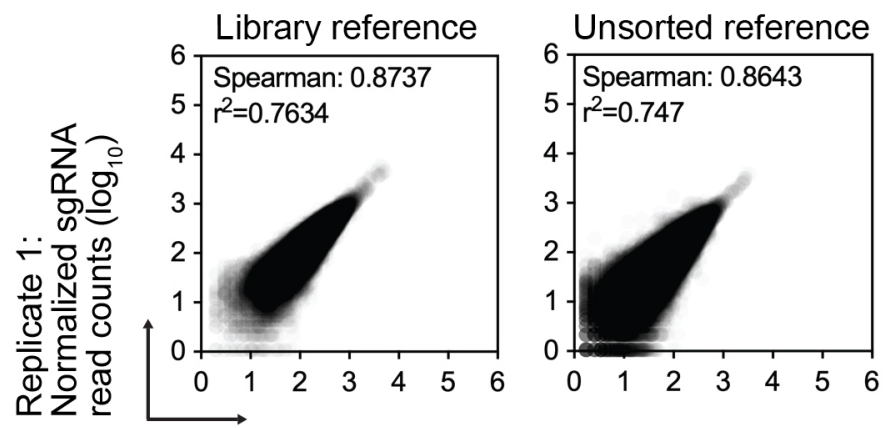

Replicate 2:

Normalized sgRNA

read counts $\left(\log _{10}\right)$

d
D10

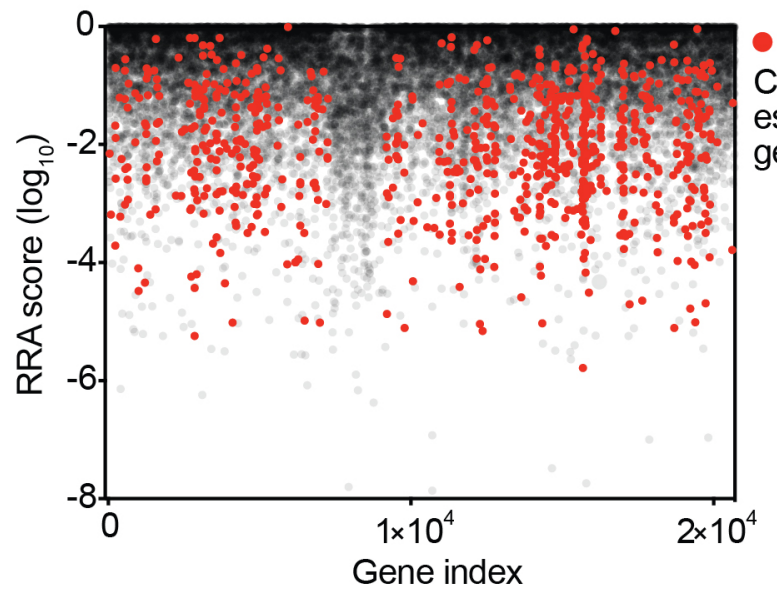

e

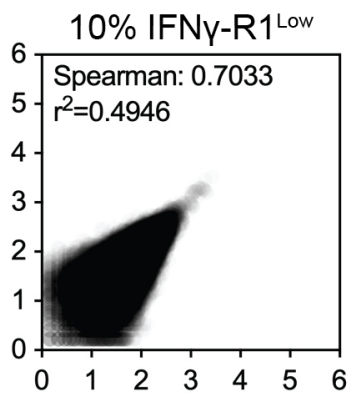

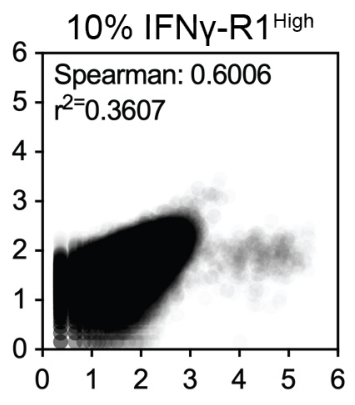

SK-MEL-23

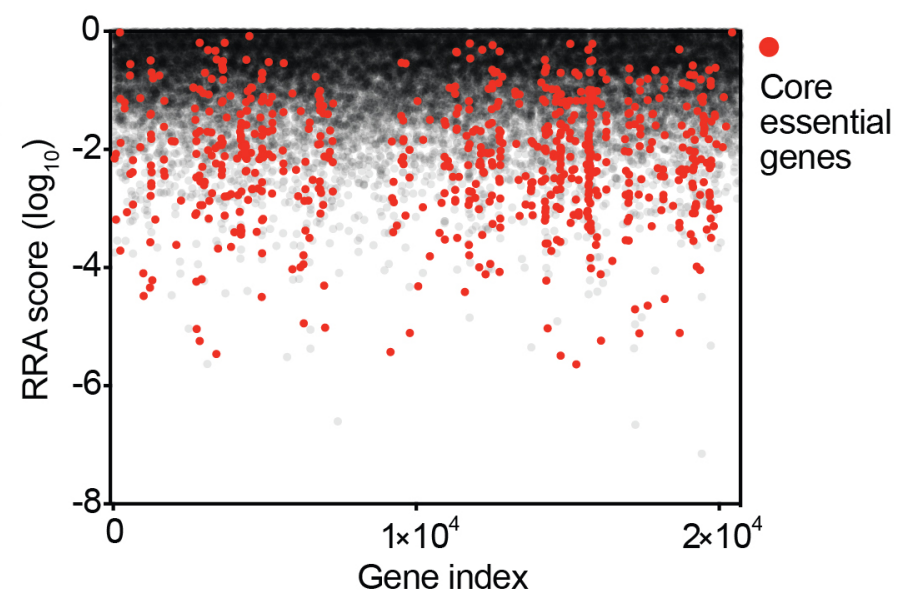

f

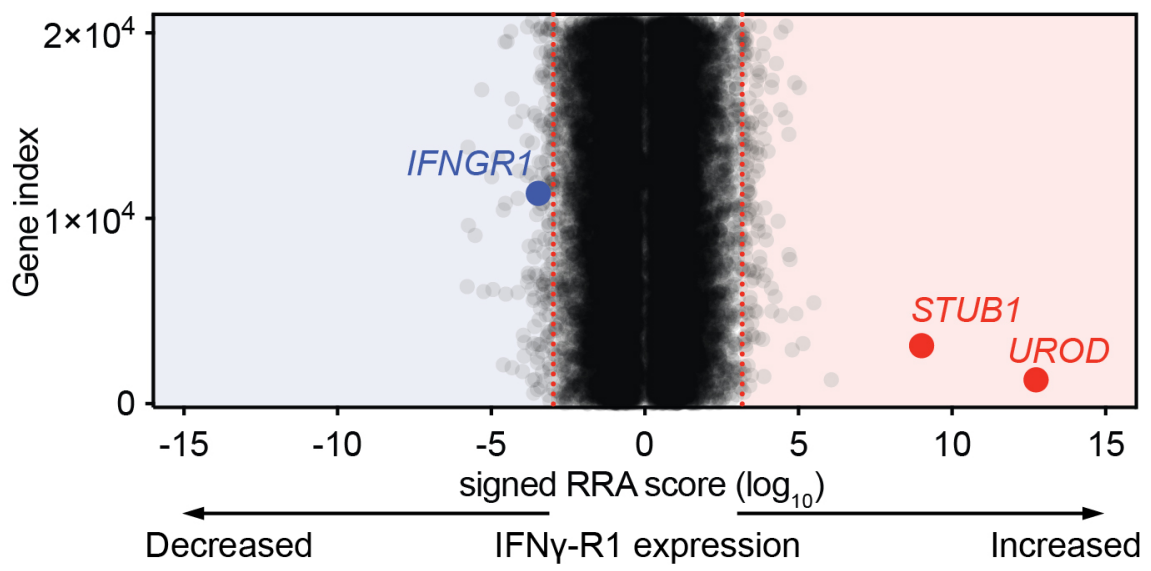




\section{Supplementary Figure Legends}

1157 Supplementary Figure 1: Genome-wide CRISPR/Cas9 knockout screen

1158 identifies negative regulators of IFNy-R1 expression to modulate its cell surface

1159 abundance.

1160 a, Flow cytometry plot of in vitro competition assay of IFNy-R1 ${ }^{\text {High }}$ vs. IFNy-R1 ${ }^{\text {Low }}$ cells

1161 treated with either vehicle or $25 \mathrm{ng} / \mathrm{ml} \mathrm{IFNy}$ for five days.

1162 b, Quantification of the ratio IFNy-R1 ${ }^{\text {High }}$ : IFNY-R1 $1^{\text {Low }}$ in competition assay of (a).

1163 c, Correlation plots of $\log _{10}$-transformed normalized read counts of sgRNAs in

1164 genome-wide CRISPR-KO screen in D10 melanoma cell line between replicates.

1165 d, e, $\log _{10}$-transformed RRA scores of depleted genes comparing library reference

1166 sample to unsorted bulk population in D10 (d) and SK-MEL-23 cells (e). Highlighted

1167 in red: core essential genes. y-axis: RRA score, $x$-axis: gene index.

1168 f, Results of screen outlined in (Figure 1f) for SK-MEL-23 cells. x-axis: signed $\log _{10^{-}}$

1169 transformed signed MAGeCK robust rank aggregation (RRA) score for each gene; $y$ -

1170 axis: gene index. Red dotted lines indicate FDR cutoff $<0.25$ for genes enriched in

$117110 \%$ of cells with the highest (right) or lowest (left) IFNGR1 expression.

1172 Mean $\pm S D$ in $(\mathbf{b}),{ }^{* * * *} p<0.0001$, unpaired t-test for three biological replicates. 
Supplemenftanye Frigdpretens://doi.org/10.1101/2020.07.07.191650; this version posted July 7, 2020. The copyright holder for this preprint which was not certified by peer review) is the author/funder, who has granted bioRxiv a license to display the preprint in perpetuity. It is

a $\quad$ sgCtrl

sgSTUB1

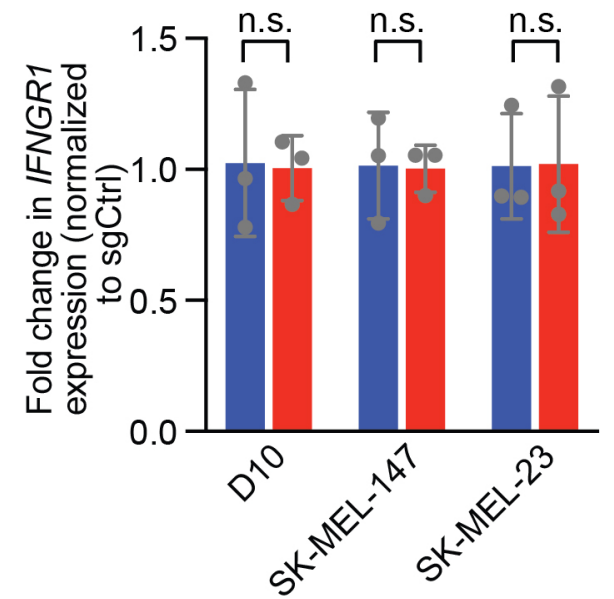

d b

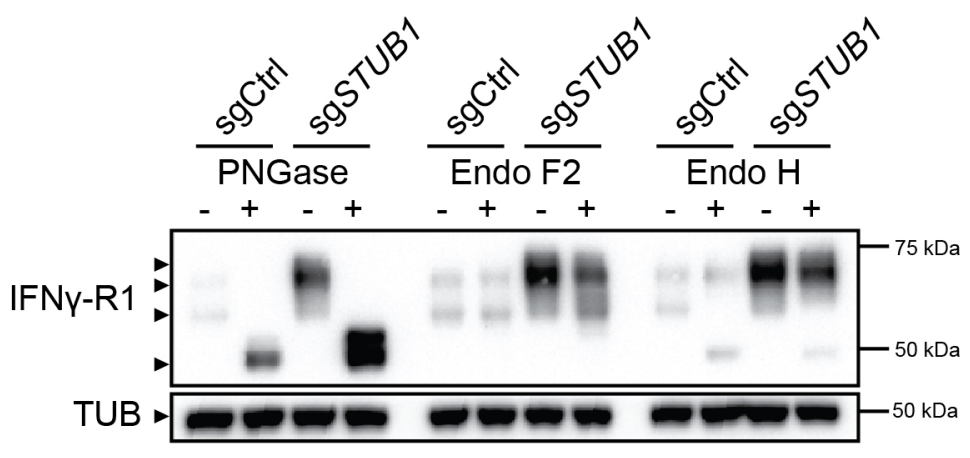

C

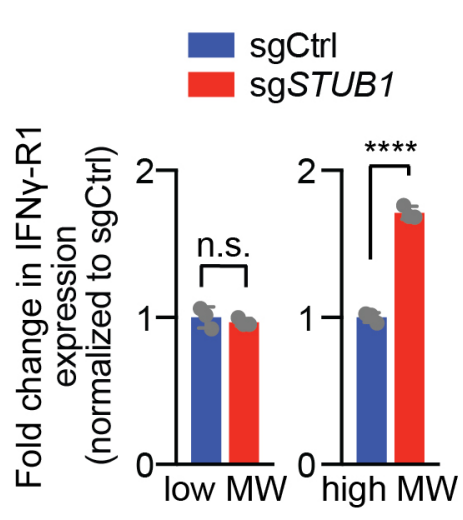

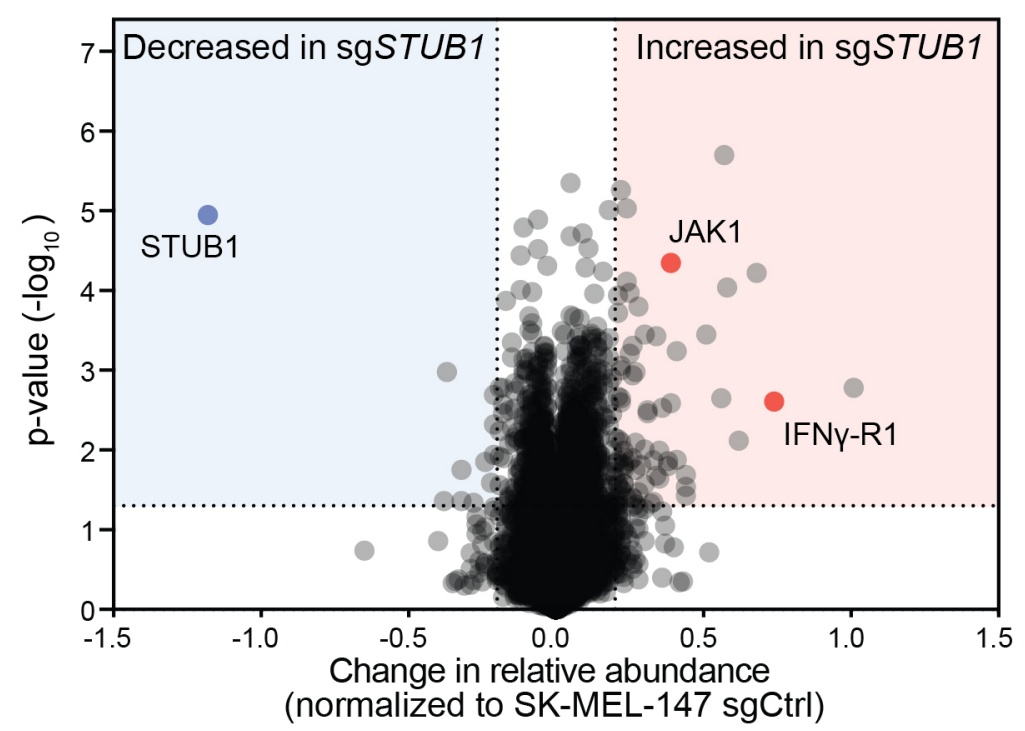

h

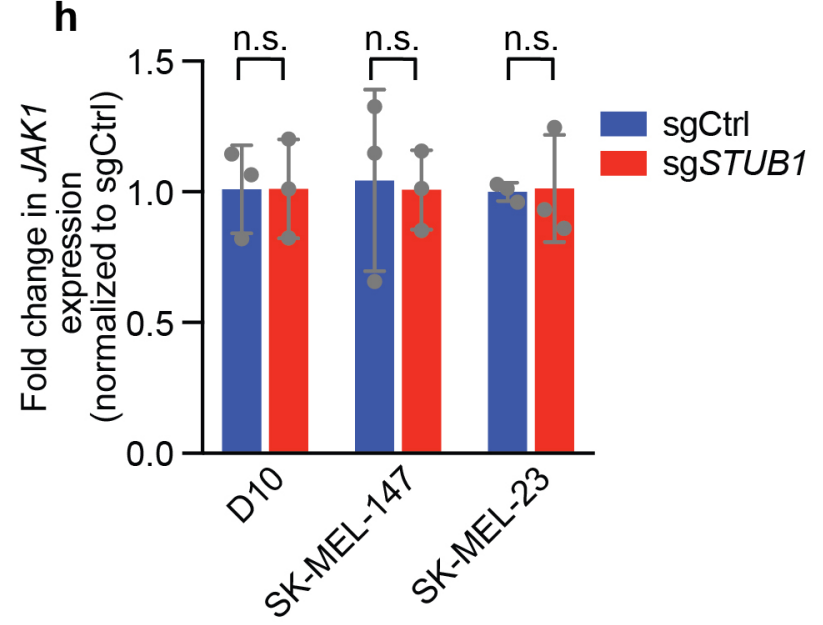

i

SK-MEL-23

sgSTUB

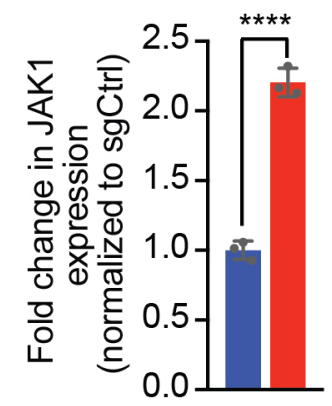

BLM

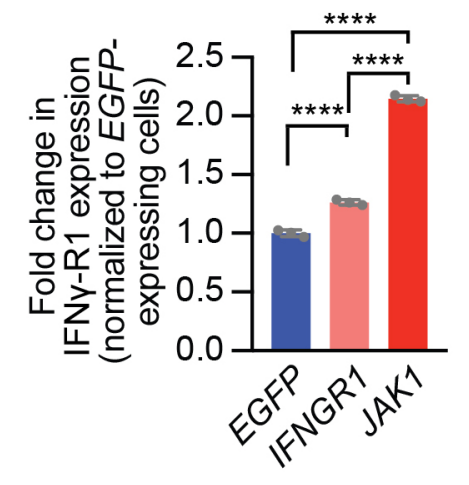

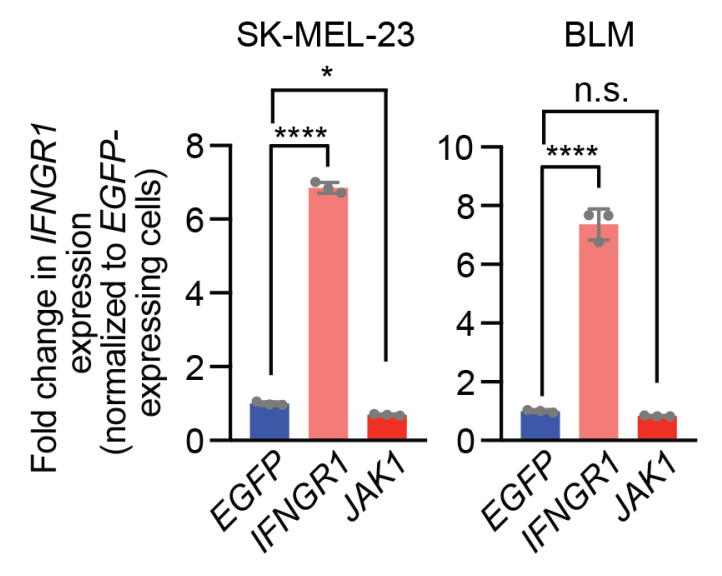


1175 Supplementary Figure 2: STUB1 destabilizes cell surface IFNY-R1 in JAK11176 dependent and JAK1-independent manners.

1177 a, Results of qPCR analysis for IFNGR1 mRNA expression (relative to RPL13 1178 expression) in D10, SK-MEL-147 and SK-MEL-23 cells expressing either sgCtrl or 1179 sgSTUB1.

1180 b, Immunoblot of whole cell lysates treated with the indicated deglycosylating 1181 enzymes. Whole cell lysates were collected from D10 melanoma cells expressing 1182 either sgCtrl or sgSTUB1. Whole cell lysates were immunoblotted for IFNy-R1 and 1183 Tubulin.

1184 c, Quantification of low and high molecular weight IFNy-R1 protein levels (relative to 1185 loading control) in D10 melanoma cells from immunoblot shown in (Figure 2d).

1186 d, Immunoblot of immuno-precipitated cell surface proteins using biotin labelling in 1187 D10 melanoma clone deficient in IFNGR1, or D10 melanoma cell pool expressing 1188 either sgCtrl or sgSTUB1. Following immunoprecipitation of biotin-labelled proteins, 1189 samples were immunoblotted for IFNy-R1. The right-most lane represents $10 \%$ of the 1190 whole cell lysate of sgSTUB1-expressing cells.

1191 e, Results of proteomic profiling of SK-MEL-147 melanoma cells expressing either 1192 sgCtrl or sgSTUB1. Highlighted are the top differentially regulated proteins shared 1193 between sgCtrl and sgSTUB1-expressing D10 and SK-MEL-147 cells (Fig. 2c).

1194 f, Quantification of IFNy-R1 protein levels (relative to loading control) in D10 1195 melanoma cells from immunoblot shown in (Figure 2d).

1196 g, Quantification of JAK1 protein levels (relative to loading control) in D10 melanoma 1197 cells from immunoblot shown in (Figure 2d).

$1198 \mathbf{h}$, Results of qPCR analysis for JAK1 mRNA expression (relative to RPL13 1199 expression) in D10, SK-MEL-147 and SK-MEL-23 cells expressing either sgCtrl or 1200 sgSTUB1.

1201 i, Quantification of IFNY-R1 expression (relative to EGFP-ORF-expressing cells) by 1202 flow cytometry in SK-MEL-23 and BLM-M melanoma cells expressing EGFP-ORF, 1203 IFNGR1-ORF and JAK1-ORF.

$1204 \mathrm{j}$, Results of qPCR analysis for IFNGR1 mRNA expression (relative to RPL13 1205 expression) in SK-MEL-23 and BLM-M melanoma cells expressing EGFP-ORF, 1206 IFNGR1-ORF and JAK1-ORF. Relative IFNGR1 expression was normalized to EGFP1207 ORF-expressing cells. 
1208 Mean \pm SD in (a), D10: n.s. $p=0.918$, SK-MEL-147: n.s. $p=0.933$, SK-MEL-23: n.s. $1209 \mathrm{p}=0.968$, unpaired t-tests were performed for each cell line, each three biological 1210 replicates.

1211 Mean $\pm S D$ in $(\mathbf{c}),{ }^{* * * *} p<0.0001$, n.s. $p=0.5029$, unpaired t-test for three biological 1212 replicates.

1213 Mean $\pm S D$ in $(\mathbf{f}),{ }^{* * *} p=0.0002$, unpaired t-test for three biological replicates.

1214 Mean $\pm S D$ in $(\mathbf{g}),{ }^{* * * *} p<0.0001$, unpaired t-test for three biological replicates.

1215 Mean \pm SD in (h), D10: n.s. $p=0.99$, SK-MEL-147: n.s. $p=0.877$, SK-MEL-23: n.s. $1216 p=0.921$, multiple t-test for three biological replicates.

1217 Mean $\pm S D$ in $(\mathbf{i}),{ }^{* *} p=0.0093,{ }^{* * *} p<0.0001$, ordinary one-way ANOVA for three 1218 biological replicates with Tukey post hoc testing.

1219 Mean \pm SD in (j), ${ }^{*} p=0.0103,{ }^{* * * *} p<0.0001$, n.s. $p=0.7409$, ordinary one-way ANOVA 1220 for three biological replicates with Dunnett post hoc testing. 
SupplempientamypFigeirtetp3:/doi.org/10.1101/2020.07.07.191650; this version posted July 7, 2020. The copyright holder for this preprint
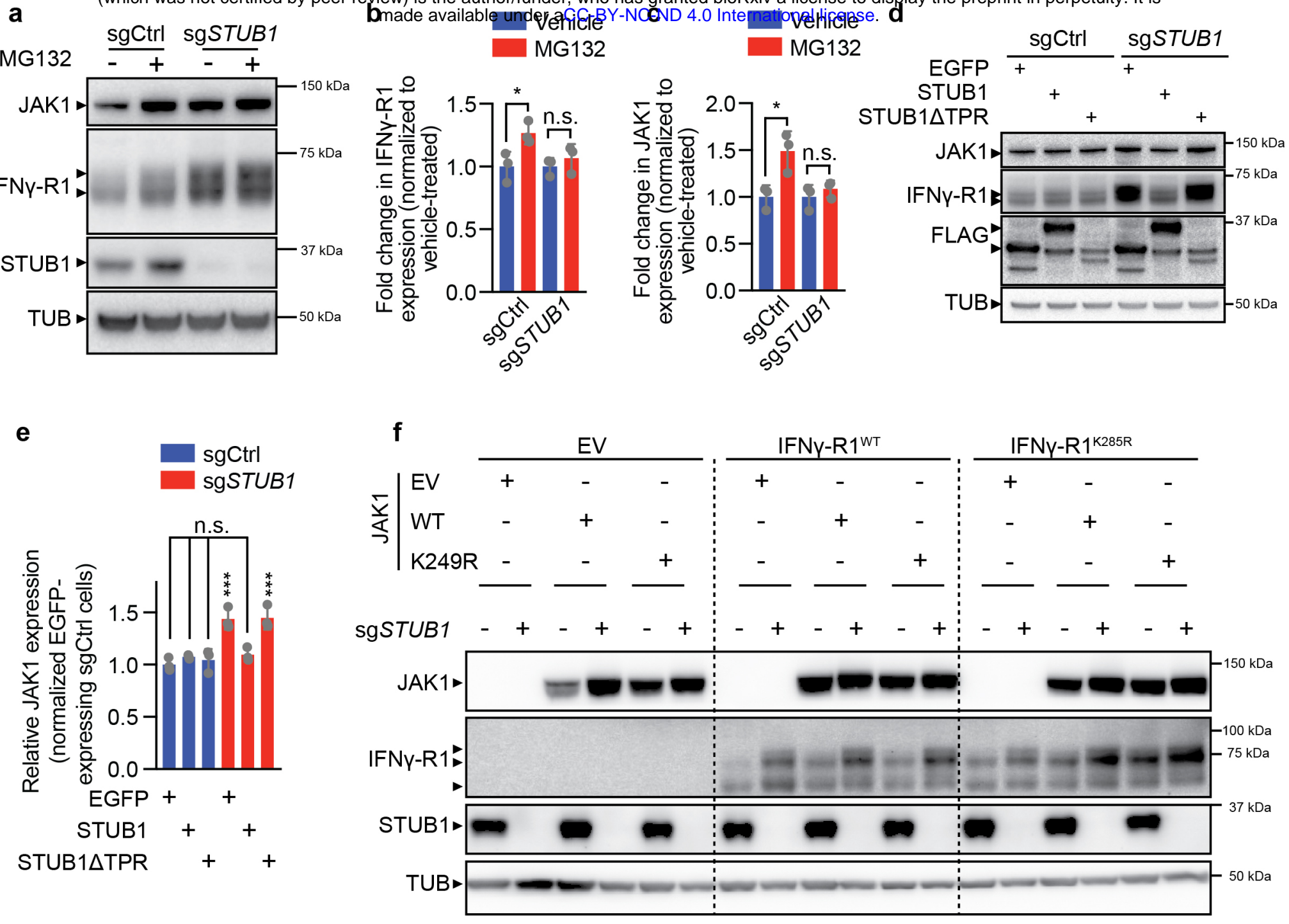

g

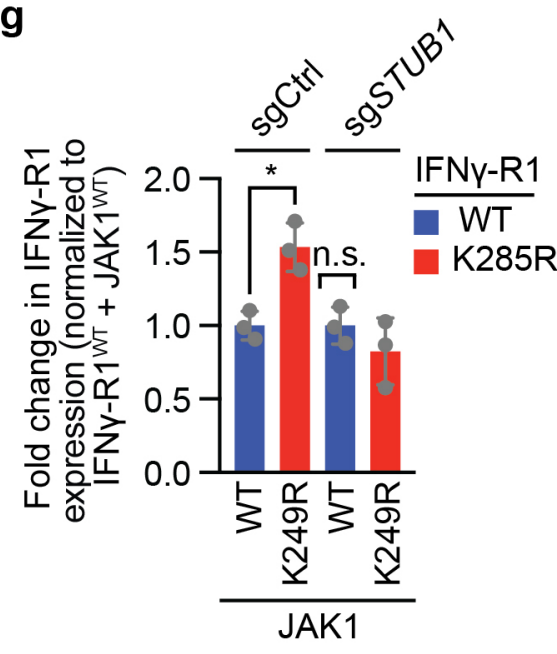

h

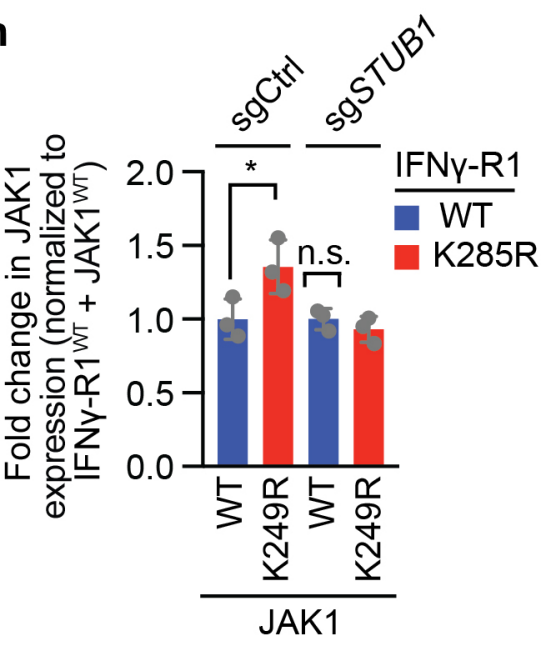

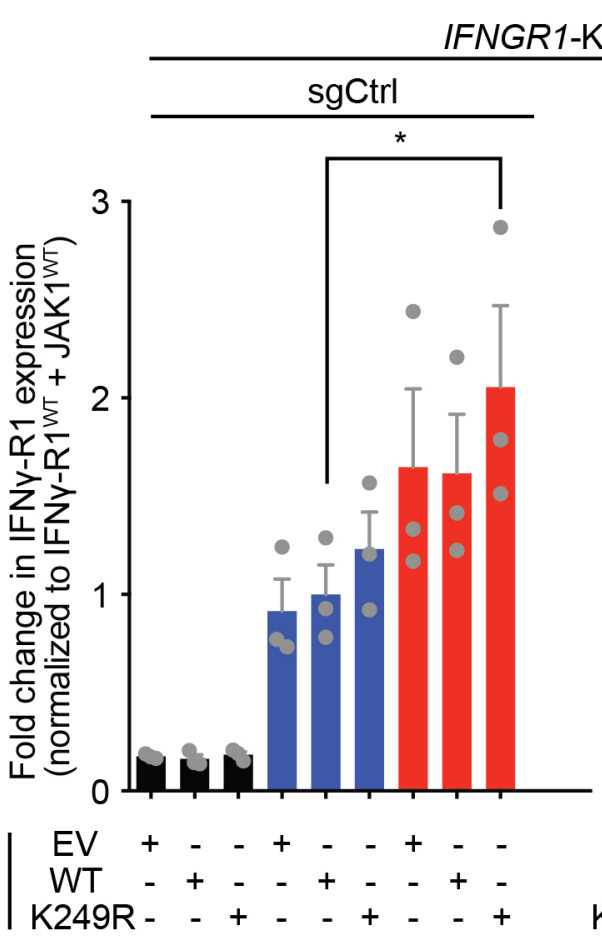

IFNGR1-KO +JAK1-KO

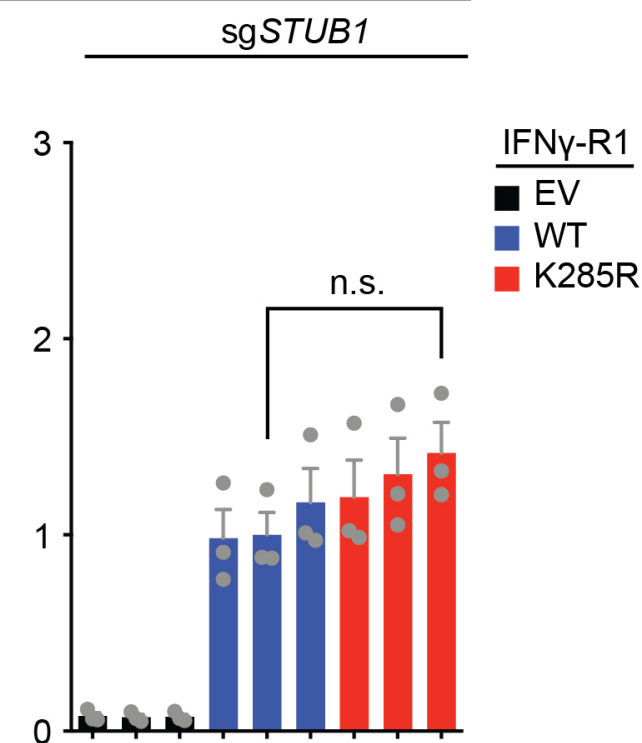

EV + - - + - - + - -

K249R- - + - - + - + 
1222 Supplementary Figure 3: STUB1 drives proteasomal degradation of IFNy receptor complex through IFNY-R1 ${ }^{\mathrm{K} 285}$ and JAK1 ${ }^{\mathrm{K} 249}$ residues.

1224 a, Immunoblot of SK-MEL-147 melanoma cells expressing either sgCtrl or sgSTUB1

1225 treated with either vehicle or $10 \mu \mathrm{M}$ MG132 for four hours. Whole-cell lysates were 1226 immunoblotted for the indicated proteins (TUB is Tubulin).

1227 b, Quantification of IFNy-R1 protein levels (relative to loading control and normalized 1228 to vehicle-treated group) from (a).

1229 c, Quantification of JAK1 protein levels (relative to loading control and normalized to 1230 vehicle-treated group) from (a).

1231 d, Immunoblot of D10 melanoma cells expressing either sgCtrl or sgSTUB1, that 1232 ectopically express either 3xFLAG-tagged EGFP, full length STUB1 or STUB1 lacking 1233 N-terminal residues 1-72 of the TPR domain. Whole cell lysates were blotted for the 1234 indicated proteins (TUB is Tubulin).

1235 e, Quantification of JAK1 protein levels (relative to loading control and normalized to 1236 EGFP- and sgCtrl-expressing cells) from immunoblot depicted in (d).

1237 f, Immunoblot of whole cell lysates from IFNGR1-KO + JAK1-KO D10 melanoma 1238 clones reconstituted with the indicated IFNGR1 and JAK1 cDNAs, for the indicated 1239 proteins (TUB is Tubulin).

1240 g, Quantification of IFNy-R1 protein levels on immunoblot in Figure 3j (relative to 1241 loading control and normalized to IFNY-R1 ${ }^{\mathrm{WT}}$ and JAK1 ${ }^{\mathrm{WT}}$-expressing cells) in 1242 IFNGR1-KO + JAK1-KO D10 melanoma clones expressing either IFNy-R1 ${ }^{\mathrm{WT}}$ and 1243 JAK1 ${ }^{\mathrm{WT}}$ or with IFNY-R1 ${ }^{\mathrm{K} 285 \mathrm{R}}$ and JAK1 ${ }^{\mathrm{K} 249 \mathrm{R}}$.

$1244 \mathbf{h}$, Quantification of JAK1 protein levels on immunoblot in Figure 3j (relative to loading 1245 control and normalized to IFNY-R1 ${ }^{\mathrm{WT}}$ and JAK1 ${ }^{\mathrm{WT}}$-expressing cells) in IFNGR1-KO + 1246 JAK1-KO D10 melanoma clones expressing either IFNY-R1 ${ }^{\mathrm{WT}}$ and JAK1 ${ }^{\mathrm{WT}}$ or with 1247 IFNy-R1 ${ }^{\mathrm{K} 285 \mathrm{R}}$ and JAK1 ${ }^{\mathrm{K} 249 \mathrm{R}}$.

1248 i, Quantification of IFNY-R1 expression by flow cytometry in IFNGR1-KO + JAK1-KO 1249 D10 melanoma clones reconstituted with the indicated IFNGR1 and JAK1 cDNAs 1250 (outlined in Figure 3f), shown as fold-change of IFNY-R1 MFI relative to IFNy-R1WT + 1251 JAK1 ${ }^{\mathrm{WT}}$-expressing cells for each respective genotype. EV = empty vector control.

1252 Mean $\pm S D$ in $(\mathbf{b}),{ }^{*} p=0.0435$, n.s. $p=0.8357$, ordinary one-way ANOVA for three 1253 biological replicates with Tukey post hoc testing.

1254 Mean $\pm S D$ in $(\mathbf{c}),{ }^{*} p=0.0138$, n.s. $p=0.8846$, ordinary one-way ANOVA for three 1255 biological replicates with Tukey post hoc testing. 
1256 Mean $\pm S D$ in $(\mathbf{e}),{ }^{* * *} p=0.004,{ }^{* * *} p=0.003$, n.s. $p=0.7405, p=9996, p=0.972$, ordinary

1257 one-way ANOVA for three biological replicates with Tukey post hoc testing.

1258 Mean \pm SD in $(\mathbf{g}),{ }^{*} p=0.0156$, n.s. $p=0.5704$, ordinary one-way ANOVA for three

1259 immunoblots with Tukey post hoc testing.

1260 Mean $\pm S D$ in $(\mathbf{h}),{ }^{*} p=0.0366$, n.s. $p=0.9068$, ordinary one-way ANOVA for three 1261 biological replicates with Tukey post hoc testing.

1262 Mean $\pm S D$ in $(\mathbf{i}),{ }^{*} p=0.036$, n.s. $p=0.9812$, ordinary one-way ANOVA for three

1263 biological replicates, with Tukey post hoc testing. 
Supplementamyp Figigunerd://doi.org/10.1101/2020.07.07.191650; this version posted July 7, 2020. The copyright holder for this preprint (which was not certified by peer review) is the author/funder, who has granted bioRxiv a license to display the preprint in perpetuity. It is

a

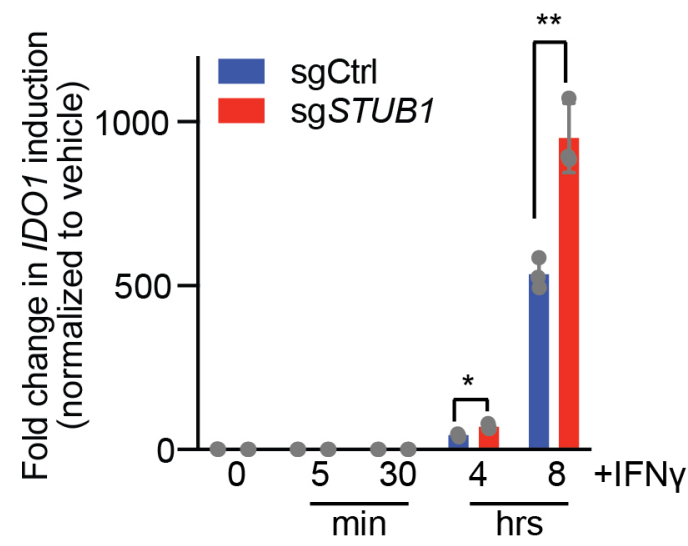

d

D10 \& SK-MEL-147

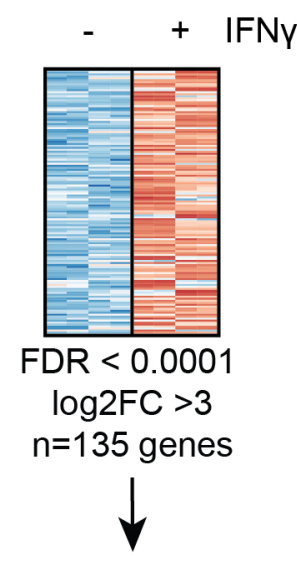

IFNy response genes b

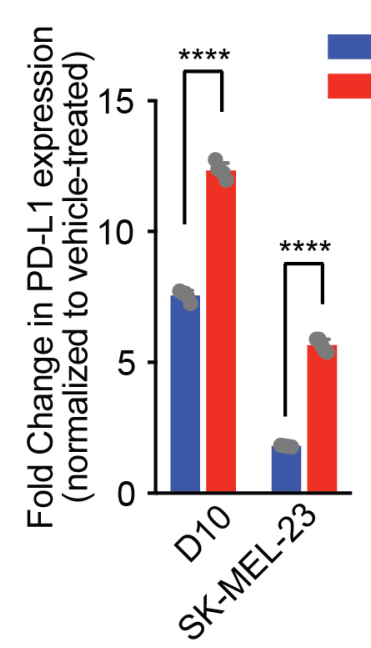

e

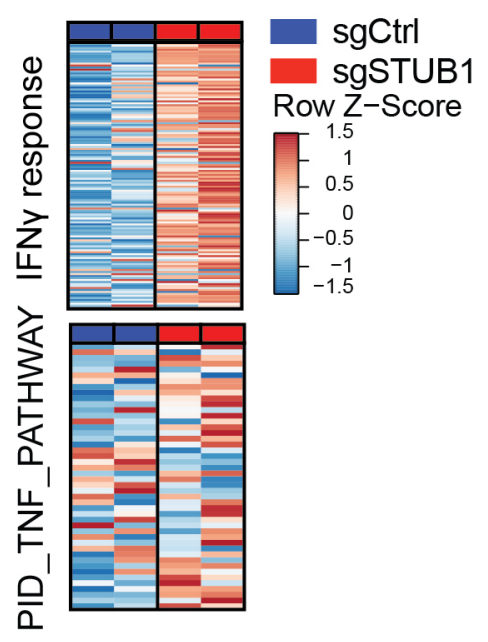

sgCtrl

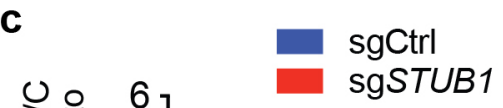

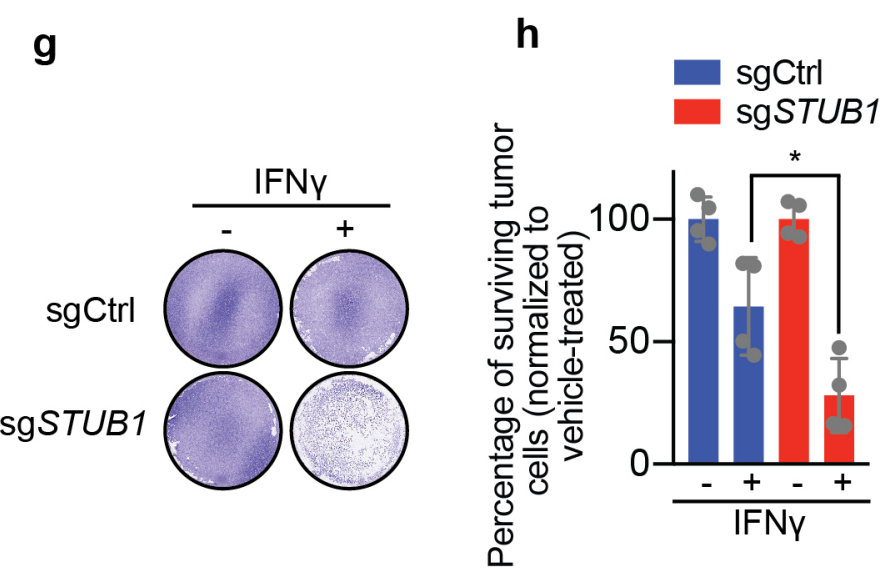

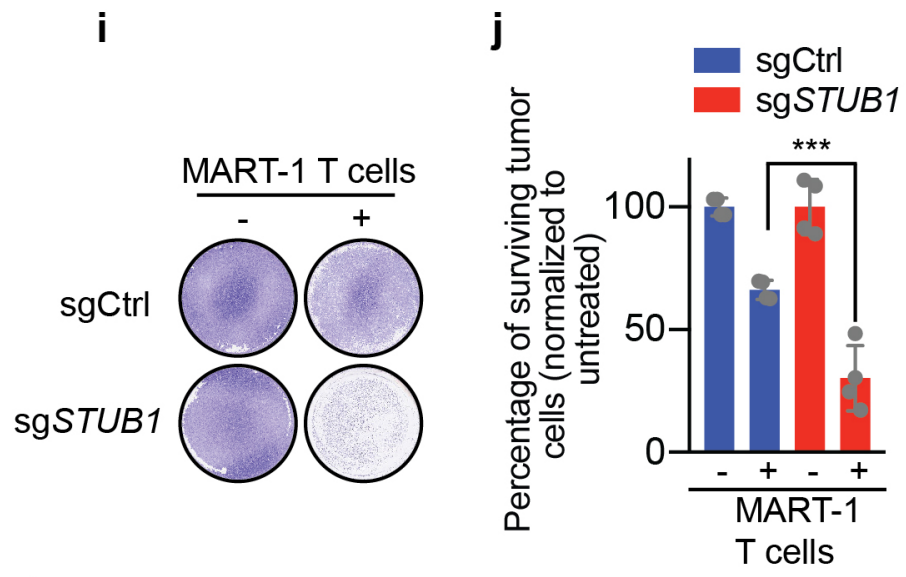

\section{f}

Increased

$\square$ Decreased $p=8.001 e-13$
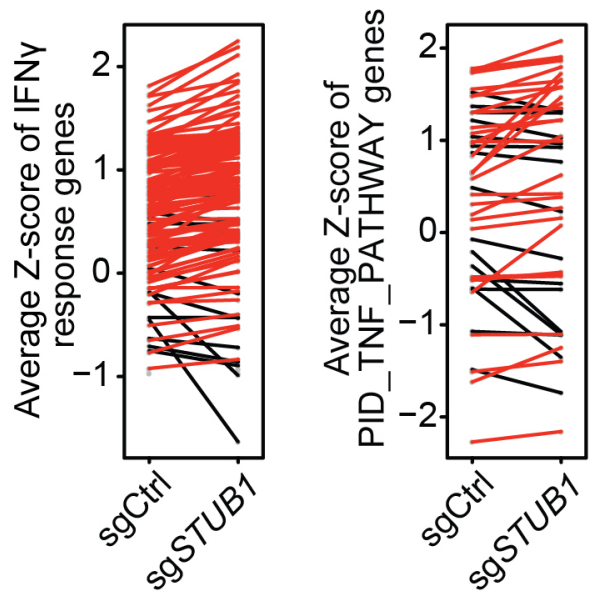

I
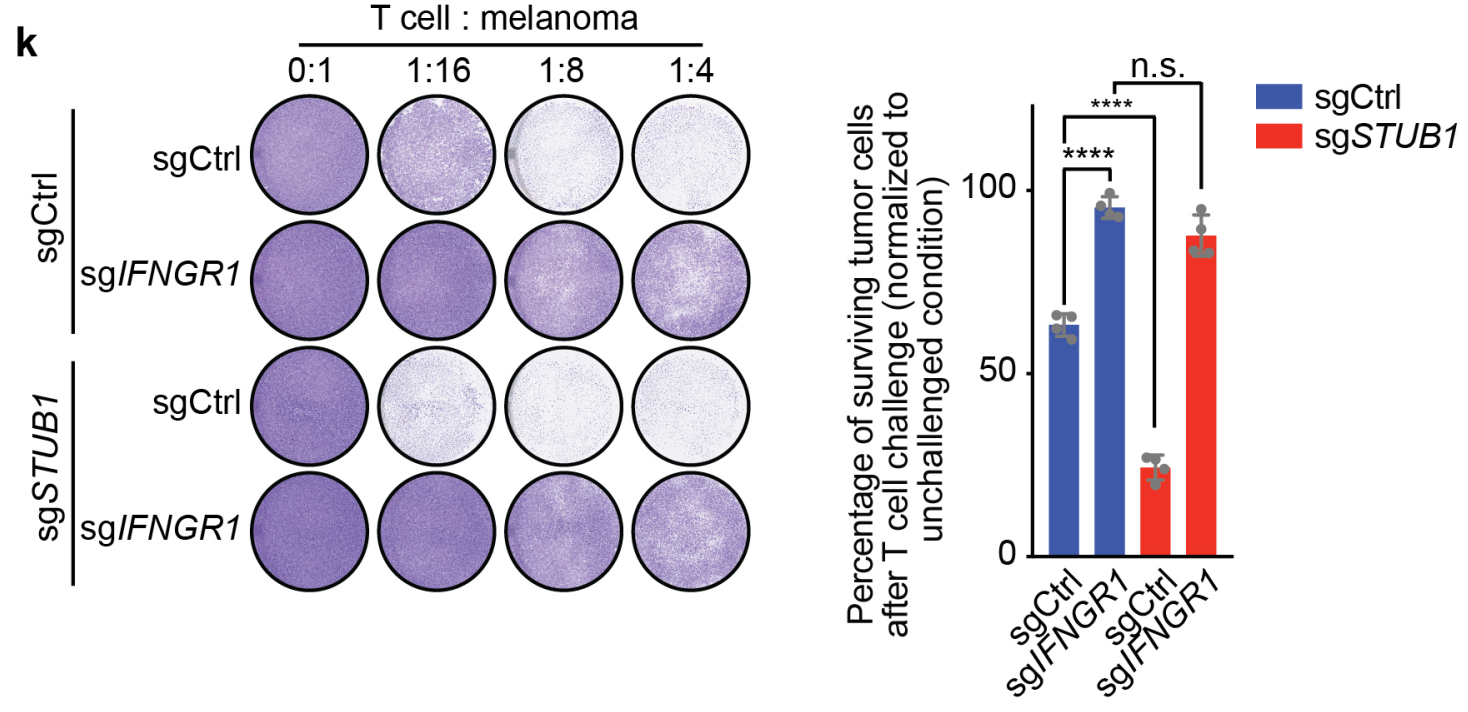
1266 Supplementary Figure 4: STUB1 inactivation sensitizes melanoma cells to 1267 cytotoxic T cells through amplified IFNy signaling.

1268 a, qPCR analysis for IDO1 mRNA expression in D10 melanoma cells expressing either $1269 \mathrm{sgCtrl}$ or sgSTUB1, which were treated with $25 \mathrm{ng} / \mathrm{ml}$ IFNy for the indicated duration.

1270 b, Flow cytometry analysis of IFNY-induced PD-L1 expression on cells expressing 1271 either sgCtrl or sgSTUB1 after 24 hours treatment with $5 \mathrm{ng} / \mathrm{ml} \mathrm{IFNy}$ for D10 cells and $12720.5 \mathrm{ng} / \mathrm{ml}$ IFNy for SK-MEL-23 cells.

1273 c, Flow cytometry analysis of IFNY-induced HLA-A/B/C expression on SK-MEL-23 1274 melanoma cells expressing either sgCtrl or sgSTUB1 after 24 hours treatment with 0.5 $1275 \mathrm{ng} / \mathrm{ml}$ IFNy for SK-MEL-23.

1276 d, Differential gene expression of D10 and SK-MEL-147 melanoma cells lines after 1277 treatment with IFNy for eight hours was used to derive an IFNy response gene set.

$1278 \mathbf{e}$, Differential gene expression analysis of IFNy response genes (derived by treating 1279 D10 and SK-MEL-147 melanoma cells with IFNy for eight hours, depicted in d) and 1280 PID_TNF_PATHWAY genes in SK-MEL-147 melanoma cells co-cultured with MART$12811 \mathrm{~T}$ cells for eight hours.

$1282 \mathbf{f}$, Difference in either IFNy response gene expression or expression of 1283 PID_TNF_PATHWAY genes between sgCtrl and sgSTUB1-expressing SK-MEL-147 1284 melanoma cells following MART-1 T cell challenge for eight hours.

1285 g, Colony formation assay of SK-MEL-147 melanoma cells expressing sgCtrl or 1286 sgSTUB1 treated with either vehicle or $50 \mathrm{ng} / \mathrm{ml}$ IFNy for five days.

$1287 \mathbf{h}$, Quantification of colony formation assay shown in $(\mathbf{g})$.

1288 i, Colony formation assay of SK-MEL-147 melanoma cells expressing sgCtrl or 1289 sgSTUB1 treated with either no or MART-1 T cells for 24 hours and subsequent culture 1290 for four days.

1291 j, Quantification of colony formation assay shown in (i).

1292 k, Colony formation assay of SK-MEL-147 melanoma cells expressing the indicated 1293 sgRNAs that were co-cultured with either no T cell or MART-1 T cells at T cell : 1294 melanoma cell ratios 1:16, 1:8 and 1:4 (left to right) for 24 hours and subsequent 1295 culture for four days.

1296 I, Quantification of crystal violet stained colony formation assays from $(\mathbf{k})$ at a T cell : 1297 melanoma cell ratio of 1:16.

1298 Mean \pm SD in $(\mathbf{a}),{ }^{* *} p=0.0034,{ }^{*} p=0.012$, multiple t-tests for three biological replicates. 
1299 Mean \pm SD in (b), ${ }^{* * *} \mathrm{p}<0.0001$ for SK-MEL-23, ${ }^{* * * *} \mathrm{p}<0.0001$, unpaired t-test for five

1300 biological replicates.

1301 Mean \pm SD in $(\mathbf{c}),{ }^{* * * *} \mathrm{p}<0.0001$, unpaired t-test for five biological replicates.

1302 Average Z-score of respective genes in (g) from two biological replicates with paired 1303 t-test.

1304 Mean \pm SD in $(\mathbf{h}),{ }^{*} \mathrm{p}=0.0132$, ordinary one-way ANOVA for four biological replicates 1305 with Tukey post hoc testing.

1306 Mean \pm SD in $(\mathbf{j}),{ }^{* * *} \mathrm{p}=0.0006$, ordinary one-way ANOVA for four biological replicates 1307 with Tukey post hoc testing.

1308 Mean $\pm S D$ in (I), n.s. $p=0.0713,{ }^{* * * *} p<0.0001$, ordinary one-way ANOVA for four 1309 biological replicates with Tukey post hoc testing. 


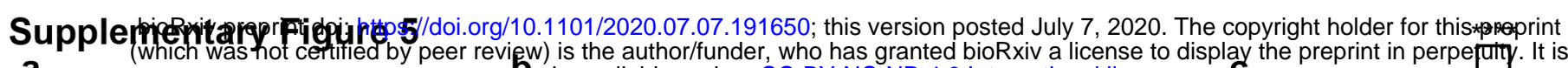

a ade available under aCC-BY-NC-ND 4.0 International license.

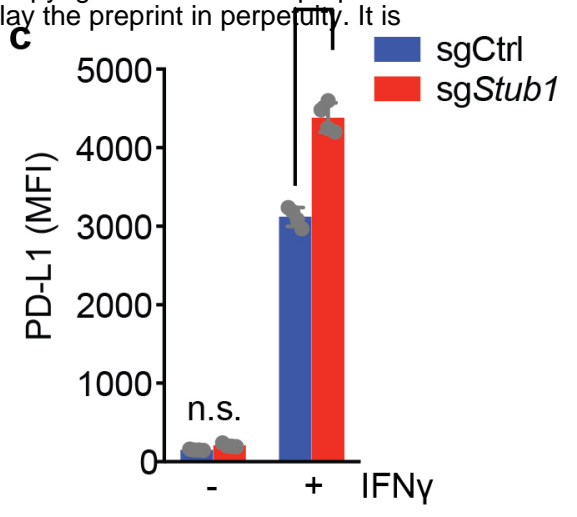

d

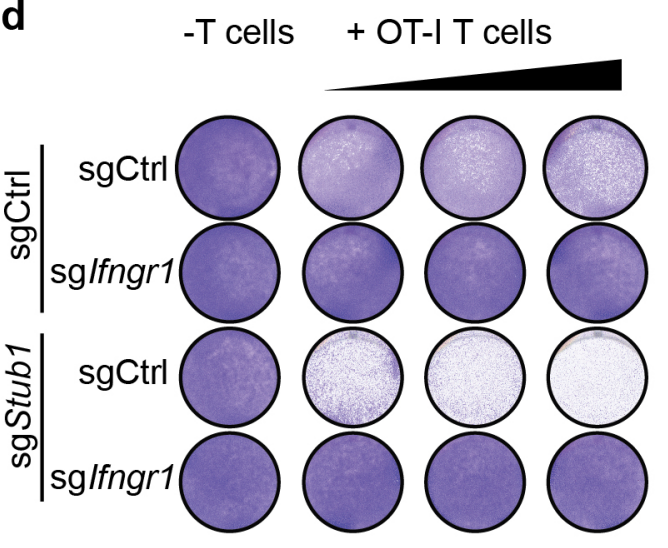

f

STUB1-KO signature expression

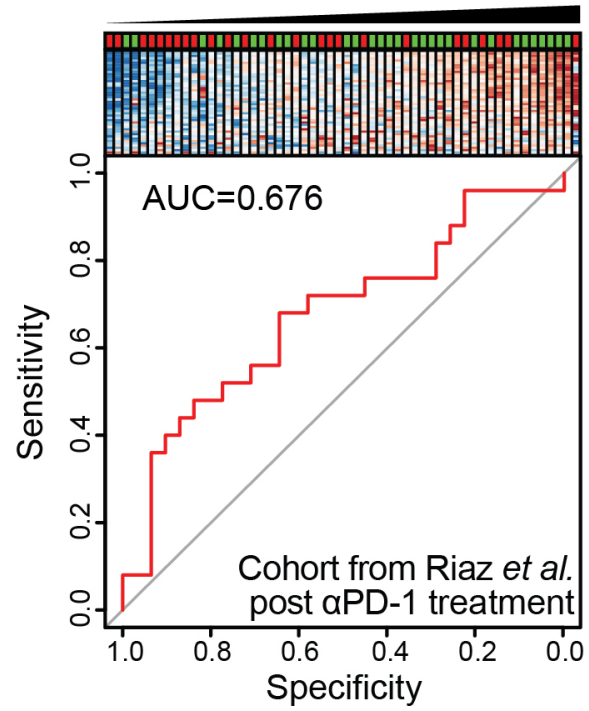

i

STUB1-KO-specific genes only

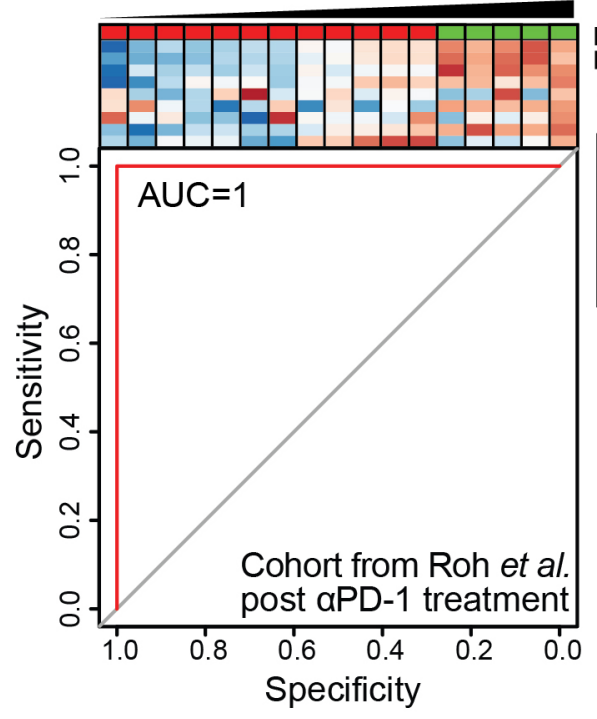

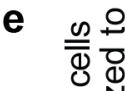

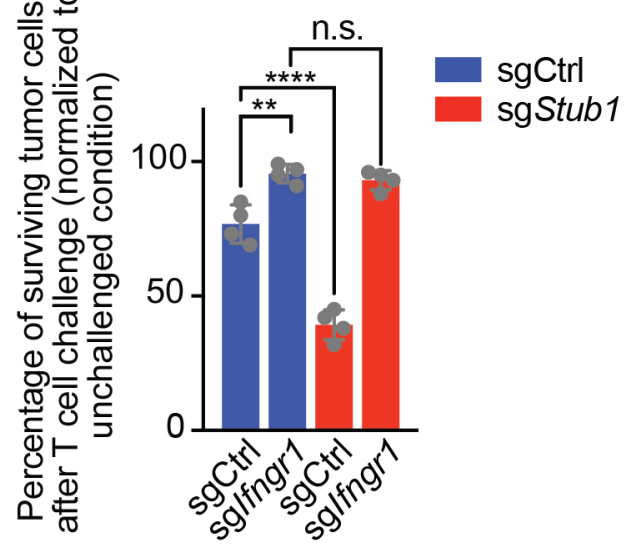

g

h
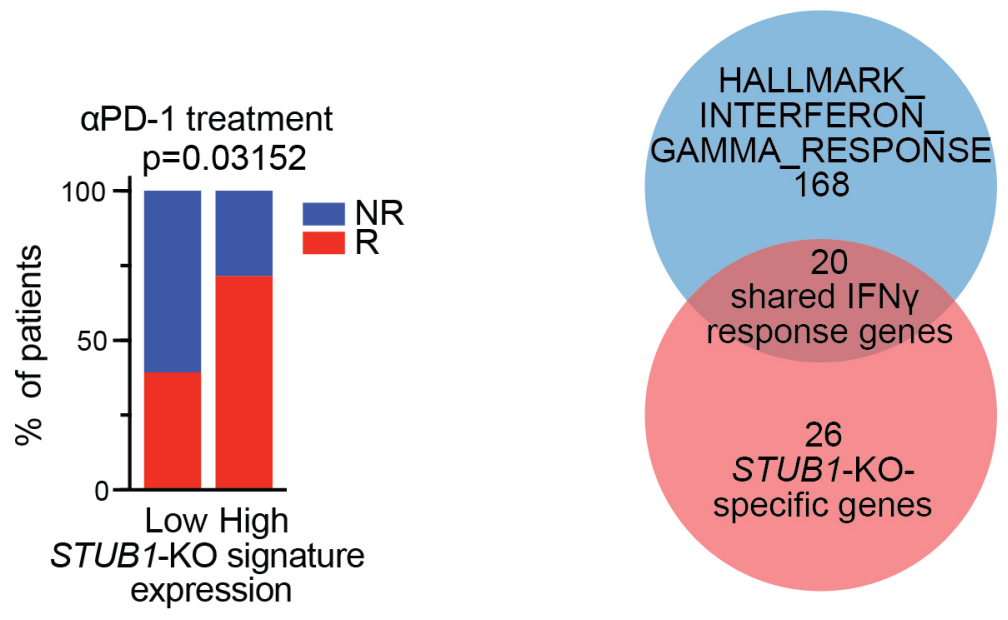

j

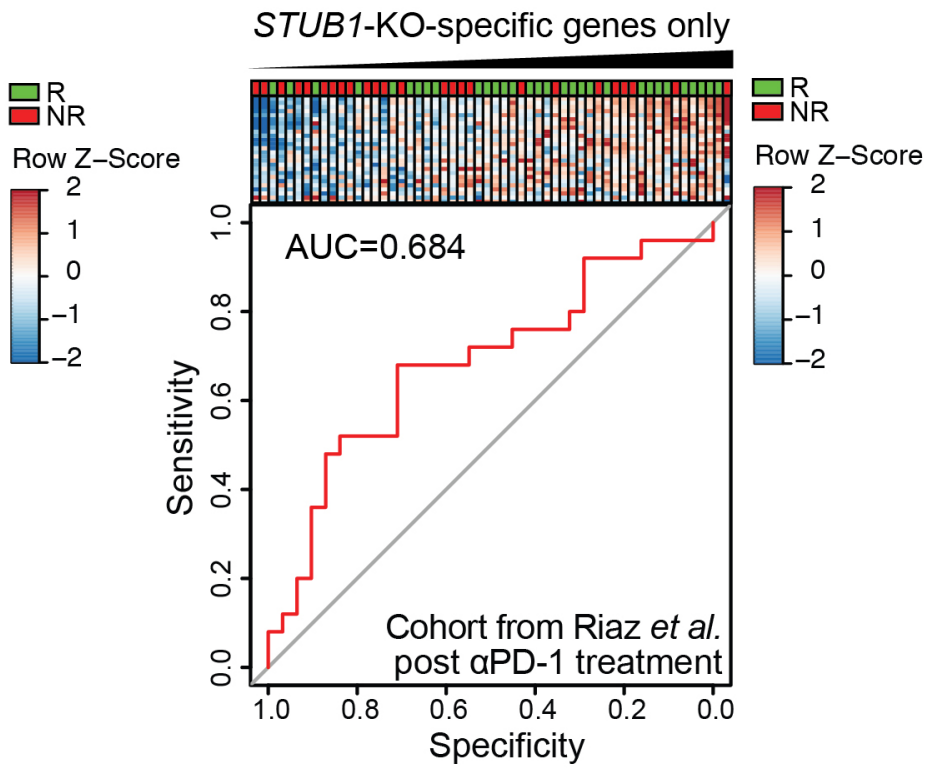


1312 Supplementary Figure 5: STUB1 inactivation and anti-PD-1 treatment constitute

1313 a rational combination therapy approach.

1314 a, Immunoblot of murine melanoma cell lines expressing either sgCtrl or sgStub1.

1315 Whole cell lysates were blotted for the indicated proteins (TUB is Tubulin).

1316 b, Flow cytometry histograms showing Ifngr1 expression in indicated murine

1317 melanoma cell lines expressing either sgCtrl (blue) or sgStub1 (red). FMO (grey) =

1318 Fluorescence minus one, PE=Phycoerythrin.

1319 c, Flow cytometry analysis of IFNy-induced PD-L1 expression in B16F10-dOVA cells 1320 expressing either sgCtrl or sgStub1. Cells were treated with $12 \mathrm{ng} / \mathrm{ml}$ murine IFNy for 132124 hours.

1322 d, Colony formation assay of B16F10-dOVA melanoma cells expressing the indicated 1323 sgRNAs and co-cultured with either no T cells or OT-I T cells at T cell : melanoma cell 1324 ratios $1: 1,2: 1$ and $4: 1$ (left to right).

1325 e, Quantification from (d) at a T cell : melanoma cell ratio of 4:1.

$1326 \mathbf{f}$, Same analysis as in Figure $\mathbf{5 g}$, for melanoma patients from the post aPD-11327 treatment cohort of Riaz et al. 2017.

$1328 \mathbf{g}$, Same analysis as in Figure 5h, for the post aPD-1-treatment cohort of Riaz et al. $1329 \quad 2017$

$1330 \mathbf{h}$, Venn diagram depicting the overlap between the 1331 HALLMARK_INTERFERON_GAMMA_RESPONSE gene set and the STUB1-KO 1332 signature gene set.

$1333 \mathbf{i}$, Same analysis as in Figure $\mathbf{5 g}$, for the post aPD-1-treatment cohort of Roh et al. 13342017 using the expression of the 26 genes specific to the STUB1-KO signature 1335 (outlined in $\mathbf{h}$ ).

$1336 \mathbf{j}$, Same analysis as in Figure 5g, for the post aPD-1-treatment cohort of Riaz et al. 13372017 using the expression of the 26 genes specific to the STUB1-KO signature 1338 (outlined in $\mathbf{h}$ ).

1339 Mean $\pm S D$ in $(\mathbf{c}),{ }^{* * * *} p p<0.0001$, n.s. $p=0.8893$, ordinary one-way ANOVA for four 1340 biological replicates with Tukey post hoc testing.

1341 Mean $\pm S D$ in $(\mathbf{e}),{ }^{* *} p=0.0012,{ }^{* * *} p<0.0001$, n.s. $p=0.9012$, ordinary one-way ANOVA 1342 for four biological replicates with Tukey post hoc testing. 